\title{
A STUDY OF EFFECTIVE LEADERSHIP IN THE CHINESE CONTEXT
}

Wai Kwan Lau, B.A., M.B.A.

Dissertation Prepared for the Degree of

DOCTOR OF PHILOSOPHY

UNIVERSITY OF NORTH TEXAS

August 2012

APPROVED:

Vicki L. Goodwin, Major Professor and Chair of the Department of Management

Lewis Taylor, Committee Member Mnjula Salimath, Committee Member Robert Pavur, Committee Member Mark A. Davis, Program Coordinator O. Finley Graves, Dean of College of Business Mark Wardell, Dean of the Toulouse Graduate School 
Lau, Wai Kwan. A Study of Effective Leadership in the Chinese Context. Doctor of Philosophy (Management), August 2012, 179 pages, 41 tables, 12 figures, 173 references, 77 titles.

Leadership has attracted a significant amount of scholarly attention in the past few decades. However, most research and theory contributions are to a great extent limited to accounting for leadership practices in the West (Littrell, 2002). This study is designed to develop an effective leadership model that works in the Chinese context. Paternalistic leadership, a dominant leadership style in an Eastern business environment, is compared with transformational leadership, a dominant leadership style in a Western business environment. The notion of transformational leadership was developed under the tutelage of Bernard Bass (1998). Transformational leadership is found to be compatible with collectivistic values (Walumbwa \& Lwwler, 2003) and is believed to be appealing and generalizable to Chinese leadership situations (Chen \& Farh, 1999). Other researchers have found that within Chinese organizations, leader behaviors are quite distinct from transformational leadership, referring to this leader style as paternalistic leadership (Redding, 1990; Cheng, 1995). The questions are asked, “Transformational or paternalistic leadership, which one is more effective in Chinese organizations? Is one type of leadership superior to the other one in the Chinese culture?” To answer these questions, a model is proposed to clarify the mediating effects of trust and harmony on the relationship between leadership style and its effectiveness, and to interpret the moderating effects of generation on the relationships between both paternalistic and transformational leadership with trust and harmony.

Most theories of leadership in organizational behavior originated in the United States and Western Europe and are hypothesized to be universally applicable to non-Western contexts. 
Departing from this tradition, the current study proposes a Chinese culture-specific leadership theory, built on traditional Confucianism. The principle aim is to examine and articulate a culturally informed and warranted ground for a leadership model in the Chinese context. The results of the study provide a new perspective on leadership in the Chinese context by focusing on three dimensions of paternalistic leadership (authoritative, benevolent, and moral leadership) that are ignored in the Western leadership literature. The results also suggest that trust in the leader and harmonious relationships in an organization are key mechanisms for explaining effective leadership in Chinese organizations regardless of whether paternalistic or transformational leadership is used. What's more, as the younger generation is becoming the dominant workforce, a successful leader in China should use Western practices and integrate them to fit in Chinese organizations in a way that also acknowledges Chinese traditions. 
Copyright 2012

by

Wai Kwan Lau 


\section{ACKNOWLEDGEMENTS}

This dissertation would not have been made possible without the great help of many individuals. First, I would like to thank my dissertation chair, Dr. Vicki Goodwin, for her exemplary mentoring, patience, and immense knowledge. Though she had so many other responsibilities, she always found time for me and taught me how to be a critical thinker and a skilled writer. The process of completing the dissertation embraces the challenges and frustrations. I would not have gone through this process without her guidance. I am also thankful to all my committee members, Dr. Lew Taylor, Dr. Manjula Salimath, and Dr. Robert Pavaur, for their endless support. They always provided me a lot of encouragement, guidance, and detailed feedback. I would also like to thank the faculties and staffs at the Department of Management for their help and support they have shown me during the Ph.D. program.

I am most grateful to my dear parents and my brother for their unconditional understanding, love, and help of data collection in China. My gratitude to them cannot be expressed in words. I am also fortunate to have very supportive family members, my husband, Wing-Sun $\mathrm{Ku}$, and my son, Aaron $\mathrm{Ku}$. I would not be able to pass through my ups and downs during the Ph.D. program without their love and support. I know they are very proud of my achievement today. 


\section{TABLE OF CONTENTS}

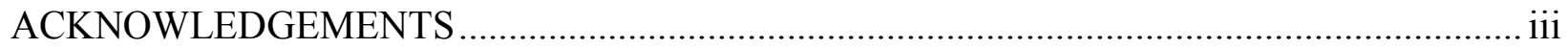

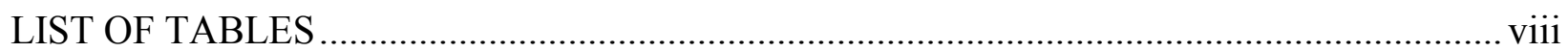

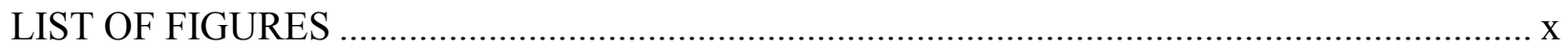

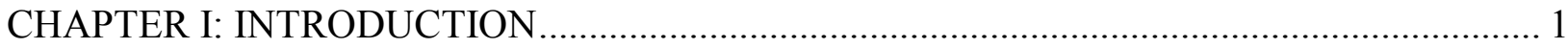

Research Background .................................................................................................... 1

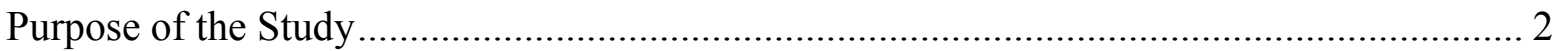

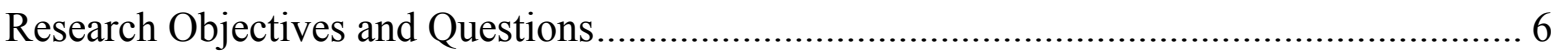

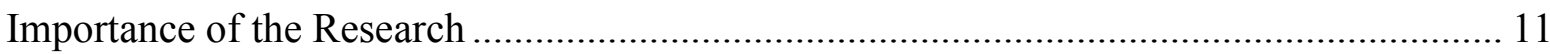

Benefits for Academic Research ........................................................................ 12

Benefits for Business and Industry ..................................................................... 13

Summary

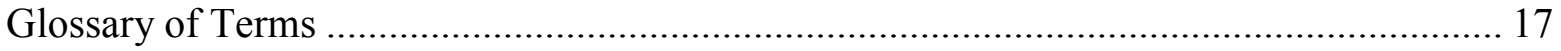

CHAPTER II: LITERATURE REVIEW AND HYPOTHESIS .................................................. 20

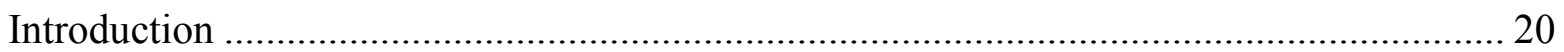

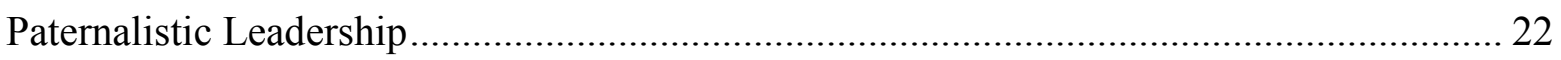

The Origin and Cultural Roots of Paternalistic Leadership ......................................... 22

The Existing Model of Paternalistic Leadership ......................................................... 26

Elaborations of Paternalistic Leadership .................................................................... 30

Transformational Leadership .......................................................................................... 33

Theoretical Framework of Transformational Leadership ........................................... 33

Outcomes of Transformational Leadership ................................................................ 35

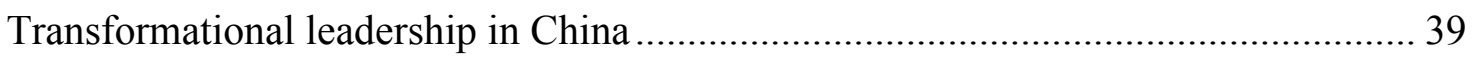

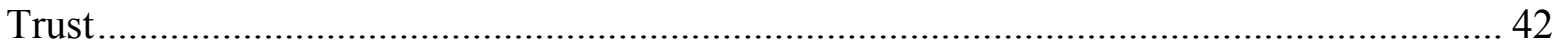




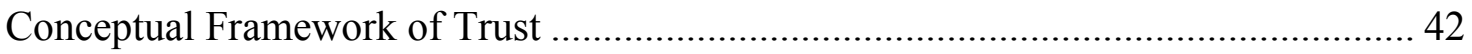

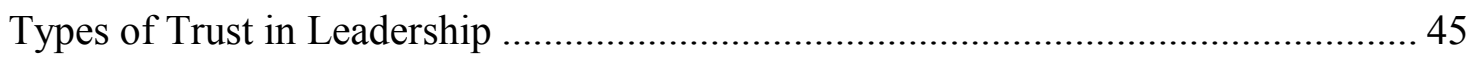

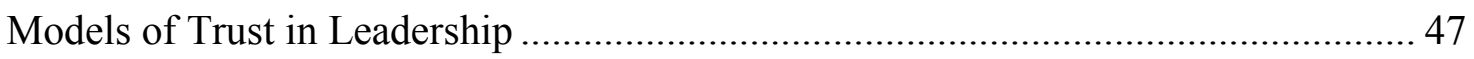

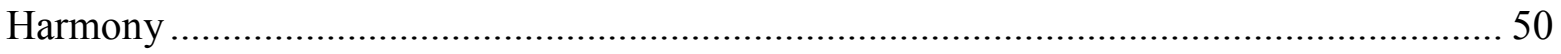

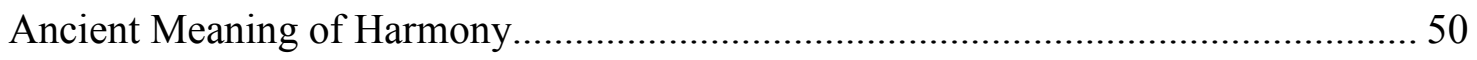

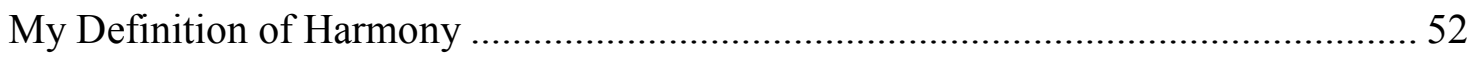

Relationship between Harmony and Chinese Leadership ............................................. 55

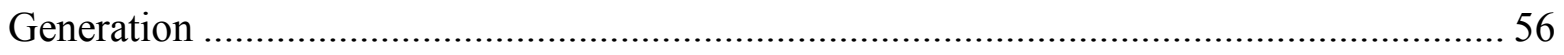

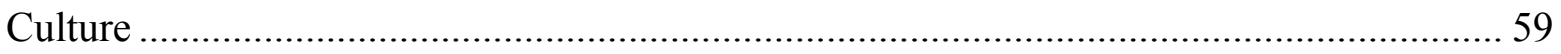

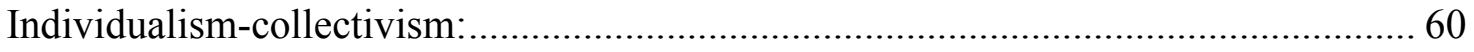

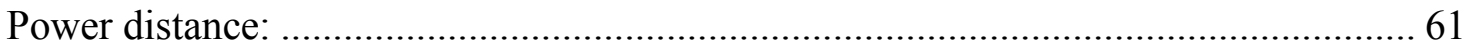

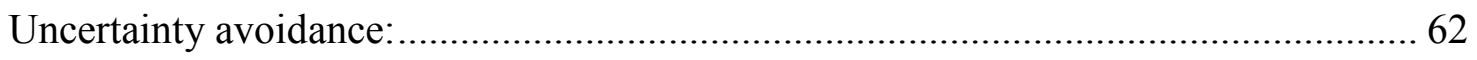

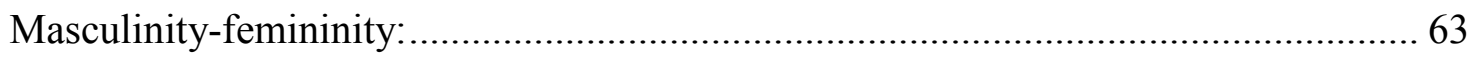

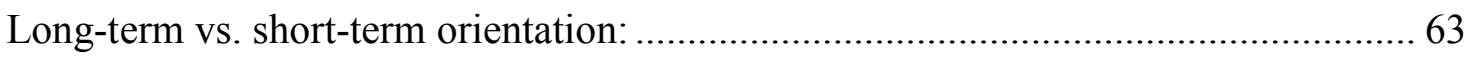

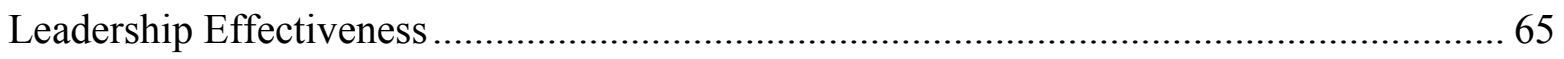

Overview of Theoretical Foundations and Hypotheses Development ................................... 67

Mediating Effect of Trust on the Relationship between Leadership Style and

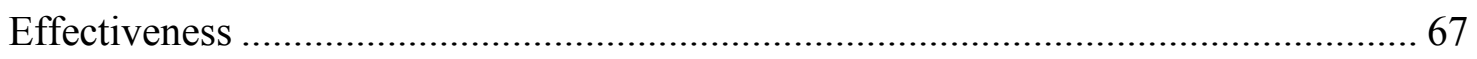

Mediating Effect of Harmony on the Relationship between Leadership Style and

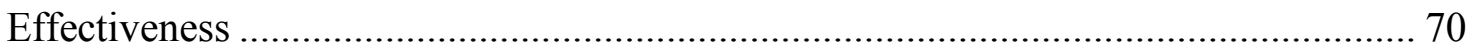

The Moderating Effect of Generation on the Relationship between Leadership Style

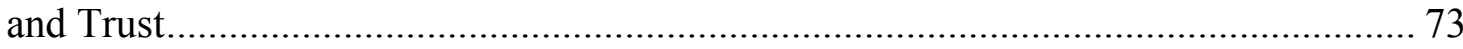

The Moderating Effect of Generation on Relationship between Leadership Style and

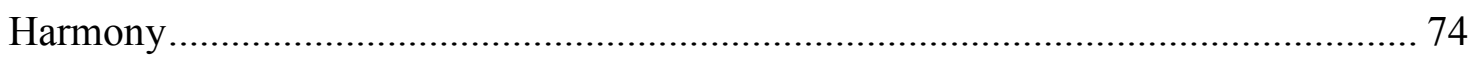

Summary

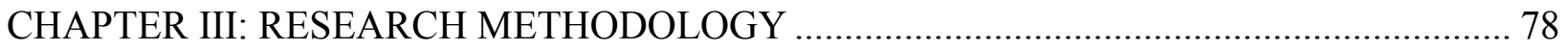




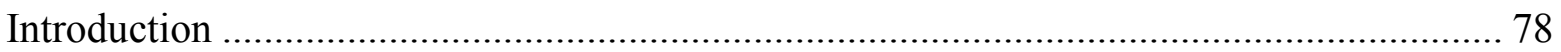

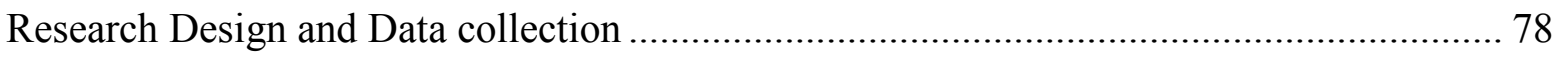

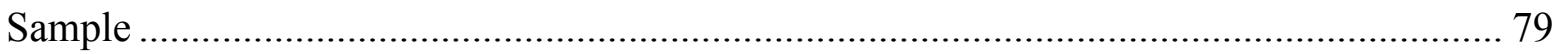

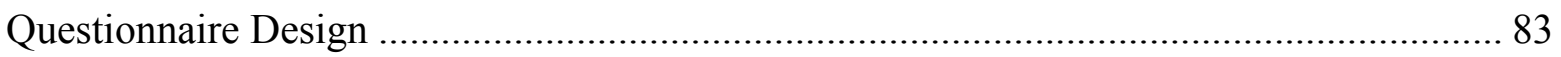

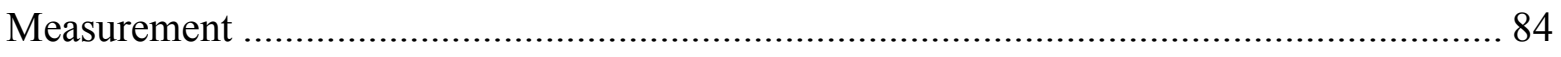

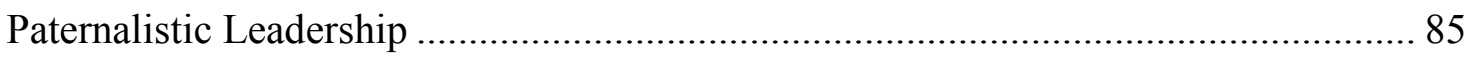

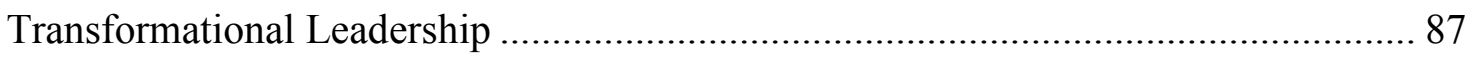

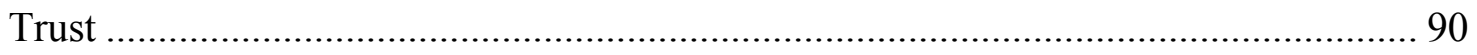

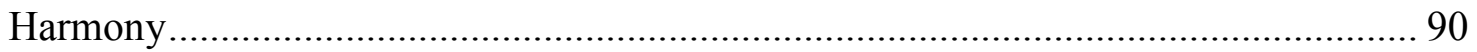

Leadership Effectiveness...................................................................................... 91

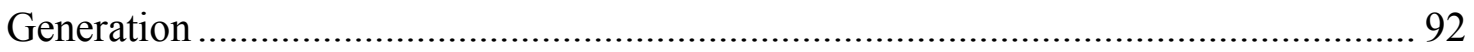

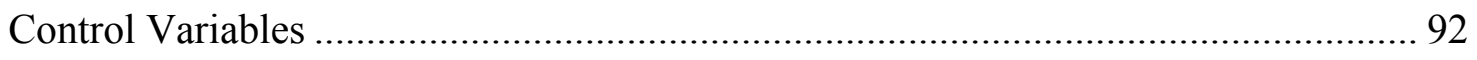

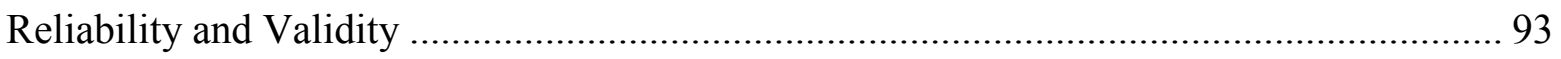

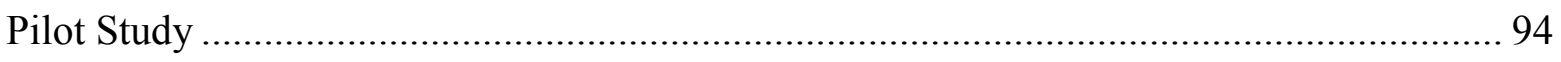

Demographic Characteristics of the Pilot Study Sample ............................................... 95

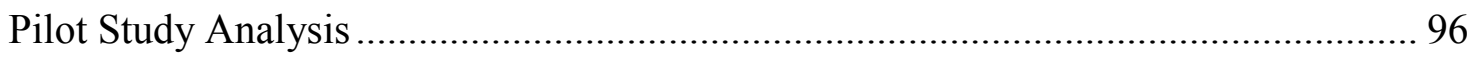

Summary and Revisions to the Main Study ................................................................. 112

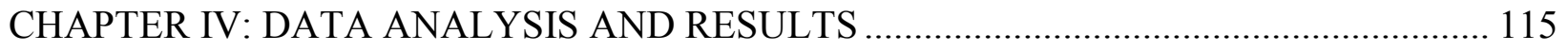

Survey Response Rate and Sample Characteristics.......................................................... 115

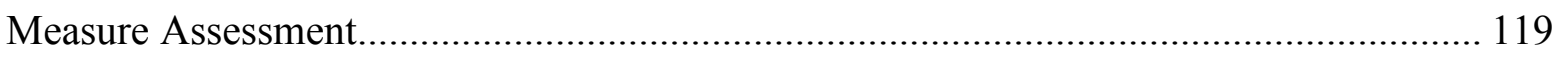

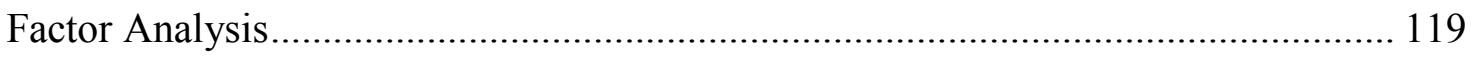

Reliability and Valididty Tests.......................................................................... 122

Intercorrelations between Variables..................................................................... 123

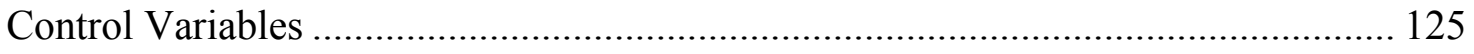

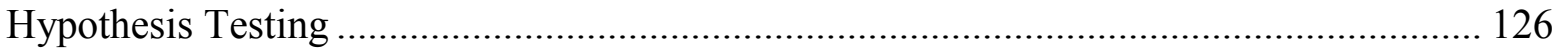


Summary

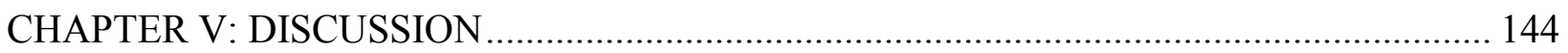

Implications of the Findings .......................................................................................... 144

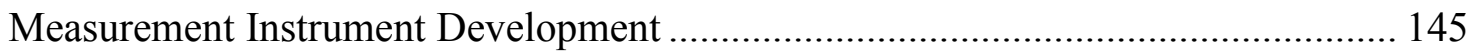

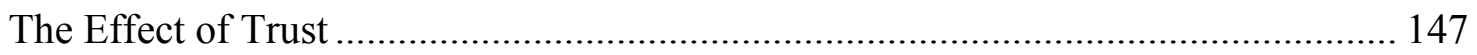

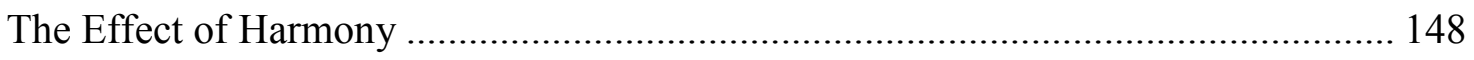

The Role of Generation ....................................................................................... 150

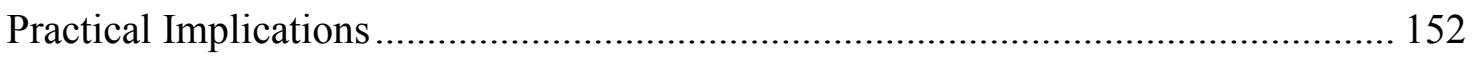

Contributions and Limitations of the Study ................................................................. 155

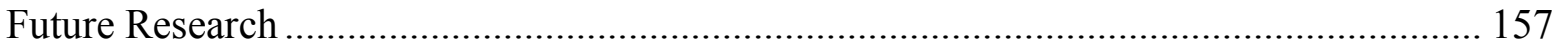

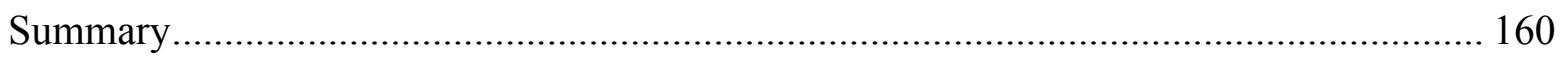

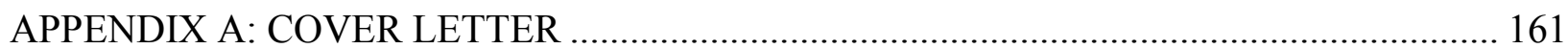

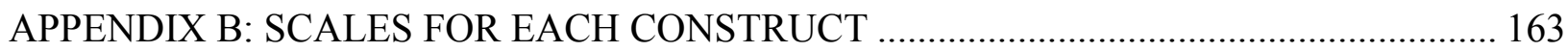

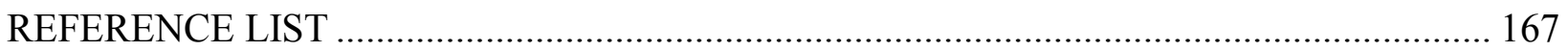




\section{LIST OF TABLES}

Table 1-a. Measures and Questionnaire Items for Paternalistic Leadership.................................. 88

Table 1-b. Measures and Questionnaire Items for Authoritarian Leadership................................ 89

Table 2. Measures and Questionnaire Items for Transformational Leadership ............................. 89

Table 3. Measures and Questionnaire Items for Trust.............................................................. 90

Table 4. Measures and Questionnaire Items for Harmony …………………………............... 91

Table 5. Measures and Questionnaire Items for Leadership Effectiveness .................................. 92

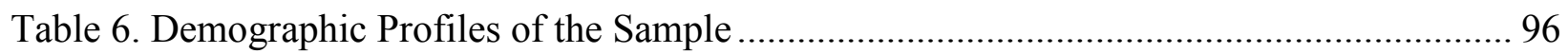

Table 7-a. Rotated Component Matrix of Paternalistic Leadership. ............................................. 98

Table 7-b. Rotated Component Matrix of Authoritarian Leadership ........................................... 98

Table 8. Rotated Component Matrix of Transformational Leadership........................................... 99

Table 9. Rotated Component Matrix of Harmony......................................................................... 100

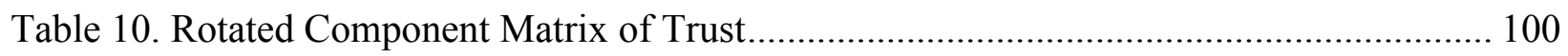

Table 11. Rotated Component Matrix of Effectiveness.......................................................... 100

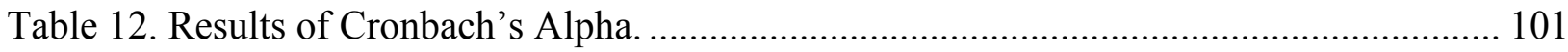

Table 13. Correlations Matrix of Paternalistic Leadership .......................................................... 102

Table 14. Correlations Matrix of Predictors and Outcomes …………………………............... 102

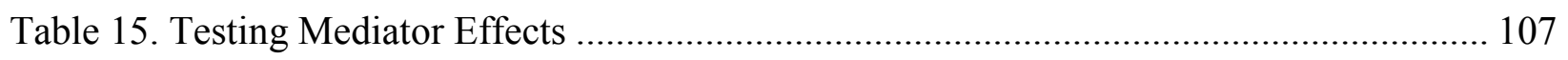

Table 16. Testing Moderator Effects ....................................................................................... 109

Table 17. Characteristics of the Basic Work of the Company..................................................... 116

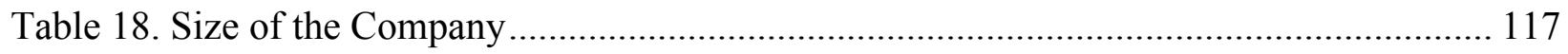

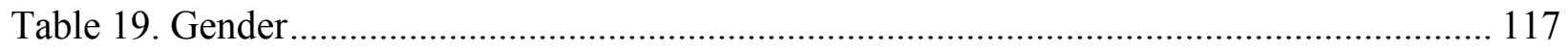

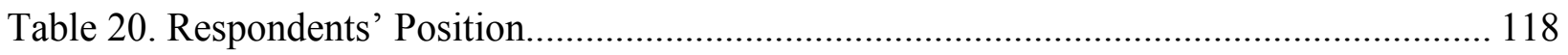

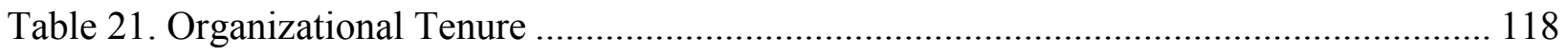


Table 22. Years Working with the Current Supervisor ....................................................... 118

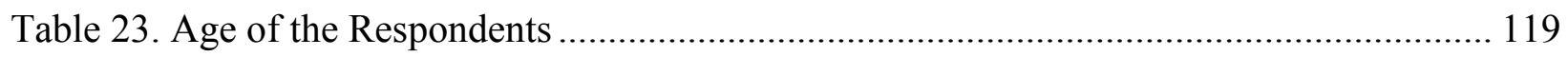

Table 24. Education of the Respondents................................................................................ 119

Table 25. Factor Analysis of Paternalistic Leadership ............................................................ 120

Table 26. Factor Analysis of Transformational Leadership ...................................................... 121

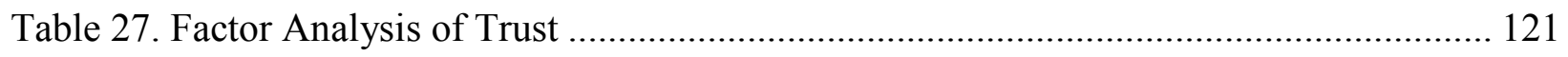

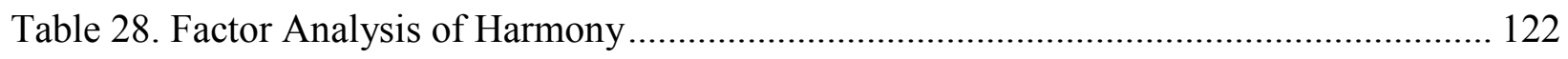

Table 29. Factor Analysis of Leadership Effectiveness........................................................... 122

Table 30. Correlations Matrix of All the Variables ............................................................... 124

Table 31. Correlations Matrix of Paternalistic and Transformational Leadership Items ........... 124

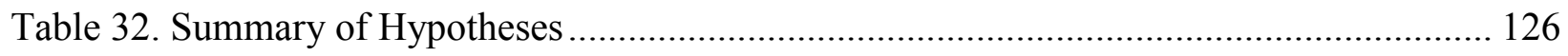

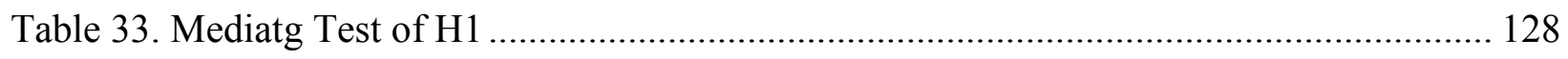

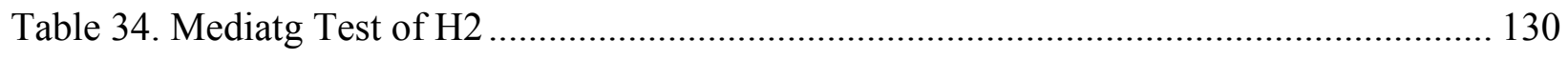

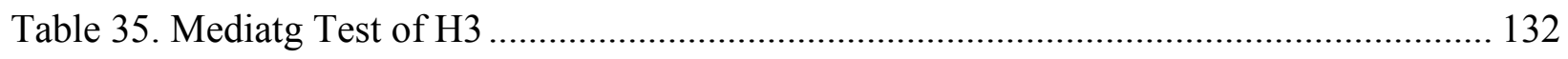

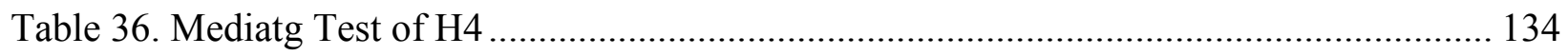

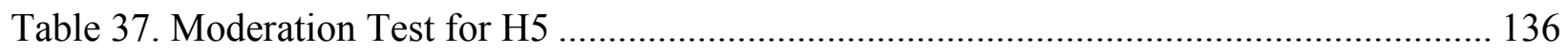

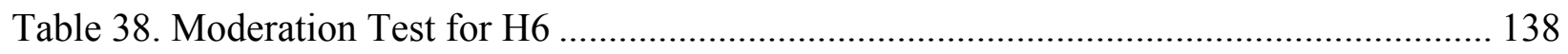

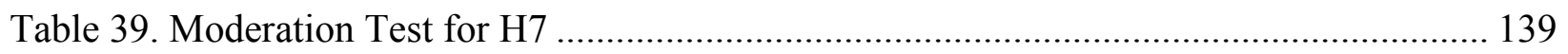

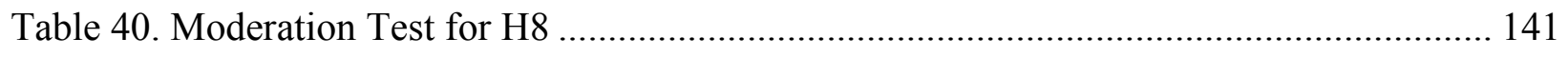

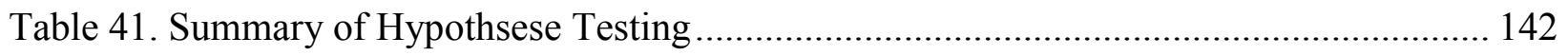




\section{LIST OF FIGURES}

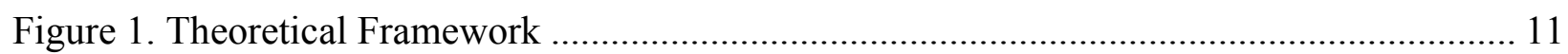

Figure 2. Flow Path of Research Process in this Study ............................................................ 16

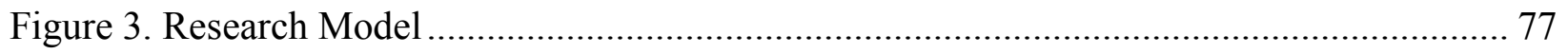

Figure 4. Diagram of Paths in Mediation Models.................................................................... 103

Figure 5. Moderator Effect of Generation on the Paternalistic Leadership-Trust Relationship. 112

Figure 6. Moderator Effect of Generation on the Paternalistic Leadership-Harmony Relationship

Figure 7. Moderator Effect of Generation on the Transformational Leadership-Trust Relationship 112

Figure 8. Moderator Effect of Generation on the Transformational Leadership-Harmony

Relationship. 112

Figure 9. Moderator Effect of Generation on the Paternalistic Leadership-Trust Relationship. 137

Figure 10. Moderator Effect of Generation on the Paternalistic Leadership-Harmony

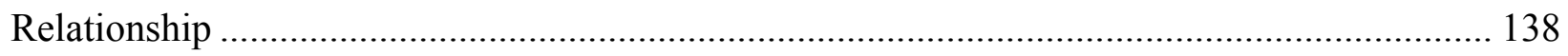

Figure 11. Moderator Effect of Generation on the Transformational Leadership-Trust

Relationship 140

Figure 12. Moderator Effect of Generation on the Transformational Leadership-Harmony

Relationship. 


\section{CHAPTER I}

\section{INTRODUCTION}

\section{Research Background}

Leadership has attracted a significant amount of scholarly attention in the past few decades. However, most research and theory contributions are to a great extent limited to accounting for leadership practice in the West (Littrell, 2002). As international trade and globalization have accelerated the process of interdependence for most nations in the world, effective leadership in different national contexts becomes a relevant and critical issue. From this perspective, it is important to study leadership in non-Western cultures and it is critically important to understand the extent to which the effectiveness of leadership varies depending on cultural differences between East and West.

Among non-Western countries, China is particularly interesting because 1) as one of the oldest civilizations in the world, China has broad philosophical and cultural underpinnings, including the teachings of Confucianism, Buddhism, and Daoism, which are found in the words of well-known Chinese writers like Sun Zi, Confucius, Mencius, Lao Tzu, and Chuang Tzu; 2) with the largest population in the world, China sparks a lot of leadership ideas as well as practices; however, among the vast volume of literature on leadership within and outside China, there is little research on Chinese leadership, and little conducted by Chinese scholars (Pellegrini \& Scandura, 2008); 3) at a time when China is becoming more central in a globalized world economy, business managers as well as scholars from outside China increasingly feel the importance of understanding the thoughts and views of Chinese leadership and management.

China has conducted political and economic reforms since the 1980s. The country is the largest receiver of foreign direct investment during the first years of the $21^{\text {st }}$ century (UNCTAD, 
2002). Many companies are developing subsidiaries and joint ventures in China to lower costs and participate in the global marketplace. Long term success in China depends on how well the managers understand and interact with the workforce (Gallo, 2008). Therefore, studying Chinese leadership helps to gain insight into its role in China's economic takeoff.

Moreover, with a massive influx of foreign capital and investment, Western management ideas and practices also have flowed into the Chinese territory, influencing Chinese leadership behaviors (Wah, 2009; Chen \& Farh, 1999). These Western leadership ideas could compliment or clash with traditional Chinese leadership philosophy (Gallo, 2008). Examining leadership from both cultures could spark new leadership ideas and give rise to new leadership practices. There are cultural differences between China and the West in the way workers view their leaders, what they expect from their leaders, and what leaders can expect from their workforce. Furthermore, there are very fundamental differences in how life works in China compared to the West. Thus, investigating Chinese leadership not only helps to advance our knowledge of leadership in the Chinese context but also informs the study of global leadership with implications for global organizations.

\section{Purpose of the Study}

This study is designed to develop an effective leadership model that works in the Chinese context. Paternalistic leadership, a dominant leadership style in an Eastern business environment, is compared with transformational leadership, a dominant leadership style in a Western business environment. The notion of transformational leadership was developed under the tutelage of Bernard Bass (1998). Transformational leaders define the need for change, develop a vision for the future, and mobilize follower commitment to achieve results beyond what would normally be expected (Avolio \& Bass, 1988). Transformational leadership is found 
to be compatible with collectivistic values (Walumbwa \& Lawler, 2003) and is believed to be appealing and generalizable to Chinese leadership situations (Chen \& Farh, 1999). Other researchers have found that within Chinese organizations, leader behaviors are quite distinct from transformational leadership, referring to this leader style as paternalistic leadership (Redding, 1990; Cheng, 1995). Much of paternalism is rooted primarily in Confucianism in terms of the emphasis on respect for hierarchy, benevolence, and the role of morality (Silin, 1976; Westwood, 1997; Farh \& Cheng, 2000). The questions are asked, "Transformational or paternalistic leadership, which one is more effective in Chinese organizations? Is one type of leadership superior to the other one in the Chinese culture?"

Researchers of Chinese leadership have undertaken many studies both on transformational and paternalistic leadership (Chen \& Farh, 1999; Javidan \& Carl, 2005). Studies reveal that no matter what type of leadership, for it to be effective in Chinese organizations, the leader style should match Chinese cultural features (Westwood, 1997; Pellegrini \& Scandura, 2008). The cultural differences between China and the West relating to leader-follower relationships suggest different leadership behaviors may be more effective in one of these cultures than in the other.

For example, trust in the West is treated as the expectation that arises within a community of regular, honest, and co-operative behavior (Lewicki \& Wiethoff, 1995). Trust is required in a company for the business leader and the others to work well together. In China, however, trust is much more of a personal one. Personal trust needs to be earned rather than be more automatic as it is in the West. Chinese employees often have to guess their leaders' meaning of what they are saying. Unless a strong level of trust is obtained, there may be a guessing game played at work to determine the true meaning of the leader's statements (Littrell, 2002). 
These different approaches of trust may be due to culture. Different from low context culture countries where people tend to be more precise, specific and require more specific information to communicate (Hall, 1976), China usually is categorized as a high context culture country where people place great emphasis on friends and networks and are less formalistic in negotiations (Hall, 1976). People in high context cultures rely on analysis and common background rather than words to explain situations, what people say does not represent what they really mean (Hall, 1976). A Chinese leader who asks for an employee's opinion may not really be looking for an employee's ideas; because he or she expects the employee's support of his or her opinions. This implicit communication causes different trust situations in China when compared with the West. Therefore, it is important to examine how the Western versus the Chinese view of trust differs and how trust in each culture relates to leadership effectiveness?

Another factor highly prized by Chinese traditions and associated with Chinese leadership is harmony (Bond \& Hwang, 1986). The best Chinese leaders display a distinctive and effective way of negotiating complex environments. They do so in such a way as to create a harmonious result in which all parties are at least reasonably satisfied with the result (Gallo, 2008). Achieving the degree of harmony involves complex influence strategies designed to bring each party to support the same solution. Chinese leaders construct and present several alternatives in such a way that each party will voluntarily choose the same alternative, the one the leader sees as best overall (Wah, 2009). Care is taken to ensure that no party fully wins at the expense of others. The foundation of this harmonious relationship in an organization is an assumption that the relationships will be long-lasting, that is, it is in no one's interest for one side to be seen as totally winning (Westwood, 1997). This study will, therefore, also explore how harmony mediates the relationship between leaders' behavior and leadership effectiveness. 
Moreover, employees' individual differences such as generation should be considered as an important factor that will impact the outcome as people in different generations have different values and beliefs, thus viewing leaders differently. Compared with Generation X (those individuals born from 1965 to 1979), Generation Y (individuals born from 1980 to 1994) is less cynical, more optimistic, and more idealistic. While Generation X, with collectivistic characteristics, has an unquestioning acceptance of hierarchy and trust authority, Generation Y, with individualistic values, is full of confidence in speaking and interacting with their leaders. China is characterized as a collectivistic culture, with high power distance, high uncertainty avoidance, as feministic, and with a long-term orientation (Hofstede, 1991). These cultural characteristics as well as Confucianism influence Chinese organizational behaviors and shape leadership in certain ways. The Confucian notion of the father-son relationship and high power distance of hierarchy are deeply implanted in Chinese leadership practices when decision making is typically centralized, and followers expect their leaders to make decisions. Once these decisions are made, the followers follow their leaders' directions obediently and without question (Chen \& Farh, 1999). Leaders are expected to be personally engaged with their subordinates and subordinates are expected to sacrifice their weekends and even vacations if their organizations want them to (Westwood, 1997). While exploring each of the cultural dimensions as well as Confucianism, one should keep in mind that these cultural elements may work side by side in shaping leadership. Examining them in context will help us to understand how trust and harmony can be influenced by cultural factors in leadership practices, and consequently impact leadership effectiveness.

In summary, the purposes of this study are to clarify the mediating effects of trust and harmony on the relationship between leadership style and its effectiveness, and to interpret the 
moderating effects of generation on the effectiveness of both paternalistic and transformational leadership. Most theories of leadership in organizational behavior originated in the United States and Western Europe and are hypothesized to be universally applicable to non-Western contexts. Departing from this tradition, I attempt to propose a Chinese culture-specific leadership theory, built on traditional Confucianism. The principle aim is to examine and articulate a culturally informed and warranted ground for a leadership model in the Chinese context. The model in this study provides a new perspective on leadership in the Chinese context by focusing on three dimensions of leadership (authoritative, benevolent, and moral leadership) that were ignored in the Western leadership literature. I also attempt to investigate how best Western leadership practice combines with traditional Chinese wisdom to do a better job in accounting for the leader-follower dynamics in contemporary Chinese society.

\section{Research Objectives and Questions}

The purpose of conducting this research is stated in the previous section; here I specify the objectives of this dissertation as follows:

1. Review the research literature on paternalistic leadership, transformational leadership, culture, trust, harmony, generation, and leadership effectiveness. Document the major findings in these areas.

2. Relate relevant theories to enable the development of a hypothesized model for later empirical testing.

3. From the results of empirical testing, compare and verify the hypothesized relations.

4. Provide a discussion of results relative to the hypothesized research model in terms of the results obtained of each hypothesized relationship, differences in the findings from my expectations, theoretical connections, applications and implications for future research. 
There are seven major theoretical foundations in this dissertation: paternalistic leadership, transformational leadership, trust, harmony, generation, culture, and leadership effectiveness. The central research theme is the relationship between leadership style and its effectiveness in the Chinese context. Through the literature review, I will summarize the constructs and their relationships to one another. The first research question to be addressed is the following:

Research Question 1: What relevant theories and empirical findings of each construct (paternalistic leadership, transformational leadership, trust, harmony, generation, culture, and leadership effectiveness) are examined in the leadership literature?

Regarding the leadership style, some argue that transformational leaders stimulate followers to realize the important meaning of the tasks they are responsible for, motivate their high level needs for growth and development, establish a climate of mutual trust, stir their employees to look beyond their own self-interests for the good of the group, and achieve performance beyond expectations (Bass, 1985). Therefore, transformational leadership has a positive effect on subordinates' job performance in China (Chen \& Farh, 1999; Wang, Law, Hackett, Wang \& Cheng, 2005). Other researchers suggest that China has its own characteristics because of the existence of its unique cultural factors. They demonstrated that there is one unique style of Chinese leadership, paternalistic leadership, which matches better with Chinese traditional values than other types of leadership (Westwood, 1997; Cheng, 1995). This issue of leadership style leads to the second research question:

Research Question 2: Which fits better in the Chinese context - Paternalistic or Transformational leadership?

Although Chinese leadership receives increasing attention in management literature, there is still considerable disparity among authors with respect to the definition and effectiveness of Chinese leadership. The three-dimensional construct of paternalistic leadership developed by Cheng, Chou, and Farh (2000) perhaps is the most widely used leadership construct in China. 
Nevertheless, a review of studies related to paternalistic leadership ( Pellegrini \& Scandura, 2008; Aycan, 2006; Cheng, Shieh, \& Chou, 2002b) reveals the unfortunate fact that measurement tools developed for paternalistic leadership suffer from inadequate reliability and validity. One of the dimensions, authoritarianism, is found to negatively correlate with the other two dimensions, morality, and benevolence (Cheng et al., 2000). In turn, authoritarianism is found to negatively relate to subordinate attitudinal and behavioral outcomes, whereas both benevolent and moral leadership are positively associated with the outcomes. Consequently, researchers have to study the three constituent dimensions separately rather than treat paternalistic leadership as a unified construct (Pellegrini \& Scandura, 2008; Aycan, 2006). In addition, harmony is a concept mentioned frequently by researchers that is related to Chinese leadership. The best Chinese leaders are observed to display a distinctive and effective way of seeking harmony in organizations (Gallo, 2008); however, there is no clear definition of harmony in the literature and, thus, no valid and reliable measurement instrument. Therefore, these issues lead to the third research question:

Research Question 3: How can the constructs of paternalistic leadership and harmony be refined and measured to promote for future research?

Embedded in a social influence process, leadership exists all around the world. Therefore, the notion and the construct may vary across different cultures (Hofstede, 2001). According to Hofstede (2001), China is a country with high power distance, high collectivism, high concern for long-term results, and under deep influence from Confucianism, which advocates social harmony and particularly, harmonious interpersonal relationships. This view also extends to the managerial process. Chinese leaders believe that relationships should be hierarchically maintained and harmony within the organization should be achieved even at the expense of less productive performance. Cultural factors are relevant for this study because leadership is about 
hierarchical relationships between the leaders and the follower. The cultural differences in hierarchical relationships require their consideration in leadership research. Thus, this leads to the fourth research question:

Research Question 4: How does Chinese culture affect the effectiveness of leadership? According to Podsakoff et al. (1990), subordinates' trust in the leader is one of the most important variables that can mediate the effectiveness of transformational leadership in terms of producing performance outcomes. Being trusted by one's followers may create an obligation or responsibility on the part of the leader to enable or empower a follower to perform. Chen and his colleges (Chen, Eberly, Chiang, Farh, \& Cheng, 2011) found that trust signifies a strong personal bond and positive emotions and this solicited trust from the subordinates can help paternalistic leaders achieve a positive impact in the Chinese context. Trust plays a critical role in explaining how paternalistic leaders can motivate followers to meet high performance standards (Chen et al., 2011).

Other researchers (Westwood, 1997; Redding, 1990) found that harmony, a concept closely related to Confucianism, also is critical for shaping leadership. Leadership in Confucian-based societies emphasizes paternalism and benevolence, preserves interpersonal harmony, and that harmony is highly valued. Harmonious relationships within an organization help employees to be more collectivistic and focus on group-level goals; hence, it fosters the effectiveness of transformational leadership (Zhang et al., 2008). In contrast to organizations with no harmonious relationships between the leader and his/her followers, organizations with harmonious relationships are likely to minimize the negative work attitudes and increase cohesion of the work team, which in turn helps to keep the organizations running smoothly and making profits (Zhang, Chen, Liu \& Liu, 2008). 
As China is becoming westernized, young people are modernizing also. The large population of the young generation makes it essential to understand Generation Y because they make up almost fifty percent of China's workforce. As they move from school into jobs, organizations have noticed that this generation makes different demands and needs to be motivated in new ways (Elegant, 2007). Different from people of Generation X who comply with hierarchic rules and authority, people from Generation Y are more independent, cynical, and demanding. Meanwhile, competitive pressure also forces Chinese businesses everywhere to demand more contributions from their employees. Nowadays, employees are frequently required to be responsible for the business results achieved, rather than to follow a narrow set of guidelines or seek permission for variance (Hempel \& Chang, 2002). People from different generations may think differently about business and how people should lead them. Some of the old traditional leader behaviors are so outdated that they tend to be rejected by most subordinates today. It is interesting to employ an integrative dynamic rather than a kind of static perspective to explore the relationship between leadership style and effectiveness of leaders. These thoughts center around the research question below:

Research Question 5: How do trust, harmony, culture, and generation impact the relationship between leadership style and it effectiveness?

Figure 1 presents the preliminary theoretical framework of this dissertation. Specifically, I propose to examine the relationship between leadership style and effectiveness. My literature review will incorporate a discussion of trust and harmony as mediators of the leadershipeffectiveness relationship. Further, I will address generation and culture as potential moderators of this relationship. This study addresses and discusses the five culture dimensions (individualism vs. collectivism, power distance, uncertainty avoidance, masculinity vs. femininity, and long-term vs. short term orientation) developed by Hofstede $(1991,2001)$. It 
aims to provide a cultural understanding of how the roots of Chinese wisdom relate to current business leadership. My research model does not include culture as a moderating factor. As an initial step in the test of the theory, I will collect data only in Chinese companies. This effect will allow me to examine my hypotheses within a single culture before broadening data collection to other cultures allowing for a full test of the model.

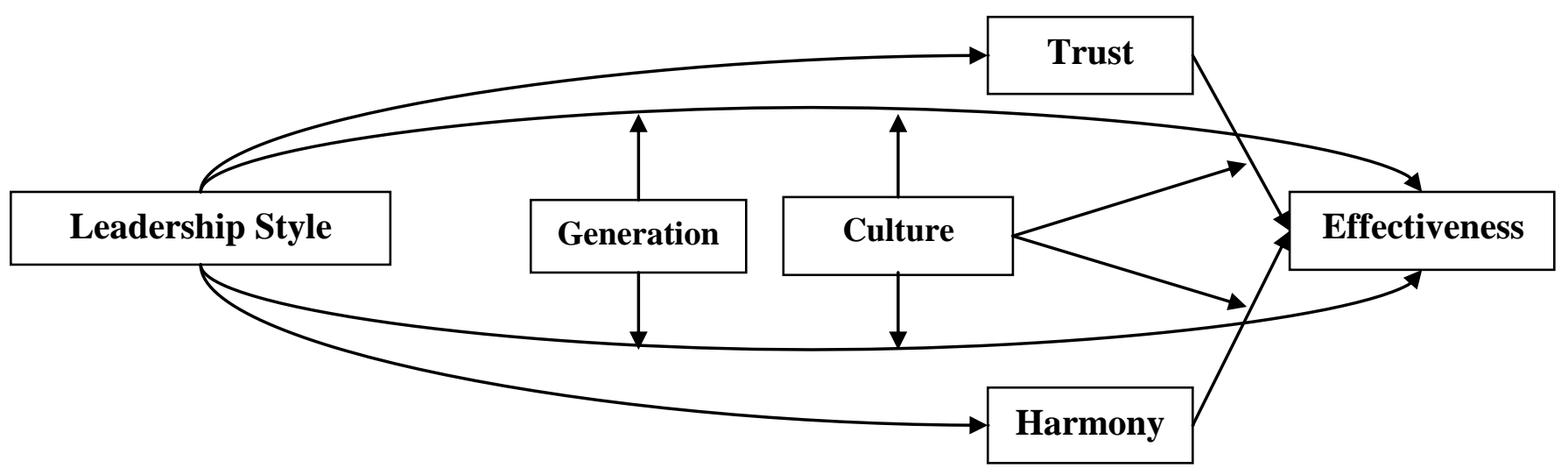

Figure 1. Theoretical Framework

Importance of the Research

This study of leadership style, trust, harmony, generation, culture and effectiveness addresses a need in both the academic and business communities. General contributions to academic research include 1) the enhanced understanding and completion of the leadership process as a dynamic process; 2) initial empirical test of the context of cultural effects on the leadership process; 3) an insightful examination of trust and harmony; and 4) an insightful examination on how changes in generation will impact the perception of leadership effectiveness. Likewise, general contributions to business and industry include a clearer understanding of how to blend best Western practices with Chinese traditional values to achieve the most effective leadership in the Chinese context. The specific benefits of this study follow. 
Benefits for Academic Research

This study will contribute to the literature on leadership by investigating how trust mediates the relationship between leadership style and performance for both paternalistic and transformational leadership. In China, trust is extremely strong in the family and among close friends. The Chinese have a more difficult time than Westerners becoming corporate professional managers because of their inclination to deeply trust only people with whom they have a very close relationship (Fukuyama, 1995). The implication for leadership is that Chinese employees will be slow to trust their leaders. Thus, Chinese employees will hesitate to follow their leaders' instructions if there is not enough trust held (Gallo, 2008). Regardless, trust in leadership is greatly understudied in the Chinese culture when compared with the Western culture. This study highlights the importance of understanding trust in different cultures and its mediating effects in actual organizational settings.

Furthermore, this study also addresses the need to include cultural factors, such as traditional Chinese values, when examining the leadership process. In the traditional Chinese culture, Confucianism has shaped the Chinese mind and influenced leadership behaviors. In this study, I will develop a new research construct, harmony, based on the Confucian notion. It is proposed that harmony mediates the relationship between leadership behaviors and effectiveness. Not only paternalistic leaders, but also transformational leaders need to consider the cultural effect of harmony in Chinese organizations.

An additional contribution relates to the measurement of paternalistic leadership using authoritative leadership to replace the previous authoritarian leadership dimension of paternalistic leadership. Authoritative leadership refers to leader behaviors that rely mostly on legitimate authority and professional expertise to influence subordinates (Aycan, 2006). Authoritarian leadership is found to be negatively correlated with outcome variables as well as 
the other two dimensions (benevolence and morality) of paternalistic leadership (Aycan, 2006). Using authoritative leadership; on one hand, can diminish the negative associations of authoritarian leadership, on the other hand, it still characterizes a leader with strong legitimate authority, control, and obedience from subordinates. The intent is to resolve the emergent conceptual problems related to paternalistic leadership.

In addition, the leadership process is investigated from a dynamic view by examining the moderating role of generation. This study explores Chinese leadership research by examining the cultural differences across generations and how each generation views leaders differently. It would be interesting to know how the impact of generational differences may reveal what generation would be better fit what type of leadership style.

Benefits for Business and Industry

The study will provide empirical evidence to help advance and refine the framework for studying the relationships among leadership style, trust, harmony, generation, culture, and effectiveness. This study of Chinese leadership reinforces the call for relationship building by global leaders (Pellegrini \& Scandura, 2008). In the context of the Chinese culture, relationship building should benefit from trust and harmony when they are consistent with Confucian ethics. Yet, even though Confucian ethics can translate into relationship-building skills, Western managerial concepts should be emphasized in leadership practices in the Chinese context also. With rapid societal modernization, there has been an accompanying change in social values. Western values that flow into China and are accepted by Chinese employees, especially those born after 1980 (Generation Y), along with a massive influx of foreign investment, should be examined and considered by Chinese leaders. As China integrates itself more and more into the global economy, the younger people in China lean more towards Western approaches than do old 
people, and the companies begin to behave more like those that are dominant in the West. Dynamic interchange between East and West suggests that successful Chinese leaders should continue to modify Western practices to make them fit better in China than they otherwise would. This research will provide a depth information about how Chinese leaders should cooperate with the two-way interchange to be effective.

\section{Summary}

This chapter introduced the research, and provided an overview of the study. It also describes the purposes and objectives of the study, the structure of the dissertation, and the implications that this research has for both academic study and business. This work will provide more holistic and insightful information useful for understanding Chinese leadership. The research procedures of this study are shown in Figure 2.

This dissertation contains five chapters and the summary of each is shown as below:

In Chapter I, I outline the research background, objectives, conceptual framework, importance of the research, and path flow of this dissertation. A theoretical model is proposed.

In Chapter II, I review the related literature relevant to this study. This chapter includes seven major research streams: paternalistic leadership, transformational leadership, trust, harmony, generation, culture, and leadership effectiveness. Key constructs and their relationships are identified. In addition, research hypotheses are developed. Finally, a hypothesized research model of this study is proposed at the end of this chapter.

In Chapter III, I describe the research design and methodology employed for this dissertation. Construct measures, survey questionnaire items, data collection procedures, and the sampling method are addressed in this chapter. In the end, I conduct a pilot study for validating the newly developed questionnaire instrument. 
In Chapter IV, I present a detailed description of the analysis and results based on statistical techniques proposed in Chapter III. This chapter describes the procedures of preparing the data for analysis, the performance of the measures utilized, the testing of the hypotheses, and the post hoc analyses employed.

In Chapter V, I address the summary of significant findings and conclusions of this dissertation. Managerial implications, contributions to existing research, and suggestions for future research will also be presented in this chapter. 
Identify Research Objectives and Theoretical Model (Ch. I)

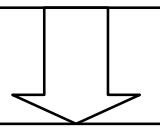

Literature Review (Ch. II)

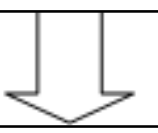

Develop the Theoretical Foundations and Constructs (Ch. II)

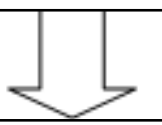

Develop the Hypothesized Research Model (Ch. II)

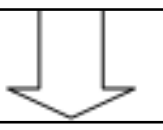

Research Design and Instrument Development (Ch. III)

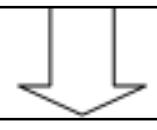

Survey Distribution and Data Collection (Ch. III)

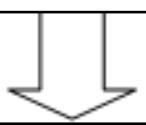

Data Analysis (Ch. IV)

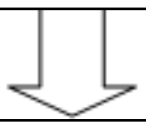

Testing Hypotheses and Proposed Relationships (Ch. IV)

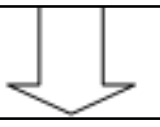

Conclusion and Suggestions (Ch. V)

Figure 2. Flow Path of Research Process in this Study 


\section{Glossary of Terms}

The following is a list of terms and their conceptual definitions:

Paternalistic Leadership: paternalistic leadership is a type of leadership that combines strong and clear authority with concern, considerateness, and elements of moral leadership (Silin, 1976). Paternalistic leadership consists of three dimensions: authoritarianism, benevolence, and moral leadership (Farh \& Cheng, 2000).

Authoritarian leadership refers to a leader's behavior of asserting strong authority and control over subordinates and demanding unquestioned obedience from subordinates. Benevolent leadership implies that a leader demonstrates individualized, holistic concern for subordinates' personal and familial well-being.

Moral leadership is broadly depicted as a leader's behavior that demonstrates superior moral character and integrity in behaviors such as not acting selfishly (especially refraining from abusing authority for personal gain) and leading by example.

This study adopts the construct of authoritative leadership in place of authoritarian leadership because the term of authoritative leadership is not only more consistent with the indigenous perspectives of high power distance cultures in China (Hofstede, 2001) but also better matches the Chinese organization where strong legitimate authority and strong technical expertise and business acumen are highly processed (Redding, 1990). Authoritative leadership refers to a leader's behavior of relying primarily on legitimate authority and professional expertise to influence subordinates (Aycan, 2006).

Transformational Leadership: transformational leaders inspire followers to transcend their own self-interests for the good of the organization and are capable of having a profound and extraordinary effect on their follower (Judge \& Piccolo, 2004). This study uses Podsakoff, MacKenzie, Moorman and Fetter's (1990) conceptualization of transformational leadership 
because it is behaviorally oriented, well validated, and has been used in both North American and Chinese cultures (Fath \& Cheng, 1999). The six dimensions of transformational leadership are: 1) identifying and articulating a vision; 2) providing an appropriate model; 3) fostering the acceptance of group goals; 4) expressing high performance expectations; 5) providing individualized support; and 6) offering intellectual stimulation.

Trust: trust implies a belief that an individual will not act opportunistically or in a selfserving manner (Hall, Blass, Ferris, \& Massengale, 2004). It is a belief of a congruence of values and is conceptualized as a psychological state comprising the intention to accept vulnerability based upon positive expectations of the intentions or behavior of another (Rousseau, Sitkin, Burt, \& Camerer, 1998).

Harmony: based on the Confucian view, harmony in this study refers to a state of equilibrium where all things develop side-by-side without doing harm to one another, and finally lead to an optimal result. Harmony consists of three dimensions: order, relationship, and execution. Order refers to the institutional order, that is, juniors and seniors have their ranking. The relationships within an organization should be hierarchically maintained. Relationship refers to the concrete rules specifying the right relations with one another. Execution refers to a formal condition of search for right. It is the will-to-right. It refers to a leader's behavior of discovering and following the right way to make the concrete rules.

Generation: generation refers to a cluster of people born in the same year or in a certain period of time, who share similar values, attitudes, and life styles shaped by the particular epoch, thereby representing the characteristics of that specific era (Rogler, 2002). Generation in this study is categorized into two groups: Generation $\mathrm{X}$ and Generation $\mathrm{Y}$. Generation $\mathrm{X}$ refers to 
individuals born from 1980 to 1994 . Generation Y refers to individuals born from about 1965 to 1979 (Dembo, 2000; Ellen, Kerwin, \& Kerwin, 1990).

Culture: culture refers to collectivities in which the members share several psychological commonalities-assumptions, beliefs, values, interpretations of events (meanings), ideas, social identities, and motives-and abide by a set of shared norms in a common manner (Hofstede, 1991). The literature review focuses primarily on the four dimensions developed by Hofstede $(1980,2001)$ as these have been studied most extensively to date and are generally presumed to necessitate leadership approaches in different culture clusters (Jackofsky, Slocum, \& McQuaid, 1988; Hofstede, 2001). The four dimensions are: individualism-collectivism, power distance, uncertainty avoidance, masculinity-femininity, and long-term vs. short-term orientation.

Leadership Effectiveness: leader effectiveness implies the leader's contribution to the quality of group processes as it is perceived by followers (Yukl, 2006). Effective leaders motivate and enable their subordinates to reach shared goals (Bass \& Avolio, 1994). In this study, leadership effectiveness is examined in terms of the consequences of leaders' action for followers, and how it is perceived by followers. 


\section{CHAPTER II}

\section{LITERATURE REVIEW AND HYPOTHESIS}

\section{Introduction}

The study of leadership is one of the most often researched management topics (Ergeneli, Gohar, \& Temirbekova, 2007). The study of leadership theory and effectiveness has evolved from the trait phase to the behavioral phase, and to the contingency phase (Robbins \& Judge, 2007). In the 1980s, management researchers began an examination of leaders who transform organizations, referred to as transformational leaders (Robbins \& Judge, 2007). Transformational leaders are more effective not only because they themselves are more creative, but also because they encourage those who follow them to be creative, too (Jung, 2001; Shin \& Zhou, 2003). Followers of transformational leaders are more likely to pursue ambitious goals, be familiar with and agree on the strategic goals of the organization, and believe that the goals they are pursuing are personally important (Bono \& Judge, 2003). Nevertheless, researchers of leadership find that within Chinese organizations, leader behavior is quite different than that formed in Western cultures (Silin, 1976; Farh \& Cheng, 2000). Owing to the differences of culture, the eastern Chinese societies developed paternalistic leadership theory, which depends on traditions of the Chinese culture.

Trust represents a consistency of ideas, communication, and action between individuals, which inspires people to transcend or allay their fears (Tyler \& Degoey, 1996). Trust in and loyalty to the leader plays a critical role in western leadership, such as in the transformational leadership model (Podsakoff, Mackenzie, Moorman, \& Fetter, 1990). In the Chinese culture or a Chinese organization, trust in and loyalty to one's supervisor is also very important. It indicates identification with and support for the supervisor and the willingness to actively cooperate and 
assist with the supervisor's business (Littrell, 2002). Thus, trust is viewed as playing an important mediating role in the paternalistic leadership process.

In addition to trust, harmony also is important in the Chinese culture. Harmony is based on the Chinese tradition of Confucianism, and it is understood as a state of equilibrium where all things develop side-by-side without doing harm to one another, leading to an optimal result (Wah, 2009). An effective Chinese leader seeks harmony in business relations and displays a distinctive and effective way of negotiating complex environments. Achieving this degree of harmony involves complex influence strategies designed to bring each party to support the same solution (Westwood, 1997). A Chinese leader will think about all sides of an issue before making a decision, repeat this decision making process over and over, and ensure that no party fully "wins" at the expense of others (Gallo, 2008).

Starting in the 1980s, China developed its one-child policy as a means of population control. This policy marked a new generation of Chinese, Generation Y. Many of these youth developed an attitude that they were the center of the world. Compared to their parents, these young people have a higher education, are tech-savvy, more optimistic, independent thinking and express their own ideas (Elegant, 2007). To whatever extent, one thing is certain: Generation Y, or the post-80s generation, is the fastest-growing segment of the workforce. It is important to investigate how this generation differs from more traditional generations in how they think about business and how people should lead them.

Another issue requiring attention in this study concerns the influence of culture on leadership. Cultural assumptions and values about the nature and function of power and authority determine what is feasible for the leaders (Hofstede, 1991). The structuring of relationships, styles of interpersonal interaction, desirable and efficacious leader and subordinate 
characteristics, and the leadership situation, all entail different conceptualizations and enactments of any role akin to leadership (England \& Lee, 1974). Culture for leadership is what water is for fish. It may not be overtly obvious, but it is the element that defines employees' existence, selfawareness, and relationship with others (Hofstede, 2001). Culture is crucial to understand leadership (Hofstede, 1991). Differences in culture could influence the emergence and effectiveness of leaders. Managerial practices and behaviors that are legitimate and acceptable in one culture (or time period) may not be in another. Successful leaders often employ leadership styles consistent with the society's cultural values (Jackofsky, et al., 1988). Thus, the current study adopts Hofstede's $(1991,2001)$ culture value dimensions and identifies the role of these dimensions in shaping leadership.

To organize the proposal, this review of literature contains two parts. In the first part, the theoretical foundations of paternalistic leadership, transformational leadership, trust, harmony, culture, and the effectiveness of leadership are reviewed and discussed. The second part describes the justification of the chosen theoretical framework and development of the hypotheses for later data analysis.

\section{Paternalistic Leadership}

The Origin and Cultural Roots of Paternalistic Leadership

Paternalism is a prevalent managerial style in Asian, Middle-Eastern and Latin American cultures (Plellegrini \& Scandura, 2008). The study of paternalism as a management concept dates back to the early works of Max Weber. According to Weber (1968), domination is stated to be a critical subject in social operation and it plays a crucial part in human collective action. He proposed that there are three ideal-types of domination that are based on legitimacy: legal 
domination, traditional domination, and charismatic domination. Paternalism is one form of legitimated authority and one of the most elementary types of traditional domination.

Weber (1968) believes that an individual's lifestyle is strongly affected by the relationship between that individual and the social community. Furthermore, the relationship between the individual and the social community is controlled by patriarchal authority, in which the patriarch has the power to determine the individual's rights, obligations, and relationship with the social community. Weber (1947) argued that paternalistic practices would become obsolete as organizations became more bureaucratic and relied on rules and the protection of individual rights. Thus, the rational-legal model of bureaucracy would replace traditional forms of control such as paternalism and the shift from paternalism-based traditional organizations to rationallegal organizations would produce better performance (Weber, 1968).

Studies on paternalism emerging from Asia opposed Weber's purely authoritarian view. Paternalism indicates that managers take a personal interest in employees' off-the-job lives and attempt to promote employees' personal welfare (Gelfand, Erez, \& Aycan, 2007). Recently, researchers claimed that paternalism is never completely vanished from even the most rationallegal organizations and that the benevolent aspect in paternalism may be underappreciated in Western literature (Pearce, 2005; Gelfand, et al., 2007). These researchers argued that benevolent leadership includes providing support, protection, and care to subordinates. In paternalistic cultures, people in authority consider it an obligation to provide protection to those under their care and in exchange expect loyalty and deference (Aycan, Kanungo, Mendonca, Yu, Deller, \& Stahl, 2000). This type of leadership stems from cultural traditions and is still prevalent and effective in many business cultures that are high on paternalistic values. 
In the ethnographic study of families from India, Seymour (1999) reported that children are taught very early that their needs do not come first and that they must submit to the authority of others in order to foster family coherence and harmony. Family members are expected to comply with the decisions of the father (Sinha, 1990). These traditional values extend to Indian organizations, where there are strong norms that define who is expected to communicate with whom. For example, junior employees will not simply approach senior employees (Zaidman \& Brock, 2009). Consequently, leaders maintain strong authority whereas subordinates are expected to accept the leader's values as if they were their own.

Studies conducted by Behrens (2009) found that the roots of traditional values shape acceptable leadership styles in Latin America. Latin America is the cultural heir of Spain. The special friendly familiarity between the politicians from upper class and their servants appears to be deeply engrained in Latin Societies (Pitt-Rivers, 1954). Paternalism is ubiquitous in Latin America, where the personalized relationships are preferred over objectified ones. American managerial approaches rooted in individual self-reliance are unattractive to followers nurtured in cultures where organizational engagement and loyalty is exchanged for protection by the powerful (Behrens, 2009). Corporations will do better by showing an interest and helping employees in the non-work dimension of the lives. Martinez (2003) points out a similar relevance of paternalistic relationships in Mexico and concludes that paternalism fits the Mexican cultural values of respect for hierarchical relations and strong family and personal relationships.

Research results reveal that employees from the Middle East also place a highly positive value on paternalism. In Turkey, the patriarchal nature of the family structure and family norms affect business organizations. Turkey has a rich family tradition of unconditional love and 
acceptance of the young child, which coexists with firmness and strict discipline (Martinez \& Dorfman, 1998). The traditional Turkish business context reflects high power distance and collectivistic values, which place a premium on maintaining relationships and place more emphasis on obligation and loyalty (Hofstede, 2001). Employers are responsible for the employees' welfare such as severance payments, which are paid when an employee is fired or a female employee resigns within one year of getting married and claims that her husband does not want her to work. Paternalistic leadership is an effective leadership style in Turkish organizations.

In addition to Turkey, research from Pacific Asia indicates that paternalistic leadership is a prevalent leadership style in current business organizations in Japan (Uhl-Bien, Tierney, Graen, \& Wakabayashi, 1990), Malaysia (Ansari, Ahmad \& Aafaqi, 2004), and China (Farh \& Cheng, 2000). In the Chinese context, Farh and Cheng (2000) claim that paternalistic leadership could be traced to the cultural traditions of Confucianism and Legalism. Under the influence of Confucianism, the father-son relationship is considered paramount and supersedes all other social relations. By virtue of his role, a father has legitimate authority over his children and all other family members. Meanwhile, the legalist doctrine, which was prevalent in dynastic China, called for the emperor to distrust his ministers and maintain absolute power and control over them through political manipulation (Lau, 1979). The confluence of these two traditions leads to Politicized Confucianism, which legitimizes the superior's absolute power and authority over his inferiors. This tradition later generalized to all forms of hierarchical organizations in Chinese societies in which a leader has the right to maintain strong authority over subordinates, and the subordinates are obligated to obey (Lau, 1979). Confucianism also emphasizes mutuality in social relations: that is, a father should be kind to his children, and the children should show 
respect and filial piety to their father. This reciprocity may take the form of genuine gratitude, personal loyalty, or obedience to and compliance with the superior's requests, even beyond what is normally required in the subordinate role (Cheng, Chou, \& Farh, 2000).

\section{The Existing Model of Paternalistic Leadership}

Research on paternalistic leadership has increasingly flourished within the past forty years. Based on the observations and interviews conducted in a large private enterprise in Taiwan, Silin (1976) found that the leadership concepts and behavioral styles of business managers in Taiwan, which is called paternalistic leadership, were greatly different from those in the West. Silin (1976) describes paternalistic leadership as a type of leadership with features such as didactic leadership, moral leadership, centralized authority, maintaining social distance with subordinates, keeping intentions ill-defined, and implementing control tactics.

Following Silin's (1976) research on a large business in Taiwan, Redding (1990) and Cheng (1995) also adopted an indigenous approach to explore middle- and high-level leadership issues within Chinese family businesses (CFB) throughout Hong Kong, Indonesia, Singapore, and Taiwan. Similar to Silin's (1976) research, Redding (1990) and Cheng (1995) both agree that paternalism is a key element in Chinese capitalism. Furthermore, Redding (1990) also suggests that different subordinates have varying degrees of demands for paternalistic leadership. This variance in demanding is because Chinese societies have a strong legacy of personalism, a tendency to allow personal factors to be included in decision-making. Not all subordinates are fit for a benevolent autocratic style. In addition, Cheng (1995) found that paternalistic leadership consists of two broad categories of behavior: shi-en (grant favors) and li-wei (inspire awe or fear). This categorization is consistent with Confucianism, in which a father has legitimate authority over his children and, at the same time, a father should be kind to his children. 
Building on previous works, Westwood (1997) proposed a conceptual model of paternalistic headship for CFBs and suggested that paternalistic leadership is a role that combines discipline and authority with fatherly concern and benevolence. In his conceptual model, Westwood (1997) suggested two basic requirements for paternalistic leadership. One is for order and compliance, and another is social harmony, which is fundamentally embedded in Chinese cultural traditions and their value system. According to Westwood (1997), the behavioral and attitudinal elements of paternalistic leadership include: didactic leadership, nonspecific intentions, reputation building, protection of dominance, political manipulation, patronage and nepotism, conflict diffusion, aloofness and social distance, and dialogue ideal. The core of paternalistic leadership is that it is constituted through a distinctive admixture of clearly delineated and legitimized patriarchal authority, together with a reciprocal obligation for paternal benevolence and virtue.

A more recent depiction of paternalistic leadership is based on two dimensions: behavior and underlying intent (Aycan, 2006). A 2x2 matrix is developed to describe four distinct styles of leadership: benevolent paternalism, exploitative paternalism, authoritarian approach, and authoritative approach. Benevolent paternalism refers to a leader who has a genuine concern for employees' welfare, and employees show loyalty and deference out of respect and appreciation for the employer's care and protection. In exploitative relationships, employees show loyalty and deference mainly because the leaders are capable of fulfilling their needs as well as depriving them from critical resources. The overt leader behavior shared by benevolent and exploitative paternalism is care and nurturance; however, exploitative paternalism is provided solely to elicit employees' compliance to achieve organizational objectives. The difference between authoritarian and authoritative leadership is the underlying intent although both types of 
leadership share control. In authoritarian leadership, employees show conformity to receive rewards or avoid punishment, while in authoritative leadership, employees know that the rules are for their benefit and respect the leader's decisions and willingly comply with the rules (Aycan, 2006).

The domain of paternalistic leadership has mainly been studied by Farh, Cheng, and colleagues (Farh \& Cheng, 2000; Farh, Cheng, Chou, \& Chu, 2006). Their model is widely accepted and used for empirical studies. They defined paternalistic leadership as a style that combines strong discipline and authority with fatherly benevolence and moral integrity couched in a personalistic atmosphere. Based on this definition, paternalistic leadership consists of three important elements: authoritarianism, benevolence, and moral leadership. Authoritarianism refers to leader behaviors that assert authority and control and demand unquestioning obedience from subordinates. The concrete behavior that characterizes authoritarian leadership includes control and domination, underestimating the ability of subordinates, building a lofty image of the leader, and instructing subordinates in a didactic style. Benevolence describes leader behavior that demonstrates individualized, holistic concern for subordinates' personal or familial wellbeing. In return, subordinates feel grateful and obliged to repay when the situation allows. Benevolent leadership goes beyond the work domain and is also applied to personal issues, is long-term oriented, involves the granting of grace and protection to subordinates, and is exercised in the context of strong authority so that the subordinate does not forget who the boss is. Moral leadership is broadly depicted as a leader's behavior that demonstrates superior personal virtues that lead subordinates to respect and identify with the leader. It is characterized by a higher degree of personal integrity, self-cultivation, and selflessness. The actual behavior 
does not abuse authority for personal gain, acts as an exemplar in personal and work conduct, and is upright and responsible.

The vast majority of research on paternalistic leadership focuses on the conceptual model and the outcomes of paternalistic leadership. The development of the antecedents of paternalism is limited. Martinez (2003) conducted field interviews with Mexican managers and identified several antecedent variables, such as employees' respect for social hierarchy, familylike organizational climates, frequent interactions with decision makers, high value for personal relationships, and limited employee decision making. Ansari et al. (2004) identified that leadermember exchange (LMX) is another antecedent variable. They found that in Malaysia, paternalistic leadership is provided only to those who have high-quality exchange relationships with leaders and that paternalistic treatment is contingent on subordinates' task accomplishment. In line with Ansari et al. (2004), Pellegrinin and Scandura (2006) conducted studies in Turkey that confirmed that LMX quality is significantly correlated with paternalistic leadership.

Research on consequences of paternalism has so far been exclusively conducted in field settings. Uhl-Bien et al. (1990) found that in Japan, paternalistic leadership fosters trust among workers and managers, affective motivation versus economic motivation, cooperation throughout the organization, group harmony, and lifetime employee commitment. Researchers also found paternalistic leadership was positively associated with employees' reactivity and obligation (Aycan et al., 2000), loyalty toward leaders (Osland, Franco, \& Osland, 1999: Cheng, Shieh \& Chou, 2002), satisfaction with the leader, commitment to the team (Cheng, Huang, \& Chou, 2002), trust in leaders, organizational citizenship behavior (Cheng et al., 2002b), identification, compliance without dissent, and gratitude (Cheng, Chou, Wu, Huang, \& Farh, 2004). In addition to outcomes related to employees' work attitudes and behavior, the organizational level as well 
as management level outcomes include a decrease of turnover intentions (Cheng et al., 2002a), reduced costs and increased control (Padavic \& Earnest, 1994), and increased job performance (Chou, Cheng, \& Jen, 2005).

The relationship between paternalistic leadership and outcomes is also found to be influenced by some moderator variables. For example, Cheng et al, (2004) found that for subordinates high in traditionality, authoritarianism had a positive relationship with identification, compliance without dissent, and gratitude, whereas for those low in traditionality, authoritarianism was negatively correlated with these outcome variables. Subordinate dependence is another moderator examined by researchers. Farh et al. (2006) found that authoritarianism had a stronger positive effect on fear of supervisor when subordinate dependence was high than when it was low. The results of the study conducted by Cheng (1995) also reveal that authoritarian leadership was negatively related to loyalty to supervisor, organizational citizenship behavior, and job performance when level of job dependence was low, whereas the relationships were slightly positive for high levels of subordinate dependence.

Elaborations of paternalistic leadership

As the three-domain (authoritarianism, benevolence, and morality) model proposed by Farh, Cheng, and colleagues (Farh \& Cheng, 2000; Farh et al., 2006) has been gradually used in empirical studies, researchers report concerns on the negative inter-dimensional correlations among the three domains of paternalistic leadership (Pellegrini \& Scandura, 2008; Chou et al., 2005).

Authoritarianism is found to be negatively correlated with the other two dimensions, benevolence and morality (Cheng et al., 2002b). Authoritarianism not only negatively correlates with the other two dimensions, it also is negatively associated with subordinate outcomes, such 
as team members' commitment to and satisfaction with team leader (Cheng et al., 2002a), loyalty and trust toward leaders, and organizational citizenship behavior (Cheng et al., 2002b). In contrast, benevolence and morality were found to be positively related with each other and with the outcomes such as subordinates' identification and gratitude toward leaders (Cheng et al., 2004).

Researchers (Aycan, 2006; Cheng et al., 2002b) argue that paternalistic leadership is not a unified construct and suggests an alternative method that the scales should be used separately. According to Aycan (2006), paternalistic leadership should be segmented into four separate leadership styles based on two dimensions: the intent of the leader toward the subordinate (either benevolence or exploitation) and the behavioral occurrence of the leader in leading the subordinate (either care or control). Aycan's approach could be problematic as introducing intent into a leadership behavior theory may make the theory hard to verify or falsify empirically. In most cases, unless we ask the leader himself//herself for the intention, there is no way we could observe or ask other people's opinion to get the answer.

In this study, I intend to reconstruct the dimension of authoritarian leadership as authoritative leadership to achieve a positive alignment and coherence among the three dimensions. As demonstrating authority does not necessarily conflict with benevolent and moral leadership behavior, the new conceptualization could help to purge the negative correlations and be better consistent with the theoretical underpinnings of paternalistic leadership.

Part of the negative correlations of authoritarianism with outcome variables and with the other two dimensions may lie in the negative conceptions of the construct originally developed by Western scholars. The term authoritarian carries a negative connotation in the English language whereas "authoritative" does not. In Western management literature, the term, 
authoritarian management style is closely associated with the outdated Theory $\mathrm{X}$, which assumes that humans inherently dislike responsibility and that an average employee wants to be directed (Pellegrini \& Scandura, 2008). Thus, the authoritarian leadership style implies oppression, control and exploitation, and severe punishment of subordinates (Aycan, 2006). Authoritative leadership is defined as leader behaviors that rely primarily on legitimate authority and professional expertise to influence subordinates (Aycan, 2006).

In the Chinese language, the term used to describe the directive or the commanding aspect of leadership in organizations is called "Wei Quan or Quan Wei leadership." The phrase is more neutral without those negative associations of authoritarianism, and more in line with the definition of authoritative leadership. Therefore, the English equivalent of the Chinese term is better for authoritative leadership than authoritarian leadership.

In this study, I adopt the term, authoritative leadership, instead of authoritarian leadership based on two reasons. First, the meanings and connotations of authoritative leadership are more consistent with the indigenous perspectives of high power distance cultures including China (Hofstede, 2001). Chinese of lower status are accustomed to defer or submit themselves to the authority of their superiors, especially when their superiors are perceived to have legitimate authority. Second, a close examination of the original concepts of paternalistic leadership suggests that authoritarianism could well be a misnomer for authoritative leadership. In both Silin's (1976) and Redding's (1990) works, a typical executive in Chinese family businesses not only possesses strong legitimate authority, but also possesses strong technical expertise and business acumen. In view of these considerations, authoritative leadership will replace authoritarian leadership in the measure of paternalistic leadership. 


\section{Transformational Leadership}

Theoretical Framework of Transformational Leadership

Transformational leadership theory has attracted a significant amount of scholarly

attention from across disciplines. This theory originated with the work of Burns (1978) and later was developed by Bass $(1985,1998)$ and others (Avolio \& Bass, 1988; Tichy \& Devanna, 1986; Antonoakis \& House, 2002). The major premise of transformational leadership theory is the leader's ability to motivate followers to accomplish more than they planned to accomplish (Krishnan, 2005). Transformational leaders inspire followers to transcend their own selfinterests for the good of the organization and are capable of having a profound and extraordinary effect on their followers (Judge \& Piccolo, 2004).

Burns (1978) postulated that transformational leaders inspire followers to accomplish more by concentrating on the follower's values and helping the follower align these values with the values of the organization. Furthermore, Burns (1978) identified transformational leadership as a relationship in which the leader and the follower motivate each other to higher levels of interaction, which results in value system congruence between the leader and the follower (Krishnan, 2002).

In 1985, Bass further developed Burns' original formulation of transformational leadership making it more operational. Bass and Avolio (1998) created a set of five categories based on Bass' (1985) work to characterize a transformational leader: 1) idealized influence or attributed charisma, 2) idealized influence or behavioral charisma, 3) inspirational motivation, 4) intellectual stimulation, and 5) individualized consideration (Antonakis \& House, 2002). Idealized influence or attributed charisma is the emotional component of leaders' behavior that moves followers from their self-interest to a major purpose. Idealized influence or behavioral charisma is the leader's sense of mission that drives the ethics and morals of the followers. 
Inspirational motivation is an intangible behavior that expresses confidence to reach the unreachable. Intellectual stimulation is what makes leaders challenge the status quo and influences the intellect of the followers. Lastly, individualized consideration ensures that leaders become coaches and counselors to their followers (Bryant, 2003).

There is a great deal of consensus among researchers on Bass' model of transformational leadership. Based on a literature review, Podsakoff et al. (1990) summarized six key behaviors associated with transformational leaders: 1) identifying and articulating a vision; 2) providing an appropriate model; 3) fostering the acceptance of group goals; 4) expressing high performance expectations; 5) providing individualized support; and 6) expressing intellectual stimulation. In other research, Rafferty and Griffin (2004) examined the theoretical model developed by Bass. They conducted a series of confirmatory factor analysis models on the responses of 1236 individuals and identified five aspects of transformational leadership: vision, inspirational communication, intellectual stimulation, supportive leadership and personal recognition. It is clear that over the last three or four decades, there has been substantial agreement on what transformational leadership.

In addition, Bass (1985) suggested that transformational leadership should supplement or augment transactional leadership, such that transformational leadership should be positively associated with higher levels of subordinate motivation, effort, satisfaction, and performance above those associated with transactional leadership alone. That is, transformational leadership should account for unique incremental variance in outcome variables over that accounted for by the effect of transactional leadership. Transformational and transactional leadership complement each other and both potentially can be displayed by the same leaders (Bass, 1985; Waldman, Bass \& Yammarino, 2000), but that does not mean they are equally important. Transformational 
leadership builds on transactional leadership and produces levels of follower effort and performance that goes beyond what would occur with a transactional approach alone (Judge \& Piccolo, 2004).

Bycio, Hackett, and Allen (1995) found that contingent reward (a transactional behavior) is a basic element of the employment contract that is necessary for the maintenance of minimally acceptable performance. Moreover, if appropriate rewards were combined with an inspirational, challenging work environment emanating from transformational leadership, there was less likelihood that employees would leave the organization. The augmentation effect also is supported by other researchers. For example, Waldman et al. (2000) suggested that the charisma of a leader augments contingent-reward behavior in relation to leadership effectiveness. Contingent reward creates a chance of contractual agreement encouraging subordinates to put forth effort. Thus, contingent-reward behavior can be viewed as the basis of effective leadership, and charismatic leadership can be viewed as adding to that base for greater leader effectiveness. Furthermore, results in a meta-analysis study also revealed that both transformational and transactional leadership behaviors explain a significant amount of unique variance in performance outcomes (Bruke, Stagl, Klein, Goodwin, Salas, \& Halpin, 2006).

\section{Outcomes of Transformational Leadership}

Transformational leaders encompass role modeling, handling of personal relationships with subordinates, duty orientation of the manager, and critical thinking (Ramachandran \& Krishnan, 2009). They seek to raise followers' awareness of the significance of designated outcomes and get them to transcend their self-interests for the good of the organization (Bass, 1985). Overall, transformational leadership provokes a dual effect on both personal and organizational outcomes. 
At the individual level, evidence reveals that transformational leadership is positively related to the subordinate's effort, job satisfaction, and the subordinate's organizational commitment (Ramachandran \& Krishnan, 2009). Researchers also demonstrated that transformational leadership has a positive influence on empowerment (Dvir et al., 2002; Lok \& Crawford, 2004), trust (Podsakoff et al., 1990; Zeffane \& Connell, 2003), self-efficacy beliefs (Tichy \& Devanna, 1986), and motivation (Bass, 1985; House \& Shamir, 1993).

Bass (1985) declared that transformational leaders inspire their followers to go above and beyond their own self-interests for the sake of the organization as a whole. As a result, these leaders are able to bring a deeper insight and appreciation of input received from each member. Bass (1985) further argued that transformational leaders encourage followers to think critically and look for new approaches to do their jobs. When this challenge is given to followers, it motivates them to become more involved in their tasks, which results in an increase in the degree of satisfaction with their work and commitment to the organization.

Transformational leaders construct a participative climate and empowered condition that allows followers to respond quickly and with flexibility to changes in organizational and environmental demands (Ramachandran \& Krishnan, 2009). Trust is an essential element in the relationship that transformational leaders have with their followers (Podsakoff et al., 1990). The degree of trust that exists in an organization can determine much of the organization's character, influence organizational structure, control mechanisms, job satisfaction, job design, commitment, communication, and organizational citizenship behavior (Zeffane \& Connell, 2003).

Research results reveal that self-efficacy is positively related to transformational leadership as well (Waldman \& Spangler, 1989). An increase in confidence and valence of 
outcomes can produce a noticeable rise in followers' efforts to succeed, thus making leadership the stimulus to create effort beyond expectations (Bass, 1985; Tichy \& Devanna, 1986).

Transformational leadership theory emphasizes the role of empowerment as a central mechanism of building commitment to the organization's objectives (Bass \& Steidlmeier, 1999). Dvir, Eden, Avolio, and Shamir (2002) also found that transformational leaders have a direct impact on followers' empowerment, morality, and motivation. Empowered employees are more likely to reciprocate with higher levels of commitment to their organization (Ramachandran \& Krishnan, 2009; Lok \& Crawford, 2004).

By showing respect and confidence in their followers, transformational leaders are able to bring a high degree of trust and loyalty on the part of followers to the extent that followers are willing to identify with the leader and the organization. As a result, followers trust in and emotionally identify with the leader, such that they are willing to stay with the organization-even under very difficult circumstances.

At the organizational level, researchers have shown that transformational leaders affect organizational outcomes. The outcomes include organizational citizenship behavior/performance (Zeffane \& Connell, 2003), organizational culture (Tucker \& Russell, 2004), organizational vision (Hersey \& Blanchard, 1996; Kouzes \& Posner, 1987), organizational change (Yu, Leithwood, \& Jantzi, 2002), and organizational learning and innovations (Tse \& Mitchell, 2010).

Transformational leaders are assumed to "stimulate followers to perform beyond the level of expectations" (Bass, 1985, p.32). Therefore, it seems likely that transformational leaders, by stimulating followers' organizational citizenship behavior (Podsakoff et al., 1990), enhance the quality and quantity of follower performance (Zeffance \& Connell, 2003). 
Several studies also were conducted (Goodwin, Wofford, \& Boyd, 1999; Zaccaro \& Banks, 2001) that demonstrate a positive relationship between transformational leaders and organizational vision. The primary goal of these leaders is to change the current structure of the organization and inspire organizational employees to believe in a new vision that has new opportunities for the individual and the organization as a whole (Tucker \& Russell, 2004).

In an organization, the leader's beliefs, values, and assumptions shape the culture of the organization and these beliefs, values, and assumptions are then taught to other members of the organization (Hersey \& Blanchard, 1996). Transformational leaders influence the organization's culture by interacting with subordinates and helping them discover who they are and what part they play in helping the organization achieve its mission.

Transformational leaders also influence the organization's culture through its impact on organizational productivity. When the values and the culture of an organization are accentuated by transformational leaders, productivity and innovation within the organization improves (Niehoff, Enz, \& Grover, 1990).

Studies also reveal that transformational leadership influences organizational performance positively through organizational learning and organizational innovation. Organizational learning influences organizational performance both directly and indirectly through organizational innovation (Tse \& Mitchell, 2010; Matzler, Schwarz, Deutinger \& Harms, 2008). Moreover, transformational leaders influence organizational culture by helping organizations see the world in different ways (Mink, 1992). As the external environment of the organization changes, transformational leaders influence organizational culture by helping organizations adapt to this new environment (Yu, Leithwood, \& Jantzi, 2002). 
Transformational leadership in China

Redding (1990) has pointed out that managerial leadership among Chinese is primarily transactional, not transformational. Since transformational leadership is more contemporary than traditional transactional theories of leadership, one might expect it to differ sharply from traditional theories of Chinese leadership, such as paternalistic leadership. Yet, transformational leadership may turn out to be more compatible with them than expected. Three dimensions, individualized consideration, high performance emphasis, and modeling, which are extracted from transformational leadership, can be viewed as corresponding to benevolent leadership, authoritative leadership, and moral leadership, respectively.

Individualized consideration indicates that the leader respects followers and is concerned about their personal feelings and needs (Podsakoff et al., 1990). It is similar to the individualized care of benevolent leadership, although some differences remain. The differences include: 1) benevolent behavior not only refers to job related activities but also extends to a subordinate's personal life; 2) benevolent behavior is long-term oriented that includes behaviors of both forgiving and protection; 3) benevolent leadership is enacted in a situation with a large difference in authority and power distance between superiors and inferiors. It is quite different from the Western tradition where equal treatment and equivalent rights are displayed between the superior and subordinate.

A high performance emphasis denotes the behavior that demonstrates the leader's expectations for excellence, quality, and/or high performance on the part of followers (Podsakoff et al., 1990). This aspect of transformational leadership is similar to rigorousness and doctrine in authoritative leadership. An authoritative leader normally is an expert and will try to promote a subordinate's performance to a high level via personal preferences and influences. The third dimension of transformational leadership, modeling, is comparable to moral leadership, which 
implies that leaders set an example for employees to follow that is consistent with the values the leader espouses (Podsakoff et al., 1990). Modeling through transformational leadership stresses that a leader leads by example to set up an integrity paradigm. In contrast, modeling through moral leadership places emphasis on unselfish behavior, including not abusing power for one's own good, and not taking personal revenge in the name of public interest.

The overlap with concepts in transformational leadership makes sense. First, in transformational leadership, the leader is clearly the agent of the transformation, whereas the organization and the followers are the target of the transformation. Thus, a transformational leader has authority over followers in the hierarchy as he/she leads them toward the vision for the organization. This presumption fits well with the hierarchical structuring of Chinese leadership. Second, the defining characteristic of transformational leadership is the ability to transcend the individual interests of the followers, uniting them behind the collective interests of the organization. This orientation fits perfectly with the Confucian definition of the sagely King or the superior gentleman (Yang, Peng, \& Lee, 2008), that the leader should be a wise person with superior character and conduct to lead his/her subordinates. This aspect of transformational leadership is also reflected in the paternalistic leadership dimension of moral leadership.

Third, the component of individualized consideration characterizing transformational leadership largely mirrors the Confucian philosophy of benevolence. Transformational leaders respect their followers and are concerned about their personal feelings and needs. This aspect of transformational leadership is reflected in the paternalistic leadership dimension of benevolent leadership.

Lastly, transformational leadership and its core component of charisma define leadership in terms of followers' identification with the leader. Followers' social-emotional loyalty to and 
identification with the leader is the hallmark of effective leadership in the Chinese Confucian tradition. Under paternalistic leadership, loyalty to one's supervisor is very important as it indicates identification and support with the supervisor and being able to actively cooperate and assist with the supervisor's business (Jiang \& Cheng, 2003).

This inference is supported by Javidan and Carl's (2005) research examining the middle and senior managers in Canada and Taiwan. They found a common cluster of three factors of charismatic leadership in both Canada and Taiwan: visionary, symbolizer, and self-sacrificer. The study reveals that Chinese managers are familiar with core features of charismatic leadership as conceived in the West.

Chen and Farh (1999) conducted a study investigating the suitability of transformational leadership principles for businesses in Taiwan using local employees as participants. They found that in light of the relationship orientation of the Chinese cultural tradition, transformational leadership behaviors had a positive effect on subordinates' job performance.

In line with Chen and Farh's (1999) study, Wang and colleagues (2005) found that the level of transformational leadership of the supervisor significantly affected both in-role and extra-role performance of the subordinates. More importantly, they found that the effect of transformational leadership was mediated by the level of LMX quality that measures the loyalty and respect that followers felt toward the leader. Transformational leadership and LMX quality were highly correlated $(\mathrm{r}=.71, \mathrm{p}<.001)$. Their study highlights the theoretical compatibility among transformational leadership, LMX quality, and traditional Chinese leadership.

China usually is treated as a collectivistic oriented country (Hofstede, 2001). Some studies find evidence that transformational leadership is more compatible with collectivistic values (Walumbwa \& Lawler, 2003; Ergeneli, Gohar, \& Temirbekova, 2007). The researchers 
found that, for subordinates who scored higher on collectivism, transformational leadership was more strongly positively related to job satisfaction and organizational commitment and more strongly negatively related to turnover intentions than for those who score lower on collectivism. Thus, it appears that transformational leadership is not merely compatible, but has enriched and elaborated certain aspects of the Confucian leadership philosophy.

Trust

\section{Conceptual Framework of Trust}

The development of trust theory has, to date, focused on a range of levels of analysis from the interpersonal to the inter-organizational. This study mainly focuses on the interpersonal, especially the trust in leadership within organizational contexts. Various definitions of trust have been raised within this focus.

According to Mayer, Davis, and Schoorman (1995), trust is defined as the willingness of a party to be vulnerable to the actions of another party based on the expectation that the other party will perform a particular action important to the trustor, irrespective of the ability to monitor or control the other party. Hall, Blass, Ferris, and Massengale (2004) suggest that trust implies a belief that an individual will not act opportunistically or in a self-serving manner. It is a belief of a congruence of values. Similar to this, Rousseau, Sitkin, Burt, and Camerer (1998) conceptualized trust as a psychological state compromising the intention to accept vulnerability based upon positive expectations of the intentions or behavior of another.

Some researchers argue that trust entails the assumption of risks fulfilled (Sheppard \& Sherman, 1998; Johson-Geroge \& Swap, 1982). Risk, or having something invested, is requisite to trust, such that trust is evident only in situations where the potential damage from unfulfilled trust is greater than possible gain if trust is fulfilled (Sheppard \& Sherman, 1998; Johnson- 
George \& Swap, 1982). Kee and Knox (1970) also agree that there must be some meaningful incentives at stake and that the trustor must be cognizant of the risk involved. They further argue that accepting the risks is associated with the type and depth of the interdependence inherent in a given relationship.

Different researchers use different components to frame trust. According to McKnight, Cummings, and Chervany (1998), and Currall and Judge (1995), trust consists of two components: trusting intention-one is willing to depend on the other person in a given situation, and trusting beliefs-one believes the other person is benevolent, competent, honest, or predictable in a situation. Dirks and Ferrin (2002) state that interpersonal trust has two dimensions: cognitive trust, which reflects issues such as the reliability, integrity, honesty, and fairness of a referent; and affective trust, which reflects a special relationship with the referent to demonstrate concern about one's welfare.

From another perspective, Whitener, Brodt, Korsgaard, and Werner (1998) viewed trust as an attitude (derived from the trustor's perceptions, beliefs, and attributions about the trustee based upon the trustee's behavior) held by one individual toward another. They propose three facets of trust: 1) trust in another party reflects an expectation or belief that the other party will act benevolently; 2) trust involves a willingness to be vulnerable and risk that the other party may not fulfill the expectations; 3) trust involves some level of dependency on the other party so that the outcomes of one individual are influenced by the actions of another.

Trust also is conceptualized as an intervening process through which other important behaviors, attitudes, and relationships are either bolstered or weakened (McKnight et al., 1998). For instance, employees may initially intend to communicate with their leader, but the communication may be limited or end up with a bad result because of a lack of trust. Research 
that has conceptualized trust as a process has primarily done so under the guise of investigating the development of trust (Khodyakov, 2007), as opposed to trust in and of itself.

Trust also may be viewed as an emergent state. Emergent states refer to cognitive, motivational, or affective states that are dynamic and changeable as a function of contextual factors as well as inputs, processes, and outcomes (Marks, Mathieu, \& Zaccaro, 2001). From this perspective, trust is considered as both an input as well as a proximal outcome depending on the context (Marks et al., 2001). As an emergent state, trust depends on past behaviors that are very specific to certain situations and may be developed over time (Coppola, Hiltz, \& Rotter, 2004).

Based on the review, I found that trust has been examined as a relatively unchanging disposition, a process, or an emergent state in the organizational literature. When trust is treated as a disposition, it has been described as an individual difference called propensity to trust (Rotter, 1967; Mayer et al., 1995). As disposition refers to individual characteristics such as having a good attitude, showing confidence, and being dependable, which are generally unaffected by the environment and consequently relatively stable, researchers suggest that every individual has some baseline of trust where the individual is willing to become vulnerable to others (Rotter, 1967).

This study focuses on trust as an emergent state as opposed to the disposition- or processview. There are three primary reasons: 1) Ample evidence exists from both laboratory experiments and field-based research that individuals differ considerably in their general predisposition to trust other people. Although acknowledging the evidence, researchers generally have not evinced much interest in such individual differences (Gurtman, 1992; Sorrentino, Holmes, Hanna, \& Sharp, 1995). 2) In the current study, the intent is to primarily 
address the willingness to accept vulnerability that evolves over the course of a relationship through repeated interactions and a history of reciprocity. The disposition perspective does little to forward this effort. 3) In addition, the current study is designed to examine trust as a potential mediating variable between the relationship of leadership style and effectiveness. The interest is in making the decision to trust in leadership and whether that trust explains why leader style relates to leader effectiveness. The focus is not on the process of trust development, but whether trust occurs in conjunction with a particular leader style.

\section{Types of Trust in Leadership}

Trust is a primary attribute associated with leadership. As Zend (1997) noted, part of the leader's task is to work with people to find and solve problems, but whether leaders gain access to the knowledge and creative thinking they need to solve problems depends on how much people trust them. Lewicki and Wiethoff (1995) proposed three types of trust in leadership relationships: calculus-based trust, knowledge-based trust, and identification-based trust. Some other researchers labeled calculus-based trust as deterrence-based trust (Shapiro, Sheppard \& Cheraskin, 1992; Child, 2001).

Calculus-based trust is grounded in the potential rewards and punishments for not violating or violating the trust (Lewicki \& Wiethoff, 1995). This type of trust contains the most fragile relationships. One violation or inconsistency can destroy the relationship. It is based on fear of reprisal if the trust is violated. Individuals who are in this type of trust relationship do what they say because they fear the consequences from not following through on their obligations. In an organization, the bond that creates this trust lies in the authority held by the leader and the punishment the leader can impose if the subordinates fail to fulfill their job-related obligations. 
Knowledge-based trust describes a dimension of relationships and confidence in the other party (Lewicki \& Wiethoff, 1995). Most organizational relationships are rooted in knowledgebased trust, that is, trust is based on the behavioral predictability that comes from a history of interaction (Shapiro et al., 1992). Compared to calculus-based trust, knowledge-based trust relies more on information. This knowledge develops over time, largely as a function of experience that builds confidence or trustworthiness and predictability. The better the employees know their leader, the more accurately they can predict what their leader will do. This predictability enhances trust. Research reveals that increases of communication and interaction can help to develop this type of trust (Lweicki \& Bunker, 1996). In an organization, most manager-employee relationships are knowledge-based (Shapiro et al, 1992; Child, 2001) as both parties have enough experience working with each other that they know what to expect.

The third type is identification-based trust, which is based upon the ability of the parties to understand the other's wants and needs, and to identify with them (Lewicki \& Wiethoff, 1995). This highest level of trust is achieved when there is an emotional connection between the parties (Child, 2001). It allows one party to act as an agent for the other and substitute for that person in interpersonal transactions. This form of trust exists because the parties understand each other's intentions and appreciate the other's wants and desires. This mutual understanding is developed to the point that each can effectively act for the other. Controls are minimal at this level; one party doesn't need to monitor the other party because there exists unquestioned loyalty (Child, 2001). This is also the type of trust the managers ideally seek in teams.

This study will focus on identification-based trust because this type of trust emphasizes the parties' understanding of each other's intentions and appreciate each other's wants and desires (Levicki \& Wiethoff, 1995). Not like calculus-based trust, which is the type of trust 
found in new manager-employee relationships (Shapiro et al., 1992), identification-based trust occurs in organizations among people who have worked together for long periods of time and have a depth of experience that allow them to know each other inside and out (Child, 2001). What's more, identification-based trust is developed to the point that each can effectively act for the other. Controls are minimal at this level. Leaders do not have to monitor employees because there is unquestioned loyalty (Child 2001; Levicki \& Wiethoff, 1995). This type of trust better matches the definition clarified in the current study that trust implies a belief that an individual will not act opportunistically or in a self-serving manner (Hall et al., 2004). Knowledge-based trust does not entail this meaning either. This type of trust exists when employees have adequate information about their leader to understand the leader well enough to be able to accurately predict the leader's behavior. At the knowledge-based trust level, if employees believe they can adequately explain or understand the leader's apparent violation, employees can accept it, forgive the leader, and move on in the relationship.

\section{Models of Trust in Leadership}

There is a large quantity of work examining trust with respect to leadership differences across organizational levels. Research reveals that trust is not only an antecedent related to many valued performance outcomes (Williams, 2001; Whitener et al., 1998), but also a process that results from collaborative interaction between the leader and subordinates (Dirks \& Ferrin, 2002). For instance, trust is found to have influence on processes such as communication, cooperation, and information sharing (Ferrin, Dirks, \& Shah, 2003; Rempel, Holmes, \& Zanna, 1985), satisfaction with and perceived effectiveness of the leader (Gillespie \& Mann, 2004), organizational citizenship behavior, decreased turnover (Connell, Ferres, \& Travaglione, 2003), and organizational performance (Rich, 1997, Shaw, 1997). 
While acknowledging the importance of trust in leadership across levels and forms, the current study primarily focuses on the trust that exists between a subordinate and his/her leader. Specifically, the trust that flows from the subordinate upward to his/her leader will be emphasized in this study. This focus primarily reflects trust in vertical leadership as this is where most of the researches were conducted as opposed to peer-to-peer leadership. Following this focus, a few of the most prominent models of trust are briefly discussed below.

One of the most well-known and influential models was proposed by Mayer, et al. (1995), who were some of the first to begin to truly define trust as separate from its antecedents. The model examined the antecedents of trust focusing on ability, benevolence, and integrity. The authors also suggested that perceived risk and the trustor's propensity to trust moderated the level of the trust.

Extending the initial work within Mayer et al's (1995) model, Williams (2001) developed a model that examined trust within groups. Williams (2001) delineated trust antecedents into belief and affect-based categories. The belief-based category is similar to Mayer et al's (1995) original three antecedents: ability, benevolence, and integrity. Ability-based trust is relevant to subordinates that must rely on the leader's capability to perform in the manner that is expected or promised. Benevolence-based trust stems from the belief that the leader cares about the subordinates and will thus act in ways that are in the subordinates' best interest. Integrity-based trust is based on perceptions of the leader as honest and forthcoming, such that he/she will uphold his/her promises and commitments and not act immorally or unfairly. Williams' affectbased category includes emotional states as an antecedent, the moderating role of the motivation to trust, and specification of a distal outcome of trust (i.e., cooperation). 
From a different perspective, Whitener, et al. (1998) delineated the antecedents to managerial trustworthy behavior and correspondingly how such managerial behavior influenced employee perceptions of trust. Three characteristics - organizational, relational, and individual have been identified that impact managerial trustworthy behavior. Among the three, the relational portion of the model links leader behavior, such as consistency, integrity, sharing and delegation of control, communication, and concern, to employee trust perceptions. Whitener, et al. further proposed that some moderating variables, such as perceived similarity and competency, task interdependence, and employee propensity, impact the relationship between leader behavior and development of trust with his/her subordinates. More importantly, their study examined the impacts of managerial behavior from a cross-level perspective and analyzed trust from both the manager and employee's perspectives.

A more comprehensive model was the meta-analytic examination of trust in leadership conducted by Dirks and Ferrin (2002). Their framework expanded the study of the antecedents to trust, conceptualization of trust, and its outcomes. They further examined the moderating role of direct versus indirect leadership on the relationship between trust and outcomes. The antecedent variables in the study include: 1) leader action and practices, which were defined with constructs such as perceived organizational support, participative decision making, and unmet expectations; 2) follower attributes, such as propensity to trust, and 3) relationship attributes, such as length of the relationship.

Three classes of outcomes of trust were also delineated in Dirks and Ferrin's (2002) study: behavioral and performance outcomes, job attitudes and intentions, and correlates. Behavioral and performance outcomes refer to organizational citizenship behaviors and job performance. Job attitudes and intentions refer to job satisfaction, organizational commitment, 
intent to quit, goal commitment, and belief in information. Correlates refer to satisfaction with the leader and leader-member exchange.

In lieu of moderating effects, researchers found that trust has mediating impacts in the leadership process (Podsakoff et al., 1990). As stated by Podsakoff et al. (1990), trust usually is associated with positive emotions, which will make employees who trust their leaders exert extra effort and increase intrinsically-based satisfaction.

Similarly, in the cross-culture investigation on leadership in Australia and China, Casimir, Waldman, Bartram and Yang (2006) confirmed the mediating effects of trust in the leader on the relationship between transactional and transformational leadership and the in-role performance of followers. They further found that culture influences the mediating effects of trust on the leadership-performance relationship such that the mediating effect is stronger in Australia than in China.

In view of the above discussion, it is clear that the degree to which subordinates and coworkers trust their leader is a key component of a leader's effectiveness within organizations. Thus, it is important to recognize and examine trust in leadership within the organizational context.

\section{Harmony}

\section{Ancient Meaning of Harmony}

Harmony is a central concept in the Chinese culture. Unfortunately, despite its significance, it is arguably also the most understudied. Although there are many studies revealing the importance of harmony in Chinese leadership, it is hard to find a concrete definition of harmony. I investigate harmony in terms of original concept described in ancient Chinese philosophy. 
The major Chinese traditions - Confucian, Taoist, Legalist, and Buddhist - all valued harmony, in the general sense of getting along as an ultimate value, although they disagreed on how to achieve it. Therefore, there are various views of harmony. In the present study, I shall place emphasis upon the basic Confucian idea and shall treat different theories as different aspects of a Confucian view.

One of the earliest expressions of harmony was proposed by Shi Bo, a pre-Confucian scholar-minister (Lau, 1979). According to Shi Bo, harmony is different from sameness. He further explained that when two things can be conducted smoothly, it is called harmony. For this reason, things come together and flourish (Lau, 1979).

According to Shi Bo, harmony values diversity rather than sameness. A healthy and prosperous organization relies on its diverse components to come together to reach its goal. This idea is similar to good cooking or good music making: Different ingredients such as sugar and salt mix together to make a good dish. Different instruments do not conflict with each other, rather, they combine together to make a good melody. A healthy family must consist of spouses from different tribes, a prosperous nation must seek wealth from various sources, and a good organization must have managers capable of holding different opinions.

Harmony, as understood in Confucianism, can occur at various levels (Wah, 2009). It can take place within the individual. A person can harmonize various parts of his or her body, the mind-heart, and various pursuits in life into a well-functioning, organic whole (Lau, 1979). Harmony also can take place between individuals at the level of the family, the community, the nation, and the world (Myers, 1989).

Confucianism puts tremendous weight on interpersonal harmony (Wah, 2009). According to Confucianism, there are five fundamental relationships: emperor-subject, father-son, husband- 
wife, elder-younger, and friend-friend. These harmonious relationships are highly valued in

Confucianism. As Mencius, another famous Chinese philosopher, comments, good timing is not as good as being advantageously situated, and being advantageously situated is not as good as having harmonious people (Tsai, 1991). For the Confucianists, harmony among people is, therefore, the most important thing in human affairs.

In view of the Confucian views, the notion of harmony has four characteristics. First, harmony is a meta-physical as well as an ethical notion (Lau, 1979). It describes both how the world at large operates and how human beings ought to act. Second, harmony is by its very nature, relational. It presupposes the coexistence of multiple parties (Lau, 1979). As far as harmony is concerned, these parties possess more or less equal significance. Persons of harmonious mentality see situations, and make judgments on these things, in relation, in context, not in isolation or separation.

Third, harmony by no means implies perfect agreement. In a harmonious circumstance coexisting parties must be in some way different from one another. Harmony is sustained by energy generated through the interaction of different elements in creative tension. Therefore, even unfriendly parties can coexist in harmony. Lastly, the requirement of harmony places a constraint on each party in interaction, and at the same time, provides a context for each party to have optimal space to flourish (Wah, 2009). No one thing can claim absolute superiority over another. Parties in a harmonious relationship are both the condition for and the constraint against one another's growth.

\section{My Definition of Harmony}

Based on the understanding of the literature and Confucian view, I interpret harmony as a state of equilibrium where side by side, all things develop without doing harm to one another, 
and finally lead to an optimal result. This definition is developed under three assumptions: order, relationship, and execution.

First of all, order refers to the institutional order. As mentioned earlier, Confucianism proposes five fundamental relationships in human society: between ruler and minister, parent and child, husband and wife, between siblings, and between friends. There is a clear line between the two parties (e.g., ruler and minister, etc.) in each pair of relationships. Similarly, in an organization, the boss is the boss and the employee is the employee. Everyone in an organization has an appropriate position. The order is relatively constant and does not change with time. According to Confucian thinking, order is not pre-set or fixed, rather, an organization has to generate an order of its own. Only in order, can real harmony be achieved.

Relationship refers to the concrete rules specifying the right relations with one another. Institutions only can be justified when they provide rules of right conduct. In this sense, order is the manifestation of relationship and it, therefore, has relationship as its substance. That is, order is actually supported by relationship and easier to be observed. The rights and responsibilities are well understood and accepted by the employees as well as managers in an organization. There are rules or strong norms that define who is expected to communicate with whom and perform what type of job. Harmony is partly accomplished through following the rules of proper behavior within the status hierarchy (Westwood, 1997). Employees follow these rules or norms and do not simply approach people or jobs beyond their role obligation.

Then, how can we achieve such an order? How might we discover and follow the right way to make the concrete rules? To answer these questions, we must move to the third dimension of harmony, execution. Execution involves a formal condition of the search for right. It is the will-to-right. Leaders discover and follow the right way to make the concrete rules. 
Researchers (Lau, 1979, Wah, 2009) found that harmony was probably used more frequently as a verb (i.e., harmonize) than a noun (i.e., harmony). In other words, people are more interested in determing how to harmonize the relationship within an organization. With this understanding, Confucian harmony is not mere agreement without difference; it is not meant to preserve peace at any cost. Harmony is to make it harmonious; real harmony is a dynamic process.

Confucius did not try to seek true knowledge and nor did he believe that virtue was knowledge. He did not place confidence in perfect knowledge at all, but emphasized the will element of human action as the origin of moral value. An ancient Chinese story may help to understand this. In the story, a son reported to the government that he observed his father stealing a bull from his neighbor. When compared to the father's stealing a bull, Confucius thought the son's behavior of betraying his father was more unacceptable because it violated human nature. Under Confucianism, people are required to show consideration for others in recognition of their humanity and of how their own behavior would be experienced if directed at themselves (Silin, 1976). Thus, researchers (Aycan et al., 2000) found that in some cases, the manager would sacrifice task accomplishment in order to maintain harmonious relationships with and among subordinates. In order to obtain public consensus, the manager attempts private communication and coordination as much as possible. The manager suitably maintains space for suggestions or flexibility to protect mutual dignity and to save face. Thus, unless the private attempt fails, the manager avoids using formal and explicit methods to maintain flexibility.

Execution also involves leaders' behavior of balancing their desires to be lenient with the need to be harsh toward their people (Cheng et al., 2004). When a manager is lenient, the subordinates grow bold. Their boldness must be rectified with harshness. The employees suffer from such ferocity, and due to their suffering, they must be treated with leniency. Leniency 
balances out harshness, and harshness balances leniency. This is the way for most Chinese leaders to achieve harmony.

The three underpinnings - order, relationship, and execution - constitute the main idea of harmony. Therefore, the concept of harmony is herein interpreted as harmony in order, the manifestation of the right relationship, and providing the practical conditions for the actualization of right in organizations (execution).

Relationship between Harmony and Chinese Leadership

Harmony is probably the most cherished ideal in Chinese culture that is associated with Chinese leadership. In the Asian culture, harmony is viewed broadly functional for both internal management and external relations (Gallo, 2008). Internally, this notion helps firms avoid conflict and reduce management costs. Externally, it helps firms establish harmonious environments that are advantageous to the firms. The current study only focuses on the internal harmonious relationship within the organization.

In the theoretical model investigating Chinese leadership, Westwood (1997) argues that harmony is one of the two basic requirements for any leadership situation in the Chinese context. He further claims that such harmony is not based on equalitarian or egalitarian presumptions, nor upon mere exchange values; rather it develops in an environment of clear and acknowledged power distances and inequalities.

According to Westwood (1997), Chinese leadership requiring both order and harmony is distinctive. This dual requirement structure is different from other cultures. For example, because of their femininity orientation (Hofstede, 2001), Scandinavian culture requires forms of social harmony in the workplace, but order and compliance are not required. In the Latin 
American culture, there are some requirements for hierarchical order and autocratic leadership, but the requirements for harmony are not as pronounced.

Westwood's (1997) view complies with Silin's (1976) who argues that harmony occurs not through equality but through the acceptance of socially approved rules of behavior based on ordered hierarchy. A similar argument is also held by Bond and Hwang (1986). According to them, the prerequisites of harmony include individual loyalty and obedience to the authorities. The philosophy of harmony has been translated as keeping harmonious interpersonal relationships (Cheng et al., 2002a), being kind to others (Chou et al., 2005), and smooth cooperation with others (Farh et al., 2006) in the practice of management.

Several researchers reported that harmony is especially precious for cohesion in the organization (Gallo, 2008; Aycan, 2006; Cheng, 1995). They believe that reliance on rule-based management alone is not enough. The manager should consider employees' feelings and respect them. Creating harmony within the organization can help to maintain cohesion in the company; that is, managers must maintain personal communication with all subordinates so as to be aware of problems they are facing, and they should always take action to solve those problems.

The seeking of harmony in the relationships within a company is viewed as one of the Chinese leadership competencies (Cheng et al., 2002b). As leaders are often faced with complex, multi-party negotiations, creating a harmonious result in which all parties are at least reasonably satisfied with the result is important in the Chinese context.

\section{Generation}

Generation refers to a cluster of people born in the same year or in a certain period of time, who share similar values, attitudes, and life styles shaped by the particular epoch, thereby representing the characteristics of that specific era (Rogler, 2002). Different generations have 
different symbols and distinctive characteristics that attach them to the people in the same generation and differentiate them from people in other generations. These common experiences tie a generation's members together by sharing similar values, attitudes and ideas, which can be reflected in the way they view work and the workplace.

In China, some researchers categorize the workforce into four generations (Zhao \& Liu, 2006): Traditional Generation, Zhiqing Generation, Open Generation, and Only-one Generation. The traditional Generation refers to individuals born from 1940 to 1950 . Most of them already have retired. The Zhiqing Generation refers to individuals born from 1950 to 1960, who experienced the Cultural Revolution and the Sent-down Movement (urban or educated people were sent to the countryside to work in the field).

The Open Generation refers to individuals born from 1960 to 1980, who have experienced China's reform process opening to the world. This generation also has experienced educational improvement and economic development in China. Finally, the Only-one Generation refers to individuals born from 1980 to 1990. This is the first generation born after the One Child Policy in China.

As most of people in the Traditional Generation already have retired, and the Zhiqing Generation is also close to retirement age, the Open Generation and Only-one Generation represent the majority in the Chinese workforce. Accordingly, I will focus on these two generations but use the global term "Generation X" and "Generation Y," respectively. Generation X encompasses the people born between 1965 and 1979, whereas Generation Y refers to the group of people born between 1979 and 1994 (Dembo, 2000; Ellen, Kerwin, \& Kerwin, 1999). 
Growing up in the post-Mao 70's and 80's, Generation X in China grew up during the period of Economic Reforms and Openness (Zhang \& Sharitt, 2003). Reforms included the development of a diversified banking system and stock markets. The consumer and export sectors developed rapidly. By the mid-1980s, living standards, life expectancies, literacy rates, and total grain output were up and an urban middle class was growing (Lin, 2001).

Generation $\mathrm{X}$ also grew up with more personal rights and freedoms than the previous generations (Zhang \& Sharitt, 2003). Although Generation X was exposed to many Western elements, they balance between the reinvigoration of China's cultural heritage and exploration of opportunities in the West and remain a strong traditional culture (Dou, Wang, \& Zhou, 2006). Compared with Generation X, Generation Y is characterized as less cynical, more optimistic, more idealistic, more inclined to value tradition, and more similar to Baby Boomers (Wolburg \& Pokrywczynski, 2001). In China, Generation Y is the first generation in the world's history in which a majority of families only have one child due to china's One-Child Policy instituted in 1978 (Ellen et al., 1999). They are also called the "Post-80s Generation" or "Me Generation (Elegant, 2007).”

Generation Y enjoyed the fruits of economic development and never experienced the tough times like their parents did. Many of them were born and brought up in comparatively well off families, received a good education with computers, televisions, the Internet, CD players, and mobile phones as their companions (Stanat, 2005). It is important for both local and foreign organizations in China to learn to manage Generation $\mathrm{Y}$ effectively because they make up about fifty percent of the country's current working-age population (Zhao \& Liu, 2006). 


\section{Culture}

Culture often refers to collectivities in which the members share several psychological commonalities such as assumptions, beliefs, values, interpretations of events, meanings, social identities, and motives, and abide by a set of shared norms in a common manner (Hofstede, 1991). This definition is best suited to the discussion of leadership because it not only covers the conventional societal meaning of culture, but also covers the use of culture in a corporate sense. The word culture is usually reserved for societies which are a social system "characterized by the highest level of self-sufficiency in relation to its environment" (Parsons, 1977, cited by Hofstede, 2001, p. 25). According to Hofstede, although a society may contain different cultural groups, these groups usually share certain cultural traits that make their members recognizable to foreigners as belonging to that society.

The culture-universal perspective argues that there are some universal tendencies in leadership and leadership practices are more similar than different (Bass \& Avolio, 1993, Zagorsek, 2004). Most researchers support the arguments that culture can influence leadership concepts, styles, and practices (Gerstner \& Day, 1994; Hofstede, 2001; House \& Aditya, 1997). This culture-specific perspective claims that although global attitude is a critical skill for leaders to be effective, leaders have to adapt themselves to the cultural environment. Cultures with different characteristics such as language, beliefs, values, religion, and social organizations are generally presumed to necessitate distinct leadership behaviors in different societies. Thus, different leadership prototypes would be expected to occur naturally according to different culture profiles (Hofstede, 1991). Successful leaders often employ leadership styles consistent with society's cultural values (Jackofsky, Slocum, \& McQuaid, 1988).

The most widely recognized culture dimensions are undoubtedly the ones described by Hofstede (1980, 2001). In discussing the relationship between culture dimensions and leadership, 
this study focuses primarily on the five dimensions developed by Hofstede $(1980,2001)$ as these have been studied most extensively to date and are generally presumed to necessitate leadership approaches in different culture clusters (Jackofsky et al., 1988; Hofstede, 2001). The five dimensions are: individualism-collectivism, power distance, uncertainty avoidance, masculinityfemininity, and long-term vs. short-term orientation. These five dimensions together have substantial face-validity and have been empirically demonstrated to be related to many aspects of leadership. These dimensions can be described as follows:

Individualism-collectivism. This dimension describes whether the culture values individuals' goals or group goals (Hofstede, 1980). Individualism and collectivism in a society strongly affect the nature of the relationship between a person and the organization to which he or she belongs. Cultures that are collective exhibit more emotional dependence on the team, and are more conforming, orderly, traditional, team-oriented, and particularistic (Hofstede, 2001). Individualistically oriented cultures value autonomy, self-interest, and performance. In contrast, collective cultures value group harmony, cooperation, and satisfaction (Javidan \& House, 2001). Individualism-collectivism is studied widely in leadership research. For example, Jung and Avolio (1999) found that collectivist values seem to fit well with some of the processes central to transformational leadership, such as the central role of the group and identification processes. They argue that collectivists are expected to be more prone to identify with their leaders' goals and the common purpose or shared vision of the group and organization. Collectivists tend to have a stronger attachment to their organizations and tend to be more willing to subordinate their individual goals to group goals (Triandis, 1995). In contrast, individualists are expected to be more interested in personal goals and motivated to satisfy their 
own self-interests. They may be more motivated by more short-term focused transactional leadership (Jung \& Avolio, 1999).

The characteristic of willing to sacrifice their personal goals for the sake of group goals of collectivists is also related to paternalistic leadership (Triandis, 1995), especially with those elements of collectivism that have to do with hierarchy and submission to group authority and not to elements that emphasize closeness and interpersonal connection (Triandis \& Gelfand, 1988). Triandis and Gelfand (1998) found that collectivists respect traditionalism and authorities. If the leader wants them to act in ways that they believe will benefit the group, they follow the will of leader even when they are not convinced of this course of action or would personally prefer to do something else.

Power distance. This dimension is defined as the degree to which members of a culture expect power to be distributed unequally (Hofstede, 1980). Power distance determines how a community stratifies its individuals and groups with respect to power, authority, prestige, status, wealth, and material possessions (Javidan \& House, 2001). Cultures that are low on power distance tend to be more egalitarian and prefer participatory decision making, while cultures that are high on power distance typically have more layers and the chain of command is felt to be more important (Hofstede, 2001).

Power distance is directly related to leadership. It has an impact on subordinates' expectations and preferences regarding leadership behavior such as whether the leader should provide more guidance, as well as on acceptable or typical patterns of leadership style. People from different cultures associate different characteristics and behaviors with the leadership role and power distance is one of the factors shaping such images of effective leadership (Lord \& Maher, 1991). 
According to Hofstede (2001), in high power distance cultures such as China, there is a centralized decision structure. Subordinates are expected to be told what to do and the hierarchy reflects the existential inequality between higher and lower levels employees. Subordinates are more reluctant to challenge their supervisors and more fearful of expressing disagreement with their leaders (Adsit, London, Crom \& Jones, 1997). As people want and expect more guidance in societies with greater power distance, it has an impact on subordinates' expectations and preferences regarding leadership. Den Hartog and Dickson (1999) suggested that there is a positive relationship between power distance and paternalistic leadership as the culture with high power distance reflects strong family bonds and a sense of fatalism, and an expectation for organizations to take care of their workers as well as the workers' families. On the contrary, however, because the structure emphasizes centralized authority and leadership, stability, and predictability, it creates barriers for the emergence of transformational leaders (Shao \& Webber, 2006). Several studies have demonstrated the negative relationship between power distance and transformational leadership (Shao \& Webber, 2006; Kuchinke, 1999; Smith, Peterson, \& Schwartz, 2002).

Uncertainty avoidance. This dimension indicates whether uncertainty and ambiguity are perceived as threatening within a culture, such that steps are taken to avoid them (Hofstede, 1980). Uncertainty avoidance refers to the extent to which people seek orderliness, consistency, structure, and laws (Javidan \& House, 2001). Low uncertainty avoidance cultures demand less structure and do not display great concern about following rules and procedures. High uncertainty avoidance cultures prefer consistency, structured lifestyles, and clearly articulated expectations (Hofstede, 2001). 
Based on the uncertainty avoidance dimension description, researchers found that low uncertainty avoidance cultures tolerate more innovative, transformational behavior, while high uncertainty avoidance cultures require more transactional leadership (Jung, Bass, \& Sosik, 1995). Shane, Venkataraman, and MacMillan (1995) demonstrated a negative relationship between uncertainty avoidance and the transformational leader role. They suggested that the less uncertainty avoidance, the more people prefer a transformational leader to work through the existing organizational norms, rules, and procedures to achieve goals.

Offermann and Hellmann (1997) looked at actual leader behavior, and found that managers from high uncertainty avoidance countries, compared to those from low uncertainty avoidance countries, tended to be more controlling and less delegating. This phenomenon is reflected in paternalistic leadership, which addresses discipline and authority. The Chinese culture is characterized as high on uncertainty avoidance (Hofstede, 1980), with an emphasis on rules and procedures that may place more demands on leaders than do cultures low in uncertainty avoidance, where there is greater tolerance for ambiguity and innovative behavior (Koopman, Den Hartog, \& Konard, 1999).

Masculinity-femininity. This dimension is the extent to which the dominant values, such as assertiveness, the acquisition of money and objects, heroism, achievement, the quality of life, cooperation, relationships and group decision in a society are "masculine" (Hofstede, 1980). According to Hofstede, masculinity implies dominant values in a society that stress assertiveness and being tough, eager for money and material objects, and not caring for other people or the quality of life. In contrast, values such as warm social relationships, quality of life, and the care of the weak are emphasized in feminine cultures. 
Masculine and feminine cultures create different leader hero types (Hofstede 2001). The heroic manager in masculine cultures is decisive, assertive, and aggressive. In feminine cultures, the hero is less visible, seeks consensus, and is intuitive and cooperative rather than tough and decisive. This "feminine" view of leadership is in line with what is required for harmony; no extreme side should emerge, each party should work side-by-side and smoothly to overcome organizational inertia. According to the description of this dimension, China appears more feministic-oriented reflecting a desire for individuals to use a less direct manner of expressing themselves, where polite and subtle ways of conversing are valued. People keep their emotions in check and more indirect communication is found (Holtgraves, 1997).

Long-term vs. short-term orientation. This dimension deals with a culture's orientation to time - past, present, and future (Hofstede, 1991). According to Hofestede (1991), values associated with long-term orientation are thrift, perseverance and making sacrifices for a longterm goal. They embrace long-term commitments and sacrifice for a purpose (China, Hong Kong, Japan, and South Korea are examples of this orientation). Cultures with a short-term orientation value the past and present resulting in respect for tradition, fulfilling social obligations, and protecting one's face. Efforts should produce quick results. It is important to maintain personal stability and happiness in the present (examples of short-term orientation include United States, Russia, and France).

Long-term vs. short-term orientation also is known as Confucian dynamism (Hofestede, 1991). Long-term orientation links with positive Confucian values such as ordering relationships by status and observing this order, persistence, thrift, and having a sense of shame. Short-term orientation, however, is associated with negative Confucian values such as personal steadiness and stability, respect for tradition, and reciprocation of greetings, favors, and gifts (Hofestede, 
1991). Long-term oriented cultures emphasize every person's commitment to doing their duty in society, thereby contributing to the establishment of social stability and harmony. In these cultures, such as China, not everyone is entitled to speak. A spoken voice in a company is equated with status, and the Confucian notion of hierarchy, role obligation, and codes of behavior are the shared values. These values are consistent with paternalistic leadership.

\section{Leadership Effectiveness}

Leader's effectiveness has been a major concern of leadership researchers and practice for the past several decades (Yukl, 1989; Podsakoff et al., 1990). There were many researchers who tried to solve the mystery of leadership effectiveness in order to provide organizations with the knowledge of how to develop it in their leaders. Over time, researchers examined leadership effectiveness and shed some light on the topic.

Yukl (2006) claims that most authors define leadership effectiveness as the consequences of the leader's actions for followers and other organizational stakeholders. From a different perspective, Bass and Avolio (1994) state that there is a distinction between effective leaders and successful leaders. According to them, successful leaders get their followers to follow them whereas effective leaders motivate and enable their subordinates to reach shared goals. Hogan, Curphy, and Hogan (1994) summarized the literature on leadership effectiveness and organized it in terms of five categories. In the first category, the effectiveness is evaluated in terms of the actual performance of the team or organizational unit. The second category involves using subordinates, peers or supervisors to evaluate leadership effectiveness. In the third category, leadership potential of strangers is evaluated on the basis of their performance in interviews, simulations, assessment centers, or leaderless group discussions. The fourth category states that leadership effectiveness is evaluated using self-ratings. The last category proposes 
that effectiveness is defined by the low end of the continuum-by persons whose careers are in jeopardy or who have derailed.

Leader effectiveness is often measured in terms of the leader's contribution to the quality of group processes as it is perceived by followers and by outside observers (Yukl, 2006). According to Yukl (2006), a follower's perception is one of the indicators and another is the extent to which the leader's organizational unit performs its task successfully and attains its goals. Since people have different values and perceive effectiveness in different ways, there are several factors that need to be considered when examining leadership effectiveness.

Leadership effectiveness is not only perceived differently from one person to another, but also from culture to culture. People from different cultures share the basic concepts but they view things from different perspectives. In some cultures, one might need to take a strong decisive action in order to be seen as an effective leader, whereas in other cultures consultation and a democratic approach may be a prerequisite (Den Hartog, House, Hanger, \& RuizQuintanila, 1999).

For example, researchers have found that Chinese employees rated managers with similar Asian cultural values - Hong Kong, Taiwan and Japanese managers as well as Chinese managers -higher than American managers on leadership effectiveness (Chen \& Tjosvold, 2005). The reason is because Chinese employees are found more tolerant and understanding of Asian managers and they expected Chinese managers to know how best to work with them. Sweetland's (1978) review of managerial productivity concluded that effective leadership and increased group output were a function of the interaction between managers and their subordinates. Hegarty (1974) found that university department chairs who received feedback from subordinates improved their performance. Andersen's (1995) study found a positive 
relationship between high evaluation of a manager's performance and the relationships that the manager had established with his or her subordinates.

In line with these findings, Bernardin and Kiatt (1985) found that managers who were involved in multirater appraisal systems received significantly higher mean effectiveness ratings than those who received no subordinate feedback. McEvoy and Beatty (1989) compared the predictive validity of subordinate evaluations with assessment center ratings and concluded that subordinate ratings were as effective as assessment center data in forecasting managerial performance seven years later.

Whereas some researchers used the leader's supervisor to assess leadership effectiveness (Tsui, 1984), I will ask the leader's subordinates to assess the effectiveness of their leaders. In most organizational contexts, the person typically responsible for evaluating a leader's performance in a formal appraisal process is the leader's superior. Nevertheless, I believe that the leader's subordinates are in the best position to assess the extent to which the leader's behavior is effective or not because they are most likely to see the leader's behavior on a day-to-day basis. In addition, subordinates are often used to assess leader behavior in leadership research (Podsakoff et al., 1990).

\section{Overview of Theoretical Foundations and Hypotheses Development}

Mediating Effect of Trust on the Relationship between Leadership Style and Effectiveness Trust is defined as "a person's expectation, assumptions, or beliefs about the likelihood that another's future actions will be beneficial, favorable, or at least not deterimental to one's interests (Robinson, 1996, p. 576). It is the expectation that arises within an organization of regular, honest and cooperative behavior. Researchers believe that trust is required in a company for the business leader and the others to work well together (Podsakoff et al., 1990; Yukl, 1989). 
There is a sense in the West that people are generally trustworthy. Western leaders usually begin their assignments with a given level of trust in their subordinates. The subordinates maintain their leaders' trust until their leaders are given cause to think differently. Likewise, subordinates are more likely to trust their leaders at first, until the leaders give them a reason to abandon that trust. Compared with westerners, the Chinese have a different understanding of trust.

The Chinese have a more difficult time than Westerners becoming corporate professional managers because of their inclination to deeply trust only people with whom they have a very close relationship (Littrell, 2002). In China, trust is built up over time. As Littrell (2002) explained in his study, the collectivist mentality (which characterizes the Chinese cultures) tends to treat strangers as meaningless objects or as objects to be taken advantage. People whom one does not know are outsiders, to whom one owes no obligations. A collectivist is much less concerned about those who do not belong to the in-group (Casimir et al., 2006). Subordinates in a Chinese business environment need to see the leader's behavior first. They give the leader plenty of respect, but hold back their trust until they see the behavior that backs up their words. Therefore, Chinese leaders need to pay more attention and spend more effort to gain trust from the workforce.

The certainty of trust within hierarchical relationships in organizations has long been recognized (Yukl, 1989; Aarow, 1974; Miller, 1992). As Tyler and Degoey (1996) noted, if organizational authorities have to continually explain and justify their actions, their ability to effectively manage would be greatly diminished. Also, because of the costs and impracticality of monitoring performance, authorities cannot detect and punish every failure to cooperate, nor can they recognize and reward every cooperative act. As a result, efficient organizational performance depends on individuals' feelings of trust toward the organization and their 
willingness to voluntarily defer to organizational authorities. In addition, trust enhances the possibility for individuals to accept dispute resolution procedures and outcomes when there is a conflict. Research has shown that employees are more likely to accept an unfavorable outcome when they trust an authority's motives and intentions (Tyler, 1994).

Podsakoff et al. (1990) examined the impact of transformational leader behaviors on organizational citizenship behaviors, and the study demonstrates a mediating role played by subordinates' trust in that process. Casimir et al. (2006) also found the same mediating effect of trust on the relationship between leadership and performance. Their study is in line with Bennis and Nanus's (1985) study, which suggests that effective leaders are ones that earn the trust of their follower.

Yukl (1989) also identified trust as one of the crucial reasons that followers are motivated by leaders to perform beyond expectations. The leader characteristics most valued by followers are honesty, integrity, and truthfulness (Kouzes \& Posner, 1987). Trust in and loyalty to the leader play a critical role in the leadership model developed by Boal and Bryson (1988).

Chen et al. (2011) examined the relationship between paternalistic leadership and employee inrole and extra-role performance. They found that trust played a mediating role between leader benevolence and morality and both employee in-role performance and organizational citizenship behavior.

The research that was reviewed for this study provides a near unanimous agreement that trust is essential for leadership, regardless what type of leadership is used. Therefore, the following hypotheses are given:

H1: Subordinates' trust in leader partially mediates the relationship between paternalistic leadership and its effectiveness on subordinates. 
H2: Subordinates' trust in leader partially mediates the relationship between transformational leadership and its effectiveness on subordinates.

Mediating Effect of Harmony on the Relationship between Leadership Style and Effectiveness It is widely accepted that managers must keep harmony within an organization to keep it running smoothly and making profits (Cheng, 1995; Cheng et al., 2002b, Gallo, 2008). Internal harmony helps firms avoid conflict and reduce management cost, whereas external harmony helps firms establish harmonious environments that are advantageous to them (Gallo, 2008). For some executives, harmony takes precedence over the firms' profit goals in certain circumstances (Aycan, 2006). Keeping balance and coordination to maintain harmony are important in Chinese organizations. In order to maximize overall benefits, the leader must be willing to make necessary compromises without going against organizational policies in order to gain harmony.

A lot of attention has been devoted to harmony underpinning paternalistic leadership (Farh \& Cheng, 2000; Cheng et al., 2004; Cheng et al, 2002b). Authoritative leadership could be traced to the cultural tradition of Confucianism that in all forms of hierarchical organizations in Chinese societies, a leader has the right to maintain strong authority over subordinates, and the subordinates are obligated to obey. Benevolent leadership also emphasizes the mutual relations between a benevolent ruler and his loyal ministers. The meaning of benevolent leadership corresponds to the relationship dimension of harmony since relationship emphasizes that in an organization, both the employees and the managers accept and are satisfied with the mutual relationship and willing to maintain this relationship. Relational harmony is maintained when each party performs his/her role dutifully. Moral leadership reflects the execution dimension of harmony that not only the leader must appeal to the followers' sense of righteousness, obligation, and goodness, as motivations for action and work, but also the leaders themselves must possess a 
sense of righteousness, obligation, and goodness. Maintaining high level of moral standards is the right way to achieve order and harmonious relationships in an organization.

Harmony is particularly important when firms are facing transition and change under paternalistic leadership (Aycan, 2006). To adapt to the new market economy, some Chinese firms had to lay off employees who did not meet the new requirements. Zhang, Chen, Liu and Liu (2008) interviewed thirty-five business leaders in China and found that the leaders holding the notion that harmony is precious did not just dismiss employees according to the depersonalized downsizing policy. On the contrary, they communicated with these employees and made great efforts, beyond their duties, to solve the employees' problems. For example, they offered the dismissed employees extra financial support, helped them find new jobs, or created some temporary positions for them. These behaviors greatly reduced the negative effects of dismissed employees, diminished the possible harm caused by the dismissed employees, and helped companies to transfer smoothly.

In sum, harmony flourishes even in an environment of clear and acknowledged power distance and inequalities rather than equalitarian or egalitarian presumptions. In the Chinese context, harmony in the workplace is achieved through the culturally-inherent hierarchical ordering, and harmony is maintained by involving concern and considerateness, an element of paternalistic leadership. Based on the above discussion, I hypothesize:

H3: The degree of harmony in the organization partially mediates the relationship between paternalistic leadership and its effectiveness on subordinates.

Prior research reveals that the very notion of transformational leadership exists across cultures (e.g. Javidan \& Carl, 2005; Ergeneli, Gohar, \& Temirbekova, 2007). Different from this opinion, some researchers address the role of cultural values on the relationship between 
transformational leadership and effectiveness (Walumbwa \& Lawler, 2003; Zhang, Chen, Liu \& Liu, 2008).

Organizations that highly value harmony tend to be more collectivistic in nature (Westwood, 1997) - emphasizing the importance of the group rather than their own self-interest. In Chinese traditional cultures, people have a strong identification with in-groups and possible ostracization with out-groups (Casimir et al., 2006). One of the objectives of transformational leadership is to foster group goals, and to promote collaboration and cooperation among group members. Groups with a high degree of harmony have less individualistic viewpoints and thus may have an easier time focusing on group-level goals rather than individual goals. In contrast, those with a low level of harmony are more likely to act according to their own interests rather than for the interests of the collective. Thus, in the Chinese organizations, it is important for transformational leaders to build a harmonious relationship within the organization.

The Western leadership practice of transformational leadership is based on a strong relationship between the leader and the followers. But in the Chinese society, especially as we consider Confucianism, there is a clearly defined distance in this relationship. The five pairs of social roles - between father and son, between the ruler and the subject, between the older and the younger, between husband and wife, and between friends - are understood by all Chinese people. While the power distance between the leader and the followers in China is becoming smaller, the notion of order and span are still the foundation for leaders in a Chinese context. Any attempts to narrow this distance can cause conflict and discomfort (Littrell, 2002).

Therefore, although transformational leadership originated in Western culture and has been demonstrated as a dominant leadership style in the Western business environment, one cannot ignore the impact of harmony even when using transformational leadership in China. 
Leadership behaviors that are consistent with a society's predominant cultural values are evaluated favorably. Thus, I state the following hypotheses:

H4: The degree of harmony in the organization partially mediates the relationship between transformational leadership and its effectiveness on subordinates.

The Moderating Effect of Generation on the Relationship between Leadership Style and Trust Previous research indicates that China's culture is characterized as highly collectivistic (Hofstede, 2001; Lin, 2001); however, recent studies indicate that with the promotion of modernity, individualism values are predominant in the current Chinese culture (Zhang \& Shavitt, 2003), and Generation Y is much more individualistic than collectivistic (Gallo, 2008). One of the characteristics of individualists, according to Casimir et al. (2006), is that they are much less concerned about those who do not belong to the in-group and are, therefore, unlikely to trust outsiders.

According to Casimir et al. (2006), individualist cultures tend to define in-groups based on similar beliefs, attitudes, and values. Although Generation Y takes for granted that a hierarchy exists, they are less willing than earlier generations to accept it (Gallo, 2008). They are less like their parents who have an unquestioning acceptance of hierarchy and who trust authority. Therefore, Generation $\mathrm{Y}$ is more likely to view paternalistic leaders as out-group members. Generation Y draws a line between personal and professional contexts and does not bear this trust outside personal relationships (Elegant, 2007).

Generation X, with collectivistic characteristics, does not draw a very clear line between in-groups and out-groups in an organization. As Casimir et al. (2006) noted, paternalistic leadership practices work better in collectivistic cultures. Traditional Chinese values such as Confucianism influenced this generation as well as the paternalistic leaders. Thus, this 
generation accepts paternalistic leaders easier and trusts in this type of leader more compared with Generation Y.

The core of transformational leadership is to induce followers to act for certain goals that represent the values and the goal of the organization (Bass, 1998), which matches well with an individualist's in-group concept. Because in-group members are expected to be more prone to identify with their leaders' goals and the common purpose or shared vision of the organization. Generation Y, with individualistic values, is more likely to view a transformational leader as an in-group member, and trust in the transformational leader more compared with Generation X. Therefore, I developed the hypothesis as follows:

H5: Generation moderates the relationship between paternalistic leadership and subordinates' trust in leader, such that the positive relationship between paternalistic leadership and trust is stronger for Generation X than for Generation Y.

H6: Generation moderates the relationship between transformational leadership and subordinates' trust in leader, such that the positive relationship between transformational leadership and trust is stronger for Generation Y than for Generation X.

The Moderating Effect of Generation on the Relationship between Leadership Style and Harmony

Traditional Chinese values focus on family, relationships, achievement, endurance, and sacrifice of one's self to the group. They also include the ideal of harmony and hierarchy as the basis for social structure and interaction (Garrott, 1995). Generation X respects authority and hierarchy. These individuals grew up with the belief that loyalty to the leader and institutions would be rewarded; questioning authority is unacceptable. In addition, some paternalistic leadership behaviors, such as meeting followers' needs according to their personal requests, results in providing good care for followers' families, and expresses concern about followers' daily lives. These behaviors reflect strong Confucian ideas and, therefore, greatly satisfy Generation X. Therefore, it is expected that under paternalistic leadership, Generation X will 
have positive feelings about the hierarchical relationships and perceive harmony in the organization.

On the contrary, while Generation Y still holds up traditional values and takes for granted that hierarchy exists, they do not comply with hierarchic rules as does Generation X. Different from previous generations who rarely voice opinions in meetings, Generation $\mathrm{Y}$ is full of confidence in speaking and interacting with their leaders. According to Gallo (2008), for Generation Y, the good boss is the one who stays in the background, teaching through small hints, the one who is highly available to his/her employees and has trust in them, and knows how to share his/her goals and skills with them. Obviously, transformational leaders fit better than paternalistic leaders with Generation Y. Under transformational leaders, Generation Y is expected to perceive a more harmonious relationship than under paternalistic leaders.

Accompanying the profound changes, there has been rapid progress in almost all parts of life, especially in mass media. The Internet also helped Generation Y connect with the outside world (Hung \& $\mathrm{Li}, 2005$ ). As a result of gaining more access to the great amount of information through the Internet, TV, newspaper and other media, Generation $\mathrm{Y}$ has become much better informed, which provides them with more alternatives and choices in life (Dembo, 2000). The mass media also influences their working habits, career pursuits and daily interactions with people in the workplace. Like many youth around the world, Generation $\mathrm{Y}$ has strong advanced technological skills and an urge to be connected globally (Ellen, et al., 1999). As a consequence, they view transformational leadership, a more Western leadership style, more positively than Generation X. Therefore, it is expected that under transformational leaders, Generation Y will recognize less conflict and obtain more positive results than does Generation X. In contrast, it is 
expected that under paternalistic leaders, Generation X will recognize less conflict and obtain more positive results than does Generation Y.

In view of the above discussion, I hypothesize that:

H7: Generation moderates the relationship between paternalistic leadership and harmonious relationship between a leader and his/her subordinates, such that the positive relationship between paternalistic leadership and harmony is stronger for Generation $\mathrm{X}$ than for Generation Y.

H8: Generation moderates the relationship between transformational leadership and harmonious relationship between a leader and his/her subordinates, such that the positive relationship between transformational leadership and harmony is stronger for Generation Y than for Generation X.

\section{Summary}

Although many researchers have recognized the importance of leadership, trust, harmony, generation, culture, and effectiveness, these constructs have not been studied and analyzed as a whole. In addition, leadership in a Chinese context is greatly understudied (Pellegrini \& Scandura, 2008). By linking Western leadership theories and practices with Chinese traditional values, results of this research vill provide a more holistic explanation for the relationships among these constructs. Chinese leaders are seen to function on the basis of meeting the mutually reinforcing dual requirements for legitimized order and compliance, achievement, and the maintenance of social harmony. The cultural basis for meeting these requirements is examined in detail. In addition, this study examines and articulates a culturally informed and warranted ground for a leadership model in the Chinese context.

More specifically, I examine mediating and moderating effects in the leadership paradigm. The mediating effects examined are those of trust and harmony in the relationship between leadership style and effectiveness. The moderating effect examined focuses on how 
different generations impact the relationships between leadership style and trust as well as leadership style and harmony.

The eight hypotheses are developed based on the above relationships. Figure 3 presents the research model for this study. In the next chapter, I will discuss the research methodology for testing the hypothesized relationships in the model.

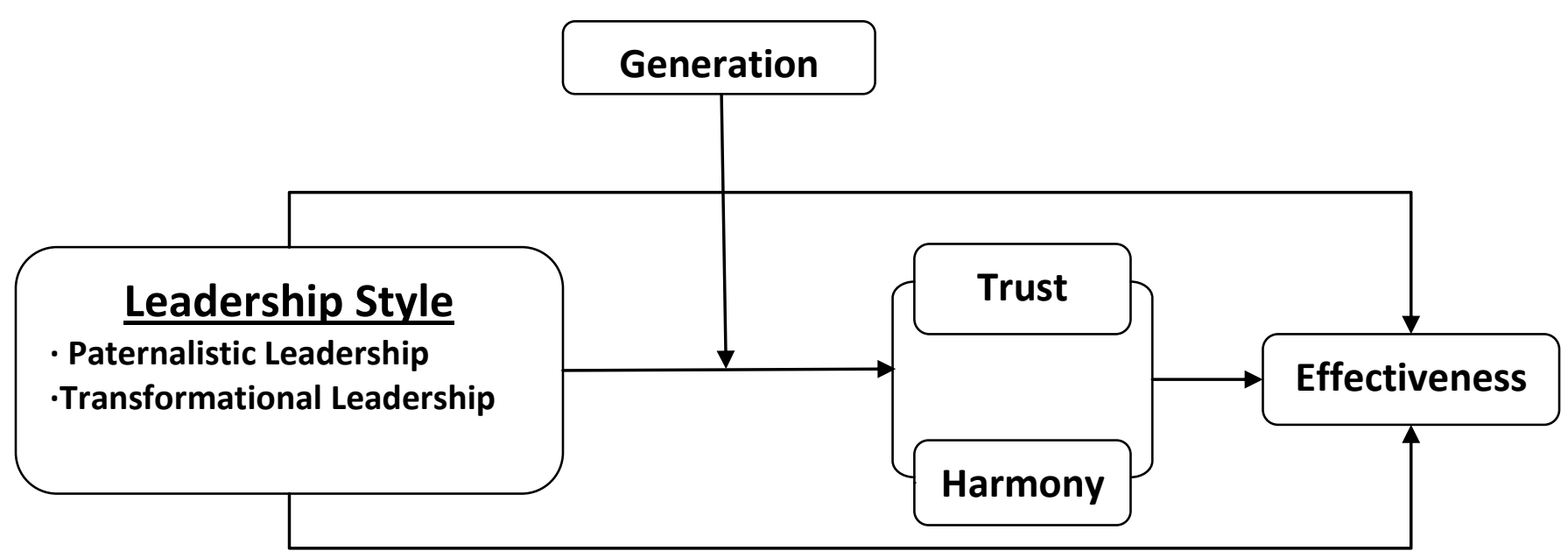

Figure 3. Research Model 


\section{CHAPTER III}

\section{RESEARCH METHODOLOGY}

\section{Introduction}

The primary purpose of this dissertation is to answer the questions whether trust and harmony mediate the relationship between leadership style and effectiveness as well as whether there is a moderator effect of generation on relationships between leadership style and trust and harmony. In Chapter II, a hypothesized model and related hypotheses were developed. Chapter III presents the methodology with which to measure and test the hypotheses. Topics covered in this chapter include the research design, sampling and data collection procedures, the operationalization of constructs, questionnaire design, and pilot study.

\section{Research Design and Data collection}

Research design refers to the structure of an inquiry; it is a logical matter rather than a logistical one (Yin, 1989). When designing research, one must ask, "given a particular research question, what type of evidence is needed to answer the question in a convincing way?" A good research design ensures the evidence obtained answers the research question as unambiguously as possible. A research design is used to structure the research and to show how all of the major parts of the research project, including the sample, measures, and methods of assignment, work together to address the central research questions (Yin, 1989).

Whereas Chapter II focused on the theory building process, Chapter III focuses on the theory testing process in which a statistical model is adopted to test specific hypotheses. The hypotheses will be examined within the context of a field study conducted in China. This method is selected because no manipulation of the constructs/variables is involved. This type of study is characterized as a non-contrived setting without any interference from the researcher. 
Survey research is a method used to gather data from respondents thought to be representative of some population, using an instrument composed of closed structure or open-ended items or questions (Kerlinger \& Lee, 2000). It is a relatively efficient and highly feasible way of collecting information from a large number of respondents and affords faster returns (Gaddis, 1998); therefore, it is considered the dominant form of research design in many social studies (Trochim, 2001). Moreover, survey research is treated as an appropriate mechanism for collecting data to assess interrelationships (Sekaran, 2000). Compared with experimental design, survey research can be used to reach a wide geographic region and collect a wide scope of data. In addition, survey research can provide an efficient means of understanding the vital facts about people, including their beliefs, opinions, attitudes, motivations and behavior (Kerlinger \& Lee, 2000).

Although field survey research is a dominant method used in management related research, it is crucial to realize that internal validity comes before external validity (Cook \& Camphell, 1979). Thus, rigorous development and refinement in the instruments and the design of the study are needed to ensure relevance (Taylor, Goodwin \& Cosier, 2003). Detailed research design procedures are discussed in the following sections.

Sample

Sampling refers to taking a portion of a population or universe as representative of that population or universe (Kerlinger \& Lee, 2000). It is a process of selecting units (e.g., people, groups, organizations) from a population of interest so that by studying the sample one may fairly generalize the results back to the population from which they were chosen (Kerlinger \& Lee, 2000; Trochim, 2001). Both internal and external validity can be strengthened by carefully implementing a sampling practice. 
Hypotheses in this study will be tested by surveying Chinese employees who have worked with their supervisors for more than half a year. Only full-time employees will be used as the subjects because the relationships can only adequately be examined when leaders and followers have had adequate time to formulate opinions and demonstrate behaviors. Selection of organizations includes those organizations providing services and those providing goods. Respondents from either state-owned or private companies, located in different provinces in Mainland China, will be invited to participate in the study. To ensure the procedure is objective, divorced from predilections and biases, the sample will be randomly selected from different cities, work units, age groups, gender, and lengths of time an employee worked for the firm. Demographic information collected will include characteristics of the basic work of the company (service, manufacturing, construction, retail, or wholesale), the number of the full time employees in the company, position, age, gender, education level, the location of the company, whether the company is private or state-owned, and how long the respondent has worked in the company, will be collected. During analyses, however, organization will be used as a control to determine if results differ based on the organization. To reduce potential concern related to evaluating others, participants will be assured that their responses will be kept confidential and used for research only.

Determining the size of the sample is also important for survey research. An appropriate sample size ensures lower sampling error and higher reliability of data, and results can be better replicated when obtaining data from another sample of equal size using the same sampling technique and the same population (Alreck \& Settle, 1985). According to Cohen (1988), power analysis is a major tool that can be used by behavioral scientists to determine the proper sample size. 
The power of a statistical test represents the probability of rejecting the null hypothesis (Cohen \& Cohen, 1983). According to Pedhazur (1997), researchers can seek statistical conclusion validity by trying to reduce both type I and type II errors. Researchers make a type I error when they reject a null hypothesis when it is true. In contrast, they make a type II error when they fail to reject a null hypothesis when it is not true. By keeping the alpha level lower, researchers can reduce the possibility of type I error. Nevertheless, a lower level of alpha might increase the possibility of type II error. In other words, a lower level of alpha might reduce the power of the statistical test to reject the null hypothesis. This dilemma can be overcome by using a sample size that would provide appropriate statistical power to still reject the null hypothesis when it is false.

Researchers always attempt to perform a powerful test of their hypotheses; however, one must be cautious about the level of power used. Too much power might be a detriment to the study, as it might pick up the existence of very insignificant relationships. In other words, with too much power, we might find relationships between variables that might not have any practical value. It is suggested that the chance of committing a type II error ( $\beta$, fail to reject a false null hypothesis), should be held to approximately four times of the chance of a type I error ( $\alpha$, reject true null hypothesis) (Cohen, 1988). As power increases, the chances of a Type II error occurring decrease, but the chances of a Type I error increase. Although there are no formal standards for power, most researchers assess the power of their tests using 0.80 as a standard for adequacy (Cohen, 1988) and I will use .80 as a desirable value for statistical power in this study. This convention implies a four-to-one trade-off between Type I and Type II errors. If $\alpha=.05$ is the probability of a Type I error and $\beta$ (the probability of a Type II error) is four times the chance of 
a Type I error, then $\beta=.20$. The power of the test is $1-\beta=.80$, which meets the standard for adequacy (Cohen, 1988).

Effect size can be measured by looking at the difference between the means of two groups and expressing that difference in terms of standard deviations. It is argued that effect size is a better measure of research outcome than significance level (Kaplan \& George, 1995). The reason is because it is possible to produce statistically significant differences between groups when the size of the effect is in fact very small and this can occur when sample sizes are large (Cohen, 1988). Although a larger sample size is preferred in many social science studies, to achieve adequate power by using the appropriate sample size based on effect sizes is the primary goal here. According to Cohen (1988), a medium effect size represents the average size of observed effects in various fields. For the purpose of this study, a medium effect size of 0.15 is used.

A traditional rule to determine the sample size uses the formula, $\mathrm{n}=\left(\lambda / f^{2}\right)+(k+1)$, where $\mathrm{n}=$ sample size, $\lambda=$ table value for $\alpha=.05, \mathrm{f}^{2}=\mathrm{ES}$ index, $\mathrm{k}=$ number of independent variables (Cohen, 1988). According to this formula, the minimum sample size should be 97 with power of $.80[97=(13.5 / .15)+(6+1)]$. It is suggested that statistics calculated from large samples are more accurate, all things being equal, than those calculated from small samples (Kerlinger \& Lee, 2000). Large samples are not advocated just because large numbers are good in and of themselves. They are advocated in order to give the principle of randomization, a chance to work, to speak somewhat anthropomorphically. With small samples, the probability of selecting deviant samples is greater than with large samples (Kerlinger \& Lee, 2000). Therefore, in this study, I will use another rule of thumb that the proper sample size should be approximately five times the number of items in the questionnaire (Hair, Anderson, Tatham, \& 
Black, 1998). The target number of responses in my study is at least 330 because there are a maximum of 66 items in the questionnaire (before scale purification). I plan to collect more than the minimum data to allow the contingency of missing value, incomplete response, and incorporate data.

\section{Questionnaire Design}

Questionnaires are an efficient data collection mechanism when a study focuses on exactly what is required and has reliable and valid measures of the variables of interest (Sekaran, 2000). The questionnaire includes two sections. The first section is comprised of closed-ended questions that prompt the respondents to give opinions about leadership characteristics of their current managers, assess the degree of trust they have in their leaders, assess the degree of harmonious relationships between managers and themselves, and their perceptions of leadership effectiveness. In the second section of the questionnaires, the demographic information described earlier in the sample description, will be obtained.

The subjects in this study will be sampled from leader-subordinate(s) dyads in Mainland Chinese enterprises across different industries such as steel manufacturing, furniture manufacturing, auto service, and international trade, etc. I will contact the managers in the Chinese businesses for their permission to collect data from their employees. In order to control for manager effect (whether managers should be considered a random or fixed effect), the data will be coded by manager groups; that is, employees working for the same supervisor will be coded as such until it can be determined if it is necessary to control for manager in data analyses. To achieve a high rate of return and ensure accurate coding of questionnaires, I plan to conduct the survey on site and in person. When I cannot personally conduct the survey, I will assign an assistant to substitute for me to distribute the surveys to the subjects. Detailed instructions to the 
questionnaire administrators in both written and verbal forms as well as an envelope will be delivered in advance to ensure the quality of data collection. The assistant will scan and email all the surveys to the researcher after collecting all the completed questionnaires. A cover letter will be included indicating that participation is voluntary, that the responses will be used for research purposes only, and that confidentiality is guaranteed. The cover letter is attached as Appendix I.

\section{Measurement}

The measurement of each construct in the theoretical framework is an integral part of the research design. Stevens (1968) stated that measurement is the assignment of numerals to objects or events in the broadest sense. By looking at the behavioral dimensions, facets, and properties denoted by concepts, researchers can translate them into observable and measureable elements to form an index of measurement of constructs (Sekaran, 2000).

Questionnaires originally written in English were translated into Chinese, and then checked by translation back into English to ensure conceptual consistency. The translation and back-translation were undertaken by bilingual researchers who had studied in both Chinese and English, thus sufficiently educated in both languages as recommended by Bracken and Barona (1991). The original questionnaire was first translated into Chinese by one researcher and translated back into English by another independent researcher as described by Brislin (1970). The translator and back-translator met with the English speaking, monolingual researchers to examine the differences found in the back-translation. After considering their suggestions, some necessary modifications were made, completing the Chinese version of the questionnaire.

The survey instrument developed for this dissertation includes the following constructs that were identified in Chapter II. They are paternalistic leadership, transformational leadership, 
trust, harmony, leadership effectiveness, and generation. Some of the instruments are adopted from existing scales while others are developed by the author based on the literature. All items used in the survey will be measured on a 6-point Likert scale ranging from 1-strongly disagree to 6-strongly agree. An even number of points rather than an odd number is used because Chinese people tend to answer in the middle (Chiu \& Yang, 1987). It is hoped to prevent this response bias by not including a mid-point on the scale. The final set of items is arranged in random order on the questionnaire. The rating scales are randomly flipped and the scales for each construct are attached as Appendix II.

\section{Paternalistic Leadership}

The original three dimensions of paternalistic leadership are authoritarian leadership, benevolent leadership, and moral leadership. Because of the negative correlations obtained between authoritarian leadership and both benevolent and moral leadership, I will revise the subscale to represent a more positive view of leadership, authoritative leadership. Authoritative leadership refers to leader behaviors that rely on legitimate authority and professional expertise to influence subordinates. Benevolent leadership refers to leader behaviors that display personal, overall, and long-term concern for the wellbeing of subordinates. It goes beyond the work domain and is also applied to personal issues and involves the granting of grace and protection to subordinates. It usually is exercised in the context of strong authority so that the subordinate does not forget who is boss (Cheng et al., 2002a). Moral leadership is characterized by a higher degree of personal integrity, self-cultivation, and selflessness. It refers to leader behaviors that conform to social norms and virtues to set an example to others, also a moral leader demonstrates that his/her authority is not only for personal benefit but also for the public good (Westwood, 1997). 
In this study, I will adopt a version of the benevolent and moral leadership scales proposed by Cheng et al. (2000). The internal reliability coefficient was .94 for the benevolent leadership scale and .90 for the moral leadership scale. Farh et al. (2006) verified the reliability in their study also (.87 for benevolent leadership and .86 for moral leadership). Due to the lack of an adequate existing measure, I will construct the items for authoritative leadership starting with the original items in the authoritarian scale. The leader behaviors used to measure authoritarian leadership that have negative implications, such as "make all decisions no matter if they are important or not," and "punish subordinates if they do not follow supervisor's rules to get things done," have been reworded and replaced by "makes final calls on key decisions" and "holds employees accountable for work rules and high performance standards." I will include items for both authoritative and authoritarian leadership to determine if authoritative leadership purges the negative effects of authoritarian leadership as expected.

In previous research, the paternalistic leadership scale was developed to include three distinct dimensions rather than subdomains of an overall construct because of the negative correlation between the authoritarian leadership dimension and the other two dimensions (Pellegrini \& Scandura, 2008). One of the purposes of this study is to purge the negative correlation among the three dimensions by replace authoritarian leadership with authoritative leadership so that the paternalistic leadership scale can be measured as an overall construct. Therefore, the total score of the paternalistic leadership scale is calculated by summing across all 16 items of the three subscales. The questionnaire items for each of the three dimensions of paternalistic leadership are shown in Table 1-a. Questionnaire items for authoritarian leadership are presented in Table 1-b. 
Transformational Leadership

According to Podsakoff et al. (1990), transformational leadership is multidimensional in nature and consists of six key behaviors: identifying and articulating a vision, providing an appropriate model, fostering the acceptance of group goals, promoting high performance expectations, providing individualized support, and encouraging intellectual stimulation. I will use the measure of transformational leadership developed by Podsakoff et al.(1990). The internal consistency reliabilities for all of the dimensions of transformational leadership behavior meet or exceed Nunnally's (1978) recommended level of .70 (e.g., .87 for "core" transformational leader behaviors - three dimensions, identify and articulate a vision, provide an appropriate model, and foster the acceptance of group goals, compose core transformational leadership behavior; .78 for the dimension of high performance expectations; .90 for the dimension of individualized support; and .91 for the dimension of intellectual stimulation). Consistent with previous research, the score for the transformational leadership scale will be calculated by summing the points across all 23 items (Chen \& Farh, 1999; Zhang, Cao, \& Tjosvold, 2010). Zhang et al. verified that Cronbach's alpha for the six dimensions range from .76 to .93, and the alpha for the full scale is 96. The questionnaire items for each of the six dimensions are shown in Table 2. 
Table 1-a. Measures and Questionnaire Items of Paternalistic Leadership

\begin{tabular}{|c|c|}
\hline & Items \\
\hline Authoritative Leadership & $\begin{array}{l}\text { 1. My supervisor knows best what is good for me. } \\
\text { 2. The experience and wisdom of my supervisor are the best guidelines } \\
\text { for me. } \\
\text { 3. My supervisor expects subordinates to comply with and follow } \\
\text { through on directives. } \\
\text { 4. My supervisor makes final calls on key decisions. } \\
\text { 5. My supervisor holds employees accountable for work rules and high } \\
\text { performance standards. }\end{array}$ \\
\hline Benevolent Leadership & $\begin{array}{l}\text { 1. My supervisor handles what is difficult to do or manage in everyday } \\
\text { life for me. } \\
\text { 2. Beyond work relations, my supervisor expresses concern about my } \\
\text { daily life. } \\
\text { 3. My supervisor ordinarily shows a kind concern for my comfort. } \\
\text { 4. My supervisor takes good care of my family members as well. } \\
\text { 5. My supervisor meets my needs according to my personal requests. } \\
\text { 6. My supervisor will help me when I'm in an emergency. }\end{array}$ \\
\hline Moral Leadership & $\begin{array}{l}\text { 1. My supervisor set himself a good role model to follow. } \\
\text { 2. My supervisor does not take advantage of me for personal gain. } \\
\text { 3. My supervisor is an upright and honest person; he/she never } \\
\text { promotes his/her private interests under the guise of serving the } \\
\text { public. } \\
\text { 4. My supervisor never avenges a personal wrong in the name of public } \\
\text { interest when he/she is offended. } \\
\text { 5. When a mistake occurs, my supervisor will take responsibility. }\end{array}$ \\
\hline
\end{tabular}


Table 1-b. Measures and Questionnaire Items of Authoritarian Leadership

\begin{tabular}{|c|c|}
\hline & Items \\
\hline Authoritarian Leadership & $\begin{array}{l}\text { 1. My supervisor asks me to obey his/her instructions completely. } \\
\text { 2. My supervisor always behaves in a commanding fashion in front of } \\
\text { employees. } \\
\text { 3. My supervisor determined all decisions in the organization whether } \\
\text { they are important or not. } \\
\text { 4. In my supervisor's mind, the standard subordinate is an employee } \\
\text { who obeys his commands completely. } \\
\text { 5. We have to follow supervisor's rules to get things done. If not, } \\
\text { he/she punishes us severely. } \\
\text { 6. My supervisor emphasizes that our group must have the best } \\
\text { performance of all the units in the organization. }\end{array}$ \\
\hline
\end{tabular}

Table 2. Measures and Questionnaire Items for Transformational Leadership

\begin{tabular}{|l|l|}
\hline \multirow{2}{*}{ Identify and } & \multicolumn{2}{|c|}{ Items } \\
\cline { 2 - 3 } Articulate a & 1. My supervisor has a clear understanding of where we are going. \\
Vision & 2. My supervisor paints an interesting picture of the future for our group. \\
\hline Provide an & 4. My supervisor is always seeking new opportunities for the organization. \\
Appropriate & 5. My supervisor inspires others with his/her plans for the future. \\
Model & 1. My supervisor leads by "doing", rather than simply by "telling." \\
\hline Fostering the & 2. My supervisor provides a good model for me to follow. \\
Acceptance of & 1. My supervisor leads by example. \\
Group Goals & 2. My supervisor fosters collaboration among work groups. \\
\hline High & 3. My supervisor gets the group to work together for the same goal. \\
Performance & 1. My supervisor develops a team attitude and spirit among employees. \\
Expectations & 2. My supervisor shows us that he/she expects a lot from us. \\
\hline & 3. My supervisor will not settle for second best. \\
Provide & 1. My supervisor acts without considering my feelings. (Reverse scored) \\
Individualized & 2. My supervisor shows respect for my personal feelings. \\
Support & 3. My supervisor behaves in a manner thoughtful of my personal needs. \\
\hline & 4. My supervisor treats me without considering my personal feelings. (Reverse \\
Intellectual & 1. My supervisor challenges me to think about old problems in new ways. \\
Stimulation & 2. My supervisor asks questions that prompt me to think. \\
\hline
\end{tabular}


Trust

The concept of trust used in this study refers to employees' faith in and loyalty to their leaders. The instrument for trust is borrowed from the existing literature (Podsakoff et al., 1990). Cronbach's alpha for trust was .90. Casimir et al. (2006) used the same scale to test the mediation effects of trust on the leadership-performance relation in different culture contexts. The Cronbach's Alpha of trust was .87 for the Australian sample and .85 for the Chinese sample. The scale consists of six items. The scale of trust is scored by summing the points across all six items and the questionnaire is presented in Table 3.

Table 3. Measures and Questionnaire Items of Trust

\begin{tabular}{|l|l|}
\hline \multirow{4}{*}{ Trust } & \multicolumn{2}{|c|}{ Items } \\
\cline { 2 - 3 } & 1. I feel quite confident that my Supervisor will always try to treat me fairly. \\
2. My supervisor would never try to gain an advantage by deceiving workers. \\
3. I have complete faith in the integrity of my supervisor. \\
4. I feel a strong loyalty to my supervisor. \\
5. I would support my supervisor in almost any emergency. \\
6. I have a divided sense of loyalty toward my supervisor. (Reverse scored) \\
\hline
\end{tabular}

Harmony

In Chapter II, harmony is defined as a state of equilibrium where side by side, all things develop without doing harm to one another, and finally lead to an optimal result. Due to the lack of existing measures, I constructed the items for harmony based on the Confucian notion of harmony. The definition of harmony consists of three elements: order, relationship, and execution. Although harmony contains three elements, they are not equally weighted. Order is the prerequisite of relationship, and both order and relationship are prerequisites of execution. Item 1 is developed to measure both order and relationship. Items 4 and 5 are developed to measure execution. Items 2 and 3 are developed to measure execution also, but they are assuming order and relationship both exist. Because of the relationship between the three 
elements of harmony, only a total score will be offered by summing across all five items. The questionnaire items of harmony are shown in Table 4.

Table 4. Measures and Questionnaire Items of Harmony

\begin{tabular}{|l|l|}
\hline \multirow{3}{*}{ Harmony } & \multicolumn{1}{|c|}{ Items } \\
\cline { 2 - 3 } & $\begin{array}{l}\text { 1. Employees behave in accordance with predetermined positions in organization. } \\
\text { 2. In some cases, my supervisor would sacrifice task accomplishment in order to } \\
\text { maintain harmonious relationships with and among subordinates. }\end{array}$ \\
3. $\begin{array}{l}\text { My supervisor has the ability to balance his desire to be lenient with the need to be } \\
\text { harsh toward his people. }\end{array}$ \\
$\begin{array}{l}\text { 4. In complex environment, my supervisor is effective in balancing relationship so } \\
\text { that all parties are reasonably satisfied with the result. }\end{array}$ \\
5. My supervisor values personal relationships.
\end{tabular}

Leadership Effectiveness

Leader effectiveness is often measured in terms of the leader's contribution to the quality of group processes as it is perceived by followers and by outside observers (Yukl, 2006).

According to Yukl (2006), a follower's perception is one of the indicators of leader effectiveness and another is the extent to which the leader's organizational unit performs its task successfully and attains its goals. In this study, I will use subordinates to evaluate leadership effectiveness. Leaders' subordinates are believed to be in the best position to assess the extent to which their leaders' behavior is effective or not as they are most likely to see their leaders' behavior on a day-to-day basis (Hogan, Curphy, \& Hogan, 1994). As this study focuses on the relationships between leaders and their subordinates, the subordinates, as direct recipients of their leaders' behaviors, provide an appropriate source of information on their leadership effectiveness.

Scales for leadership effectiveness were borrowed and adapted from previous research (Chen \& Tjosvold, 2005; Chen \& Farh, 1999). Item 1 is from Chen and Farh (1999) and the remaining four items are from Chen and Tjosvold (2005). Because this scale consists of items pulled from two measures, no reliability data is reported here. A 5-item scale will be used to measure leader 
effectiveness (See Table 5) and the total scale score will be calculated by summing up all the points across five items.

Table 5. Measures and Questionnaire Items of Leadership Effectiveness

\begin{tabular}{|l|l|}
\hline \multirow{4}{*}{ Leadership Effectiveness } & \multicolumn{1}{|c|}{ Items } \\
\cline { 2 - 3 } & $\begin{array}{l}\text { 1. My supervisor performs his/her leader roles appropriately. } \\
\text { 2. My supervisor exercises his/her responsibilities well as a leader. } \\
\text { 3. I am satisfied with my supervisor's overall effectiveness as a } \\
\text { leader. }\end{array}$ \\
4. The way my supervisor manages the work inspires me to a better \\
5. I cab performance
\end{tabular}

Generation

Generation is a categorical variable having two categories: Generation X and Generation Y. Generation X refers to individuals born from 1965 to 1979 . Generation Y refers to individuals born from about 1980 to 1994 . One question in the demographic section of the questionnaire asks about the respondent's age. Those aged 31 or older will be classified as Generation $\mathrm{X}$ and those below age 31 will be categorized as Generation Y.

\section{Control Variables}

To enhance the homogeneity of the sample or to control for some external factors that might affect the relationships being studied, it is important to consider variables that should be controlled. Previous research indicates that individual perception of leadership effectiveness differs by gender, educational level, the nature of company, whether it is state-owned or private, and the geographical location of the company (Farh \& Chen, 2000; Pellegrini \& Scandura, 2008). Therefore, it is important to collect data on these variables and control for them because they may provide alternative explanations for variance. Gender is scored as 1 for male and 2 for female. Educational level includes six levels: $1=$ high school diploma; 2 = vocational/technical 
certificate; 3 = associate certificate; 4 = bachelor's degree; $5=$ master's degree; $6=$ doctoral degree. The nature of company is categorized into private company (scored as 1) and stateowned company (scored as 2). The respondents are asked to fill in the name of the city in which the company is located. All of companies are located in four cities: $1=$ Beijing; 2 = Changsha; 3 = Xiangxiang; 4 = Hengyang.

\section{Reliability and Validity}

To provide meaningful results, measurement instruments should have good reliability and validity. According to Bohrnstedt (1983), it is important to establish the reliability and validity of a measurement instrument by recognizing the importance of knowing whether or not items measure what they are intended to measure (validity) and the degree to which items give a consistent or repeatable result (reliability).

Reliability concerns the extent to which a measure is repeatable or stable over a variety of conditions and circumstances (Nunnally, 1978). It is an evaluation of measurement consistency. In this study, I will report internal consistency reliabilities using Cronbach's alpha. Two different methods can be used to calculate Cronbach's alpha: a variance/covariance matrix or an evaluation of the average correlation among items in a scale (Nunnally, 1978). According to Nunnally, a Cronbach's alpha value of .70 is adequate for internal consistency reliability. In addition, the improvement of Cronbach's alpha will be assessed by retaining or deleting items for each scale, along with the subjective assessment of the importance of the item. Three criterion should be considered when improving Cronbach's alpha: 1) at least three items in each scale should be retained in order to cover the construct adequately; 2) an item should be removed only when it does not greatly affect the overall content of the scale; and 3) if the removal of an item yields only a slightly better reliability, the item should be retained in the scale. 
Another key issue is the construct validity. Construct validity is used to provide evidence that the results obtained from the use of the measure fit the theories around which the test is designed (Sekaran, 2000). The construct validity of measures is a necessary condition for the development and testing of theories (Peter, 1981). The face validity of the instrument can be demonstrated by discussing the instruments with professors and researchers in related areas. Although content validity and face validity are important for a measurement instrument, the most important validity question is whether the test instrument measures the concept it was designed to measure. Consequently, I will assess convergent and discriminant validity of the variables in my study.

Convergent validity assesses the degree to which multiple attempts to measure the same concept are in agreement. It will be tested by studying item-total correlations using confirmatory factor analysis. Convergent validity is evaluated by examining the factor loading within each construct. If the factor loadings are significant $(p<0.01)$, the convergent validity is confirmed. Discriminant validity evaluates the degree to which the measures of different concepts are distinct. It can be examined by comparing the squared correlations between constructs and variance extracted for a construct (Fornell \& Larcker, 1981). If the squared correlations for each construct are less than the variance extracted by their indicators measuring that construct, it indicates that the measure has adequate discriminant validity.

\section{Pilot Study}

A pilot study involves the use of a questionnaire with a small sample size to test logistics and gather information prior to a larger study, in order to improve the latter's quality and efficiency. A pilot study can reveal deficiencies in the design of a proposed research method or procedure and these can then be addressed before time and resources are expended on large scale 
studies. As Backstrom and Hursch (1963) pointed out, before the actual administration of the surveys, a pilot study for contextual refinement should be conducted to ensure the clarity, userfriendliness, wording and meaning of the questionnaire. Particularly because there are two instruments newly developed for this study, pilot testing is necessary. Further, by seeking the expert review, the face and content validity of the questionnaire can be improved.

The primary objective of pilot testing of this study is to test the instrument, including a test for reliability and validity. It is because the questionnaires were translated from English to Chinese. It is important to check the conceptual consistency during the translation. The pilot testing is necessary also because there are two newly developed instruments - harmony and authoritative leadership. Nunnally (1978) concluded that a Cronbach's alpha that meets or exceeds .70 is acceptable for newly developed instruments and this is the primary target intended to achieve in the testing. Although other scales have been studied and validated in previous research, it is still necessary to check whether the questionnaires maintain their reliability and validity in this study after adjustment and modification. Furthermore, administering the pilot test can help to refine the questionnaire and to test procedures based on respondents' comments to achieve content and face validity.

\section{Demographic Characteristics of the Pilot Study Sample}

A small amount of data was collected from four different cities in China. The respondents were notified in a letter that by returning the survey, they were giving their consent to participate in the study (see Appendix I). The sample consists of 67 employees across different industries. After visual screening, one questionnaire response was deleted because all answers were rated as either 6 or 1, opposite extremes in the response scales. This pattern of ratings indicated lack of thought provided when answering and had the tendency to skew results. 
Therefore, the total sample size for the pilot study was 66 . Table 6 summarizes the demographic profile of the employee sample.

Table 6. Demographic Profiles of the Sample

\begin{tabular}{|c|c|c|c|}
\hline Variable & Category & $\mathrm{n}$ & Percentage \\
\hline Gender & $\begin{array}{l}\text { Male } \\
\text { Female }\end{array}$ & $\begin{array}{l}45 \\
21\end{array}$ & $\begin{array}{l}68.2 \\
31.8\end{array}$ \\
\hline Age & $\begin{array}{l}\text { Under } 31 \text { years old } \\
\text { Over } 32 \text { years old }\end{array}$ & $\begin{array}{l}37 \\
29\end{array}$ & $\begin{array}{l}56.1 \\
43.9\end{array}$ \\
\hline Education & $\begin{array}{l}\text { High School } \\
\text { Technical Certificate } \\
\text { 2-year College } \\
\text { Bachelor Degree } \\
\text { Graduate Degree }\end{array}$ & $\begin{array}{l}18 \\
12 \\
14 \\
21 \\
1\end{array}$ & $\begin{array}{l}27.3 \\
18.2 \\
21.2 \\
31.8 \\
1.5\end{array}$ \\
\hline Industry & $\begin{array}{l}\text { Retail } \\
\text { Wholesale } \\
\text { Manufacture } \\
\text { Construction } \\
\text { Service } \\
\text { Other }\end{array}$ & $\begin{array}{l}1 \\
14 \\
10 \\
2 \\
33 \\
6\end{array}$ & $\begin{array}{l}1.5 \\
21.2 \\
15.2 \\
3.0 \\
50.0 \\
9.1\end{array}$ \\
\hline Company Size & $\begin{array}{l}\text { Less than } 10 \text { employees } \\
11-80 \text { employees } \\
100-200 \text { employees } \\
800-1200 \text { employees }\end{array}$ & $\begin{array}{l}5 \\
53 \\
9 \\
2\end{array}$ & $\begin{array}{l}7.6 \\
80.3 \\
9.1 \\
3\end{array}$ \\
\hline Tenure & $\begin{array}{l}1-2 \text { years } \\
3-5 \text { years } \\
6-8 \text { years } \\
10-16 \text { years } \\
\text { More than } 16 \text { years }\end{array}$ & $\begin{array}{l}33 \\
12 \\
4 \\
12 \\
5\end{array}$ & $\begin{array}{l}50.0 \\
18.2 \\
6 \\
18.2 \\
7.6\end{array}$ \\
\hline Nature of Company & $\begin{array}{l}\text { State-owned } \\
\text { Private }\end{array}$ & $\begin{array}{l}25 \\
41\end{array}$ & $\begin{array}{l}62.1 \\
37.9\end{array}$ \\
\hline Position & $\begin{array}{l}\text { Staff } \\
\text { Group Leader } \\
\text { Engineer }\end{array}$ & $\begin{array}{l}55 \\
3 \\
8\end{array}$ & $\begin{array}{l}83.6 \\
4.5 \\
11.9\end{array}$ \\
\hline Geographic Location & $\begin{array}{l}\text { Beijing } \\
\text { Changsha } \\
\text { Xiangxiang } \\
\text { Hengyang }\end{array}$ & $\begin{array}{l}3 \\
35 \\
23 \\
4\end{array}$ & $\begin{array}{l}4.5 \\
54.6 \\
34.8 \\
6.1\end{array}$ \\
\hline
\end{tabular}

Pilot Study Analysis

Before evaluating the relationships among the variables, the descriptive statistics of the data were analyzed. This process included evaluation of the normality, constancy of variance, 
heterogeneity, independence, and standardized residuals, and the results supported the use of regression in the analyses (see Aguinis, Peterson, \& Pierce, 1999). Separate factor analyses were conducted for each construct. For the sake of convergent validity, 0.4 was used as the factor loading cut-off point (Hair Anderson, Tatham, Black, 1998). Tables 7-a and 8 show the factor loadings for paternalistic leadership and transformational leadership separately. Items for paternalistic leadership loaded clearly into three separate dimensions as previous research indicates (Cheng et al., 2000). The Cronbach's alpha for the three dimensions (moral, benevolent and authoritative leadership) ranges from .78 to .87 , and the alpha for the full paternalistic leadership scale is .85. Six separate dimensions were identified for the transformational leadership scale as suggested in previous research (Podsakoff et al., 1990). The Cronbach's alpha for the six dimensions ranges from .73 to .85 , and the alpha for the full transformational leadership scale is .84. All items were retained in both the paternalistic leadership and transformational leadership scales. Authoritarian leadership had one item (Authoritarian leadership 6) that loaded as a second factor and was deleted to ensure a single factor (see Table 7-b). The Cronbach's alpha for authoritarian leadership scale is .84 after item 6 was deleted. All five items of harmony loaded as one component (see Table 9). The Cronbach's alpha for the harmony scale is .75. Trust had one item (Trust 5 , see Table 10) and effectiveness had two items (Effectiveness 2 and 3, see Table 11) that loaded as a second factor. To ensure a single factor for each, Trust 5 and Effectiveness 2 were deleted. Effectiveness 3 was retained because this item also strongly loaded in the first factor (.60). The Cronbach's alphas are .80 for trust and .74 for effectiveness after items were deleted. 
Table 7-a. Rotated Component Matrix ${ }^{\mathrm{a}}$ of Paternalistic Leadership

\begin{tabular}{|l|r|r|r|}
\hline & \multicolumn{3}{|c|}{ Component } \\
\cline { 2 - 4 } & 1 & 2 & \multicolumn{1}{|c|}{3} \\
\hline Moral Leadership1 & .852 & .163 & -.044 \\
Moral Leadership2 & .764 & .292 & .013 \\
Moral Leadership4 & .755 & .192 & .157 \\
Moral Leadership5 & .640 & .254 & .337 \\
Moral Leadership3 & .635 & .540 & .084 \\
Benevolent Leadership2 & .268 & .748 & .162 \\
Benevolent Leadership6 & .303 & .740 & .092 \\
Benevolent Leadership4 & -.285 & .713 & .411 \\
Benevolent Leadership1 & .377 & .660 & .339 \\
Benevolent Leadership5 & .337 & .650 & .050 \\
Benevolent Leadeership3 & .380 & .586 & .222 \\
Authoritative Leadership4 & -.199 & .037 & .835 \\
Authoritative Leadership3 & .080 & .111 & .753 \\
Authoritative Leadership5 & .089 & .220 & .672 \\
Authoritative Leadership1 & .421 & .289 & .653 \\
Authoritative Leadership2 & .297 & .142 & .545 \\
\hline
\end{tabular}

Extraction Method: Principal Component Analysis.

Rotation Method: Varimax with Kaiser Normalization

a. Rotation converged in 6 iterations.

Table 7-b. Rotated Component Matrix ${ }^{\mathrm{a}}$ of Authoritarian Leadership

\begin{tabular}{|l|r|r|}
\hline \multirow{2}{*}{} & \multicolumn{2}{|c|}{ Component } \\
\cline { 2 - 3 } & \multicolumn{1}{|c|}{1} & \multicolumn{1}{c|}{2} \\
\hline Authoritarian 1 & .869 & -.049 \\
Authoritarian 4 & .796 & .021 \\
Authoritarian 5 & .783 & -.189 \\
Authoritarian 2 & .749 & .324 \\
Authoritarian 3 & .690 & .281 \\
Authoritarian 6 & .011 & .931 \\
\hline
\end{tabular}

Extraction Method: Principal Component Analysis.

Rotation Method: Varimax with Kaiser Normalization.

${ }^{\text {a }}$. Rotation converged in 3 iterations. 
Table 8. Rotated Component Matrix ${ }^{\mathrm{a}}$ of Transformational Leadership

\begin{tabular}{|l|r|r|r|r|r|r|}
\hline & \multicolumn{5}{|c|}{ Component } \\
\cline { 2 - 6 } & \multicolumn{1}{|c|}{1} & \multicolumn{1}{c|}{2} & \multicolumn{1}{c|}{3} & \multicolumn{1}{c|}{4} & \multicolumn{1}{c|}{5} & \multicolumn{1}{c|}{6} \\
\hline Identify and Articulate a Vision 4 & .820 & .079 & -.116 & .065 & -.067 & -.062 \\
Identify and Articulate a Vision 1 & .750 & .207 & -.310 & .018 & .075 & -.215 \\
Identify and Articulate a Vision 3 & .733 & -.049 & .029 & -.064 & -.210 & .309 \\
Identify and Articulate a Vision 2 & .726 & .060 & .202 & .038 & .213 & .137 \\
Identify and Articulate a Vision 5 & .591 & -.097 & .051 & .242 & .173 & .404 \\
Provide an Appropriate Model 1 & .238 & .813 & .039 & .138 & .163 & .254 \\
Provide an Appropriate Model 2 & .033 & .767 & .146 & .127 & .057 & .210 \\
Provide an Appropriate Model 3 & -.041 & .720 & .103 & .276 & .177 & .202 \\
Provide Individualized Support 1 & .062 & -.011 & .881 & .084 & .044 & -.031 \\
Provide Individualized Support 4 & -.135 & -.010 & .838 & .301 & .134 & .082 \\
Provide Individualized Support 3 & .021 & .293 & .718 & -.091 & .374 & .087 \\
Provide Individualized Support 2 & -.073 & .289 & .685 & .241 & .024 & -.147 \\
Fostering the Acceptance of Group Goals 4 & .021 & .146 & .189 & .765 & .161 & .306 \\
Fostering the Acceptance of Group Goals 1 & .158 & .234 & .097 & .720 & .186 & .117 \\
Fostering the Acceptance of Group Goals 3 & -.115 & .559 & .178 & .577 & .014 & -.083 \\
Fostering the Acceptance of Group Goals 2 & .210 & .430 & .213 & .526 & .100 & .029 \\
Intellectual Stimulation 1 & -.054 & .044 & .083 & -.022 & .828 & .219 \\
Intellectual Stimulation 3 & .247 & .227 & .164 & .129 & .698 & .000 \\
Intellectual Stimulation 4 & -.124 & .005 & .076 & .399 & .649 & .070 \\
Intellectual Stimulation 2 & .046 & .148 & .137 & .486 & .586 & -.102 \\
High Performance Expectation 2 & .005 & .340 & -.017 & .187 & .011 & .754 \\
High Performance Expectation 3 & .161 & .267 & .081 & -.044 & .434 & .674 \\
High Performance Expectation 1 & .293 & .356 & -.228 & .245 & .020 & .522 \\
\hline
\end{tabular}

Extraction Method: Principal Component Analysis.

Rotation Method: Varimax with Kaiser Normalization.

a. Rotation converged in 9 iterations. 
Table 9. Rotated Component Matrix ${ }^{a}$ of Harmony

\begin{tabular}{|l|r|}
\hline \multirow{2}{*}{} & \multicolumn{1}{|c|}{ Component } \\
\cline { 2 - 2 } & \multicolumn{1}{|c|}{1} \\
\hline Harmony 1 & .618 \\
Harmony 2 & .691 \\
Harmony 3 & .740 \\
Harmony 4 & .851 \\
Harmony 5 & .695 \\
\hline
\end{tabular}

Extraction Method: Principal Component Analysis.

a. 1 components extracted.

Table 10. Rotated Component Matrix ${ }^{\mathrm{a}}$ of Trust

\begin{tabular}{|l|r|r|}
\hline \multirow{2}{*}{} & \multicolumn{2}{|c|}{ Component } \\
\cline { 2 - 3 } & \multicolumn{1}{|c|}{1} & \multicolumn{1}{c|}{2} \\
\hline Trust 1 & .647 & .135 \\
Trust 2 & .744 & .030 \\
Trust 3 & .771 & .265 \\
Trust 4 & .809 & .284 \\
Trust 5 & .150 & .915 \\
Trust 6 & .787 & -.401 \\
\hline
\end{tabular}

Extraction Method: Principal Component Analysis.

Rotation Method: Varimax with Kaiser Normalization.

a. Rotation converged in 3 iterations.

Table 11. Rotated Component Matrix ${ }^{a}$ of Effectiveness

\begin{tabular}{|l|r|r|}
\hline \multirow{2}{*}{} & \multicolumn{2}{|c|}{ Component } \\
\cline { 2 - 3 } & \multicolumn{1}{|c|}{1} & \multicolumn{1}{c|}{2} \\
\hline Effectiveness 1 & .627 & .280 \\
Effectiveness 2 & .014 & .936 \\
Effectiveness 3 & .600 & .533 \\
Effectiveness 4 & .865 & -.081 \\
Effectiveness 5 & .787 & .096 \\
\hline
\end{tabular}

Extraction Method: Principal Component Analysis.

Rotation Method: Varimax with Kaiser Normalization.

a. Rotation converged in 3 iterations. 
Coefficient alpha, or Cronbach's alpha (Nunnally, 1978) was used for all scales to assess internal consistency of all scales. All Cronbach's alpha indices exceeded .70 suggesting that the questions are internally consistent. The Cronbach's alpha index for the "providing an appropriate model" dimension of transformational leadership could be improved from .85 to .86 if "Provide an Appropriate Model 3" is deleted. The Cronbach's alpha index for the paternalistic leadership scale could be improved from .85 to .86 if "Authoritative leadership 4" is deleted. Other than items on the authoritarian leadership, trust, and effectiveness, scales that loaded on a second factor, which were deleted, all other items on other scales were retained because improvement in Cronbach's alpha was negligible when items were removed. All Cronbach's alphas indices are presented in Table 12. All measurement scales, reflecting revisions, are presented in Appendix II.

Table 12. Results of Cronbach's alpha

\begin{tabular}{|l|l|}
\hline Scales & Cronbach's alpha Index \\
\hline Benevolent Leadership & .85 \\
Moral Leadership & .87 \\
Authoritative Leadership & .78 \\
Authoritarian Leadership & .84 \\
Overall Paternalistic Leadership & .85 \\
Identify and Articulate a Vision & .79 \\
Provide an Appropriate Model & .85 \\
Fostering the Acceptance of Group Goals & .80 \\
High Performance Expectation & .73 \\
Provide Individualized Support & .85 \\
Intellectual Stimulation & .76 \\
Overall Transformational leadership & .84 \\
Trust & .80 \\
Harmony & .75 \\
Effectiveness & .74 \\
\hline
\end{tabular}

Next, an analysis of bivariate correlations between dimensions of paternalistic leadership was conducted. In this study, I used the dimension of authoritative leadership to replace 
authoritarian leadership in the paternalistic leadership measure so that all dimensions of paternalistic leadership would positively correlate with each other, and would similarly correlate with other study variables. The results in Table 13 reveal that different from authoritarian leadership, which negatively correlates with other dimensions of paternalistic leadership, authoritative leadership significantly positively correlates with both moral and benevolent leadership.

Table 13. Correlations Matrix of Paternalistic Dimensions

\begin{tabular}{|l|c|c|c|c|}
\hline & 1 & 2 & 3 & 4 \\
\hline 1. Moral Leadership & 1.00 & & & \\
\hline 2. Benevolent Leadership & $.601^{* *}$ & 1.00 & & \\
\hline 3. Authoritative Leadership & $.334^{* *}$ & $.513^{* *}$ & 1.00 & \\
\hline 4. Authoritarian Leadership & $-.419^{* *}$ & $-.255^{*}$ & .093 & 1.00 \\
\hline
\end{tabular}

**. Correlation is significant at the 0.01 level.

*. Correlation is significant at the 0.05 level.

An analysis of bivariate correlations between all study variables also was conducted. According to the results, both paternalistic leadership (using authoritative leadership) and transformational leadership positively correlate with trust, harmony, and effectiveness. Both harmony and trust are positively correlated with effectiveness. The intercorrelations of these variables are reported in Table 14.

Table 14. Correlations Matrix of Predictors and Outcomes

\begin{tabular}{|l|c|c|c|c|c|}
\hline & 1 & 2 & 3 & 4 & 5 \\
\hline 1. Paternalistic Leadership & $.85^{a}$ & & & & \\
\hline 2. Transformational Leadership & $.762^{* *}$ & $.84^{a}$ & & & \\
\hline 3. Trust & $.473^{* *}$ & $.453^{*}$ & $.80^{a}$ & & \\
\hline 4. Harmony & $.593^{* *}$ & $.639^{* *}$ & $.752^{* *}$ & $.75^{a}$ & \\
\hline 5. Effectiveness & $.555^{* *}$ & $.580^{* *}$ & $.707^{* *}$ & $.744^{* *}$ & $.74^{a}$ \\
\hline
\end{tabular}

**. Correlation is significant at the 0.01 level.

* Correlation is significant at the 0.05 level.

${ }^{a}$. Cronbach's alpha is italicized on the diagonal.

Next, regression and mediation analyses were conducted to determine whether the relationships between the variables were as expected. The effects of paternalistic leadership and 
transformational leadership on effectiveness are proposed to be partially mediated by trust and harmony. I used Baron and Kenny's (1986) four-step process to test the mediational hypotheses because it is the most common method for testing mediation in the social sciences (MacKinnon, Lockwood, Hoffman, West, \& Sheets, 2002). According to this method, the first step is to show both paternalistic leadership and transformational leadership are correlated with leadership effectiveness (see Path c in Figure 6). Step 2 is to show both types of leadership are correlated with the mediators, trust and harmony (Path a in Figure 6). The third step is to show both mediators are correlated with leadership effectiveness (see Path b in Figure 6). The final step is to show the mediator effects of trust and harmony on the leadership style-effectiveness relationship, by finding that the strength of the relationships between the leadership behaviors and effectiveness are reduced when the mediators (e.g., trust and harmony) are added to the model (compare Path c with Path c' in Figure 4).

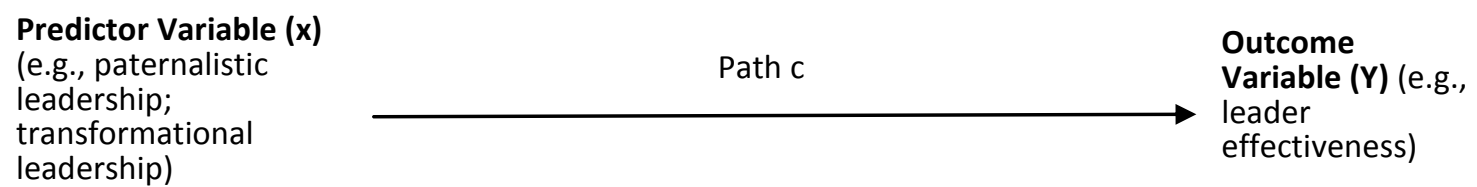

Path $c^{\prime}$

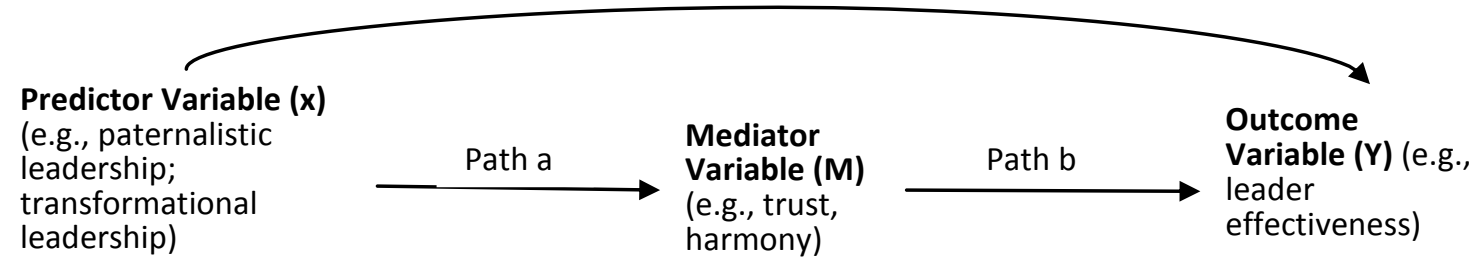

Figure 4. Diagram of paths in mediation models

Table 15 contains the results from the mediational hypotheses tests. For hypothesis 1, it states that subordinates' trust in leader partially mediates the relationship between paternalistic 
leadership and leader effectiveness. The results indicate that paternalistic leadership was related to effectiveness (Path $\mathrm{c})$ as the unstandardized regression coefficient $(B=.16)$ associated with the effect of paternalistic leadership on effectiveness was significant $(p<.0001)$. The unstandardized regression coefficient $(B=.19)$ associated with the relationship between paternalistic leadership and trust (Path a) also was significant $(p<.0001)$. To test whether trust was related to effectiveness (Path b), I regressed effectiveness simultaneously on both trust and the paternalistic leadership variable. The coefficient associated with the relation between trust and effectiveness (controlling for paternalistic leadership; Path b) was significant $(B=.42, p<$ .0001). The results show that the relationship between paternalistic leadership and effectiveness (Path c') was .08 and still significant $(p<.05)$, which means trust partially mediates the relation between paternalistic leadership and effectiveness. To test whether the drop (from $B=.16$ in Path c to $B=.08$ in Path c') is significant, I used the Sobel test. The Sobel test determines the significance of the indirect effect of the mediator by testing the hypothesis of no difference between the direct effect (path c) and the indirect effect (path c'). Performing the Sobel test can directly test for a reduction in the effect of the independent variable on the dependent variable when controlling for the mediator. The result indicates that the partial mediation is significant (Sobel test score $=3.82, p<.0001)$.

Hypothesis 2 states that subordinates' trust in leader partially mediates the relationship between transformational leadership and leader effectiveness. The results indicate that transformational leadership was related to effectiveness (Path $\mathrm{c}$ ) as the unstandardized regression coefficient $(B=.16)$ associated with the effect of transformational leadership on effectiveness was significant $(p<.0001)$. The unstandardized regression coefficient $(B=.18)$ associated with the relationship between transformational leadership and trust (Path a) also was significant ( $p<$ 
.0001). To test whether trust was related to effectiveness (Path b), I regressed effectiveness simultaneously on both trust and the transformational leadership variable. The coefficient associated with the relation between trust and effectiveness (controlling for transformational leadership; Path $\mathrm{b})$ was significant $(B=.39, p<.0001)$. The results indicate that the relationship between transformational leadership and effectiveness (Path c') was .09 and still significant ( $p<$ .0001), which means trust partially mediates the relation between transformational leadership and effectiveness. To test whether the $\operatorname{drop}$ (from $B=.16$ in Path $\mathrm{c}$ to $B=.09$ in Path c') is significant, I used the Sobel test. The result indicates that the partial mediation is significant (Sobel test score $=3.85, p<.0001$ ).

Hypothesis 3 states that the degree of harmony in the organization partially mediates the relationship between paternalistic leadership and its effectiveness on subordinates. The results indicate that paternalistic leadership was related to effectiveness (Path c) as the unstandardized regression coefficient $(B=.16)$ associated with the effect of paternalistic leadership on effectiveness was significant $(p<.0001)$. The unstandardized regression coefficient $(B=.21)$ associated with the relationship between paternalistic leadership and harmony (Path a) also was significant $(p<.0001)$. To test whether trust was related to effectiveness (Path b), I regressed effectiveness simultaneously on both harmony and the paternalistic leadership variable. The coefficient associated with the relation between trust and effectiveness (controlling for paternalistic leadership; Path $\mathrm{b})$ was significant $(B=.53, p<.0001)$. The results indicate that the relationship between paternalistic leadership and effectiveness (Path c') was .05, but not significant, which means harmony partially mediates the relation between paternalistic leadership and effectiveness. To test whether the drop (from $B=.16$ in Path $\mathrm{c}$ to $B=.05$ in Path 
c') is significant, I used the Sobel test. The result indicates that the partial mediation is significant $($ Sobel test score $=5.01, p<.0001)$.

Hypothesis 4 states that the degree of harmony in the organization partially mediates the relationship between transformational leadership and its effectiveness on subordinates. The results indicate that transformational leadership was related to effectiveness (Path c) as the unstandardized regression coefficient $(B=.16)$ associated with the effect of transformational leadership on effectiveness was significant $(p<.0001)$. The unstandardized regression coefficient $(B=.22)$ associated with the relationship between transformational leadership and harmony (Path a) also was significant $(p<.0001)$. To test whether trust was related to effectiveness (Path b), I regressed effectiveness simultaneously on both harmony and the transformational leadership variable. The coefficient associated with the relation between trust and effectiveness (controlling for transformational leadership; Path $\mathrm{b}$ ) was significant $(B=.50, p$ $<.0001)$. The results indicate that the relationship between transformational leadership and effectiveness (Path c') was .06, but not significant, which means harmony partially mediates the relation between transformational leadership and effectiveness. To test whether the drop (from $B$ $=.16$ in Path c to $B=.06$ in Path c') is significant, I used the Sobel test. The result indicates that the partial mediation is significant (Sobel test score $=5.38, p<.0001$ ). Therefore, hypotheses 1 through 4 are supported. 
Table 15. Testing Mediator Effects

H1: Mediating effects of TRUST on relationship between paternalistic leadership and effectiveness

\begin{tabular}{|c|c|c|c|c|}
\hline$\overline{\text { Steps }}$ & $\mathrm{B}$ & SE B & $95 \% \mathrm{CI}$ & $\beta$ \\
\hline \multirow{2}{*}{\multicolumn{5}{|c|}{$\begin{array}{l}\text { Path c } \\
\text { Outcome: Effectiveness }\end{array}$}} \\
\hline & & & & \\
\hline $\begin{array}{l}\text { Predictor: Paternalistic Leadership } \\
\text { Path a }\end{array}$ & .16 & .03 & $.10, .22$ & $.551 * *$ \\
\hline Outcome: Trust & & & & \\
\hline $\begin{array}{l}\text { Predictor: Paternalistic Leadership } \\
\text { Path b and c' }\end{array}$ & .19 & .04 & $.14, .28$ & $.48^{* *}$ \\
\hline Outcome: Effectiveness & & & & \\
\hline Mediator: Trust (Path b) & .42 & .07 & $.28, .56$ & $.57 * *$ \\
\hline Predictor: Paternalistic Leadership (Path c') & .08 & .03 & $.24, .13$ & $.28 *$ \\
\hline
\end{tabular}

H2: Mediating effects of TRUST on relationship between transformational leadership and effectiveness

\begin{tabular}{|c|c|c|c|c|}
\hline Steps & B & SE B & $95 \% \mathrm{CI}$ & $\beta$ \\
\hline $\begin{array}{l}\text { Path c } \\
\text { Outcome: Effectiveness }\end{array}$ & & & & \\
\hline $\begin{array}{l}\text { Predictor: Transformational Leadership } \\
\text { Path a } \\
\text { Outcome: Trust }\end{array}$ & .16 & .03 & $.11, .22$ & $.60 * *$ \\
\hline $\begin{array}{l}\text { Predictor: Transformational Leadership } \\
\text { Path b and c' } \\
\text { Outcome: Effectiveness }\end{array}$ & .18 & .04 & $.10, .26$ & $.48^{* *}$ \\
\hline Mediator: Trust (Path b) & .39 & .07 & $.26, .53$ & $.54 * *$ \\
\hline $\begin{array}{l}\text { Predictor: Transformational Leadership } \\
\text { (Path c') }\end{array}$ & .09 & .03 & $.04, .14$ & $.34 * *$ \\
\hline
\end{tabular}

H3: Mediating effects of HARMONY on relationship between paternalistic leadership and effectiveness

\begin{tabular}{|c|c|c|c|c|}
\hline Steps & $\mathrm{B}$ & SE B & $95 \% \mathrm{CI}$ & $\beta$ \\
\hline $\begin{array}{l}\text { Path c } \\
\text { Outcome: Effectiveness }\end{array}$ & & & & \\
\hline $\begin{array}{l}\text { Predictor: Paternalistic Leadership } \\
\text { Path a } \\
\text { Outcome: Harmony }\end{array}$ & .16 & .03 & $.10, .22$ & $.55^{* *}$ \\
\hline $\begin{array}{l}\text { Predictor: Paternalistic Leadership } \\
\text { Path b and c' } \\
\text { Outcome: Effectiveness }\end{array}$ & .21 & .04 & $.14, .28$ & $.60 * *$ \\
\hline Mediator: Harmony (Path b) & .53 & .08 & $.36, .69$ & $.65^{* *}$ \\
\hline Predictor: Paternalistic Leadership (Path c') & .05 & .03 & $-.01, .13$ & .16 \\
\hline
\end{tabular}


H4: Mediating effects of HARMONY on relationship between transformational leadership and effectiveness

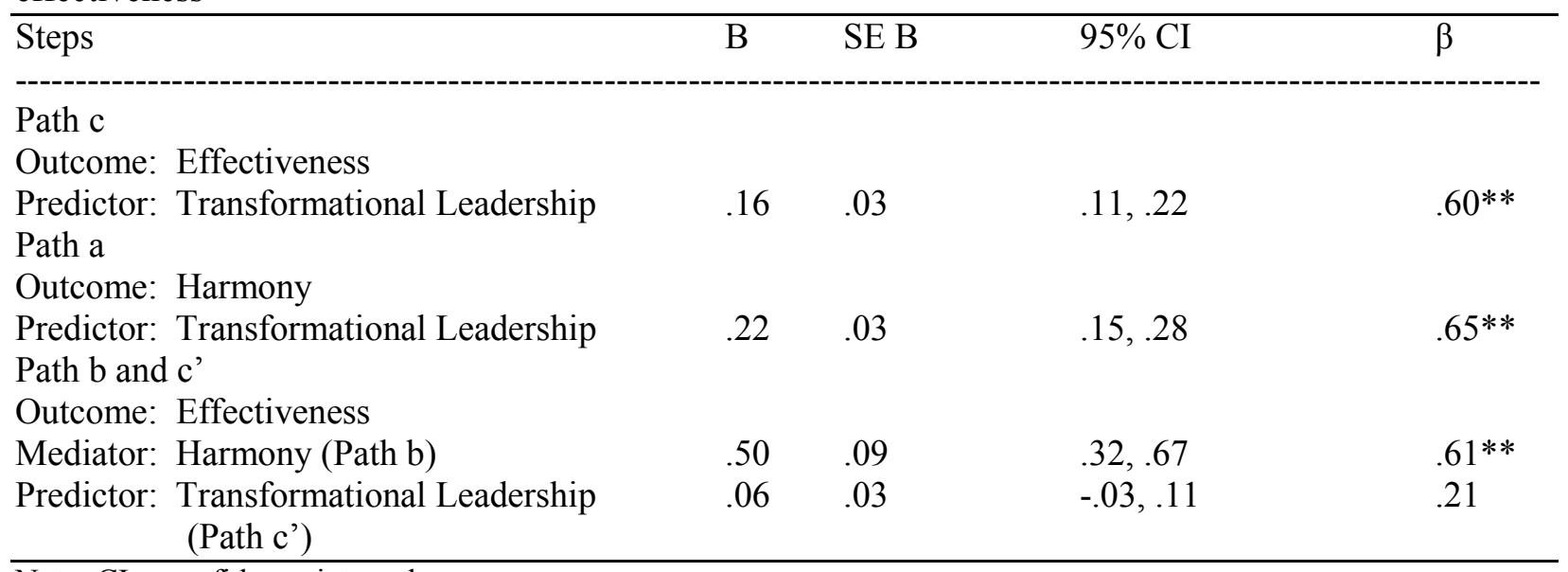

Note: $\mathrm{CI}=$ confidence interval;

$* * p<.05, * p<.10$.

Hierarchical regression was used to test moderator hypotheses 5 through 8 . Statisticians generally encourage the use of hierarchical regression techniques over the practice of comparing correlations between groups when the group variable is naturally categorical (Baron \& Kenny, 1986). In this study, generation is the categorical moderator, paternalistic and transformational leadership are independent variables, and trust and harmony are dependent variables. First, I used dummy coding for the categorical variable, generation. Dummy coding (Generation X was coded as 1 and Generation Y was coded as 0 ) is used when comparisons with a control or base group are desired (Frazier, Tix, \& Barron, 2004). The next step involved centering the independent variables (e.g., paternalistic leadership, transformational leadership). Centering reduces problems associated with multicollinearity among the variables in the regression (Cohen, Cohen, West, \& Aiken, 2003). Then, product terms were created that represent the interaction between the predictor (e.g., paternalistic leadership, transformational leadership) and the moderator (e.g., generation). To form product terms, I multiplied the centered predictor with the newly coded moderator variables. Finally, a hierarchical regression involving two steps was run to test moderating hypotheses. In the first step, the main effect variables were entered into the 
regression. This included the independent variables (e.g., paternalistic leadership,

transformational leadership) and moderator variable (generation). The second step was to add

interaction terms in Block 2 of 2 . The change in $\mathrm{R}^{2}$ between steps 1 and 2 was examined to

determine whether the moderator was significant. Results are reported in Table 16.

Table 16. Testing Moderator Effects

H5: Moderator effects of Generation on relationship between paternalistic leadership and trust

\begin{tabular}{|c|c|c|c|c|}
\hline Steps and variable & B & $\beta$ & $\mathrm{R}^{2}$ & $\Delta \mathrm{R}^{2}$ \\
\hline \multicolumn{5}{|c|}{ Dummy coding (Generation X coded 1 , Generation $Y$ coded 0 ) } \\
\hline Model 1 & & & $.28 * *$ & $.28^{* *}$ \\
\hline Paternalistic Leadership & $.16^{* *}$ & .40 & & \\
\hline Generation & $-1.67 *$ & -.23 & & \\
\hline Model 2 & & & $.28 * *$ & .00 \\
\hline Paternalistic Leadership & .10 & .24 & & \\
\hline Generation & $-1.79 *$ & -.25 & & \\
\hline Paternalistic Leadership $\times$ Generation & .08 & .17 & & \\
\hline \multicolumn{5}{|c|}{ H6: Moderator effects of Generation on relationship between transformational leadership and trust } \\
\hline Steps and variable & $\mathrm{B}$ & $\beta$ & $\mathrm{R}^{2}$ & $\Delta \mathrm{R}^{2}$ \\
\hline \multicolumn{5}{|c|}{ Dummy coding (Generation X coded 1 , Generation $Y$ coded 0 ) } \\
\hline Model 1 & & & $.28 * *$ & $.28 * *$ \\
\hline Transformational Leadership & $.15^{* *}$ & .40 & & \\
\hline Generation & $-1.72 *$ & -.24 & & \\
\hline Model 2 & & & $.28 * *$ & .00 \\
\hline Transformational Leadership & $.16^{*}$ & .42 & & \\
\hline Generation & $-1.71 *$ & -.24 & & \\
\hline Transformational Leadership $\times$ Generation & -.01 & -.02 & & \\
\hline \multicolumn{5}{|c|}{ H7: Moderator effects of Generation on relationship between paternalistic leadership and harmony } \\
\hline Steps and variable & B & $\beta$ & $\mathrm{R}^{2}$ & $\Delta \mathrm{R}^{2}$ \\
\hline \multicolumn{5}{|c|}{ Dummy coding (Generation X coded 1 , Generation Y coded 0 ) } \\
\hline Model 1 & & & $.39 * *$ & $.39 * *$ \\
\hline Paternalistic Leadership & $.19 * *$ & .53 & & \\
\hline Generation & -1.26 & -.20 & & \\
\hline Model 2 & & & $.42 * *$ & .03 \\
\hline Paternalistic Leadership & .07 & .20 & & \\
\hline Generation & $-1.49 *$ & -.23 & & \\
\hline Paternalistic Leadership $\times$ Generation & .15 & .36 & & \\
\hline
\end{tabular}


H8: Moderator effects of Generation on relationship between transformational leadership and harmony

\begin{tabular}{|c|c|c|c|c|}
\hline Steps and variable & B & $\beta$ & $\overline{\mathrm{R}^{2}}$ & $\Delta \mathrm{R}^{2}$ \\
\hline \multicolumn{5}{|c|}{ Dummy coding (Generation X coded 1 , Generation $Y$ coded 0 ) } \\
\hline Model 1 & & & $.45^{* *}$ & $.45^{* *}$ \\
\hline Transformational Leadership & $.20 * *$ & .59 & & \\
\hline Generation & -1.20 & -.19 & & \\
\hline Model 2 & & & $.46^{* *}$ & .01 \\
\hline Transformational Leadership & $.15^{*}$ & .42 & & \\
\hline Generation & -1.25 & -.24 & & \\
\hline Transformational Leadership $\times$ Generation & -.07 & .17 & & \\
\hline
\end{tabular}

Note: $\mathrm{CI}=$ confidence interval;

$* * p<.05, * p<.10$

Hypothesis 5 states that generation moderates the relationship between paternalistic leadership and subordinates' trust in leader, such that the positive relationship between paternalistic leadership and trust is stronger for Generation X than for Generation Y. In model 1, results indicate that paternalistic leadership and generation significantly predicted trust $(\beta=.40$, $p<.001 ; \beta=-.23, p<.001$, respectively). The change in $\mathrm{R}^{2}$ after adding the interaction variable in model 2 was not significant $\left(\Delta \mathrm{R}^{2}=.00\right)$; therefore, hypothesis 5 is not supported.

Hypothesis 6 states that generation moderates the relationship between transformational leadership and subordinates' trust in leader, such that the positive relationship between transformational leadership and trust is stronger for Generation $\mathrm{Y}$ than for Generation X. In model 1 , results indicate that transformational leadership and generation significantly predicted trust $\left(\beta=.40, p<.001 ; \beta=-.24, p<.001\right.$, respectively). The change in $\mathrm{R}^{2}$ after adding the interaction variable in model 2 was not significant $\left(\Delta \mathrm{R}^{2}=.00\right)$, therefore, hypothesis 6 is not supported.

Hypothesis 7 states that generation moderates the relationship between paternalistic leadership and harmony between a leader and his/her subordinates, such that the positive relationship between paternalistic leadership and harmony is stronger for Generation $\mathrm{X}$ than for Generation Y. In model 1, results show that paternalistic leadership significantly predicted 
harmony $(\beta=.53, p<.001)$. The change in $\mathrm{R}^{2}$ after adding the interaction variable in model 2 was not significant $\left(\Delta R^{2}=.03\right)$; therefore, hypothesis 7 is not supported.

Hypothesis 8 states that generation moderates the relationship between transformational leadership and harmony between a leader and his/her subordinates, such that the positive relationship between transformational leadership and harmony is stronger for Generation $\mathrm{Y}$ than for Generation X. In model 1, results show that transformational leadership significantly predicted harmony $(\beta=.59, p<.001)$. The change in $\mathrm{R}^{2}$ after adding the interaction variable in model 2 was not significant $\left(\Delta R^{2}=.01\right)$, therefore, hypothesis 8 is not supported. Results indicate hypotheses 5 through 8 are not supported. It is important to note, however, that the results may be because of an insufficient sample size; larger sample sizes yield smaller p-values (Frazier et al., 2004). Another possible reason may be due to unequal numbers of participants for different levels of a categorical variable (37 for Generation $\mathrm{Y}$ and 29 for Generation X). If sample sizes are unequal across groups, power can be reduced (Frazier et al., 2004).

Although the regression analyses do not support any moderating effects, graphs of the slopes provide an indication as to whether the direction of the proposed moderator effect is as predicted. Employees from Generation X do tend to improve their perceptions of trust and harmony at a greater rate as paternalistic leadership increases than those from Generation Y. Employees from Generation $\mathrm{Y}$ appear to improve their perceptions of harmony at a greater rate as transformational leadership increases than those from Generation X; however, the reverse is true for trust (see Figures 5,6,7, and 8). 
Moderator Effect of Generation on the Paternalistic Leadership-Trust Relationship

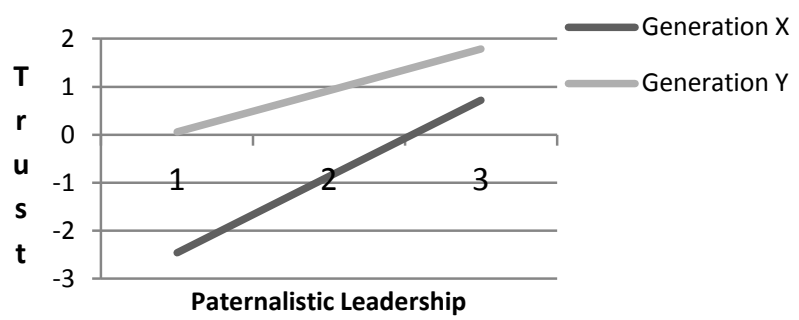

Figure 5. Moderator Effect of Generation on the Paternalistic Leadership-Trust Relationship

Moderator Effect of Generation on the Transformational Leadership -Trust Relationship
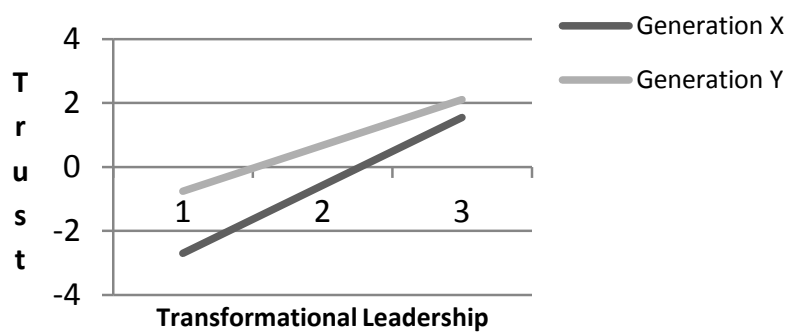

Figure 7. Moderator Effect of Generation on the Transformational Leadership-Trust Relationship
Moderator Effect of Generation on the Paternalistic Leadership-Harmony Relationship

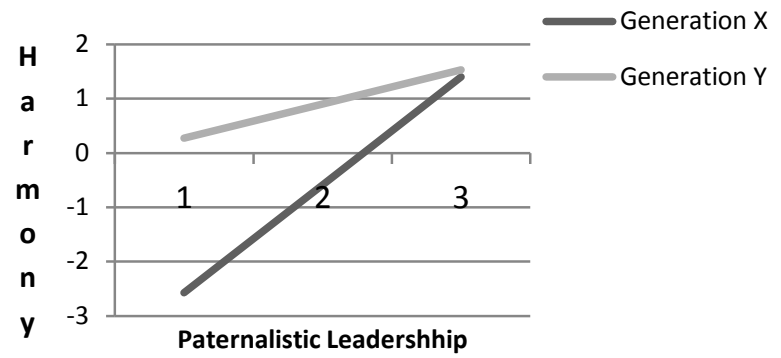

Figure 6. Moderator Effect of Generation on the Paternalistic Leadership-Harmony Relationship

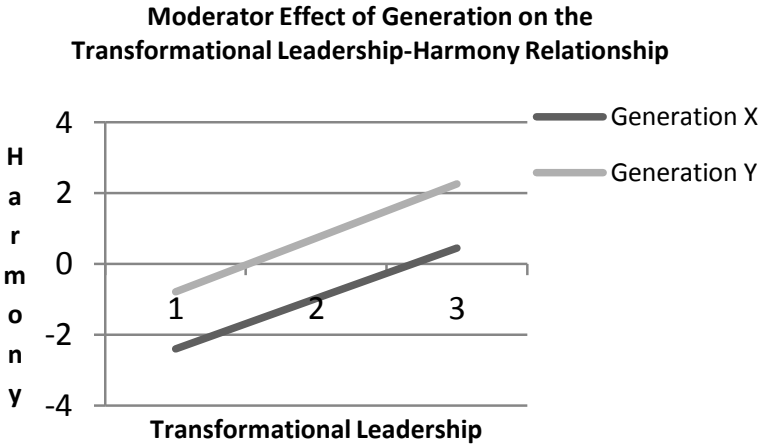

Figure 8. Moderator Effect of Generation on the Transformation LeadershipHarmony Relationship

Summary and Revisions to the Main Study

By conducting a pilot study, I was able to examine the characteristics of the data and the instruments. It helped to confirm the validity and reliability of the instruments and provide a pre-analysis of the hypotheses. The pilot study also helped to improve the clarity and wording of the survey based on the suggestions received from the respondents and the results of the data analyses.

All the items in the survey were clear and understood by the respondents. The pilot study reveals that some specifications of the questions are helpful. For example, question number 52 asks to what degree the supervisor holds employees accountable for work rules and high 
performance standards. A specification was added in the Chinese version that clarifies the high performance standards refer to employees rather than the supervisor. According to the factor loading results, item Trust 5, Effectiveness 2, and Authoritarian leadership 6 were deleted so that the trust, effectiveness, and authoritarian leadership scales loaded as single factors. Therefore, the final total questionnaire items are 63 after scale purification, which results the sample size for the full study is $315(63 \times 5)$. Although this number of sample size is more than the number 97 , which was calculated according to formula $\mathrm{n}=\left(\lambda / f^{2}\right)+(k+1)$ mentioned in earlier sample section, it will allow more space for matching the number across two groups in Generation $\mathrm{X}$ and $\mathrm{Y}$ to gain more power.

The feedback from the respondents also suggested that some changes needed to be made in how questions were asked for the demographic variables. Question number 6 asks how long the employees have been in their company. When asked, most of the respondents admitted they answered how many years they worked for the department or branch rather than the company. Therefore, a clarification that this question refers to how long the respondents have been in the company rather than in the department or branch will be added after the question. In addition, a question that asks how long employees have worked for their supervisors will be added because the duration of that relationship may relate to trust and harmony. Finally, the response set to question number 7 that asks for educational information has been restructured. Chinese do not use the term associate's degree; instead, they refer to two-year college degrees. Therefore, Response number 3, associate's degree, will be replaced with "two-year college degree." This chapter concerns the methods used to test the hypotheses, which were presented in Chapter II. The data collection plan, sampling procedure, power, effect size, sample size and data analysis techniques were discussed in this chapter. Further, a pilot study was conducted to 
ensure the quality and appropriateness of the newly developed instrument. Results of the pilot study were discussed and changes have been made based on the results and suggestions from respondents. The next step will be to defend this proposal and more into the data collection stage for the full study. 


\section{CHAPTER IV}

\section{DATA ANALYSIS AND RESULTS}

This chapter provides results of statistical analyses used to test hypotheses presented in chapter two. In doing so, the survey response rate and characteristics of the sample are presented first. Next, results of the factor analysis of the newly constructed variables, internal consistency reliabilities and validity checks are presented. Following the discussion on validity and reliability of variables, descriptive statistics and regression analysis results are presented.

\section{Survey Response Rate and Sample Characteristics}

An on-site survey method was used to collect data from nine organizations in China. The survey consisted of 76 questions. The first page of the survey included an introduction to the survey, the time to complete, contact information, and instructions. The body of the survey includes two sections. The first section is comprised of demographic information questions such as gender, age, education, and organizational tenure, etc. In the second section, respondents were asked to give opinions about leadership characteristics of their current managers, degree of trust they have in their leaders, degree of harmonious relationships between managers and themselves, and their perceptions of leadership effectiveness. The surveys were conducted on site at different times across different enterprises. Written instructions were attached as a cover page of the survey and detailed instructions were explained verbally at the beginning of the survey administration. The surveys were collected immediately by an administrator outside the company upon completion.

The design of this study presented minimal risk to the participants, as it involved no experiential treatments of the subjects or exposure to physical or psychological harm. There was no formal debriefing of the participants after the study, but participant companies will be 
supplied with an executive summary of the findings if requested. Individual participants may also request a summary. No sanctions or incentives were used to encourage participation, nor were any applied if the participants declined or withdrew from the study. No information regarding participation of any individuals was communicated to their respective organizations. Confidentiality of data was maintained at all times and identification of individual named responses was not available. These conditions were communicated to all participants at the beginning of the survey. The sample size consisted of 312 full-time employees in China. Onsite survey collection provided a $100 \%$ response rate. As response rate is an important indicator of survey quality, the higher response rates assure more accurate survey results (Aday, 1996). The 312 useable responses represent a wide variety of Chinese organizations across different industries. Table 17 includes the characteristics of the samples regarding their basic work in their company. Two hundred and seven respondents $(66 \%)$ were from private companies while 105 respondents (34\%) were from state-owned companies. In Table 18 the size of the companies is presented. In terms of company geographical location, 211 responses were from Changsha, 88 were from Xiangxiang, and 13 were from Hong Kong.

Table 17. Characteristics of the Basic Work of the Company

\begin{tabular}{llcc}
\hline & Frequency & Percent & Cumulative Percent \\
\hline Retail & 12 & 3.8 & 3.8 \\
Wholesale & 59 & 18.9 & 22.8 \\
Manufacturing & 185 & 59.3 & 82.1 \\
Service & 39 & 12.5 & 94.6 \\
Trading & 17 & 5.4 & 100 \\
\hline Total & 312 & 100 & \\
\hline
\end{tabular}


Table 18. Size of the Company

\begin{tabular}{llcc}
\hline Size (\# of employees) & Frequency (\# of respondents) & Percent & Cumulative Percent \\
\hline 16 & 13 & 4.2 & 4.2 \\
$40-45$ & 39 & 12.5 & 16.7 \\
$70-75$ & 17 & 5.4 & 22.1 \\
$80-85$ & 24 & 7.7 & 29.8 \\
113 & 97 & 31.1 & 60.9 \\
$440-450$ & 12 & 3.8 & 64.7 \\
$800-850$ & 22 & 7.1 & 71.8 \\
$2300-2500$ & 38 & 12.2 & 84 \\
$3000-3100$ & 50 & 16 & 100 \\
\hline Total & 312 & 100 & \\
\hline
\end{tabular}

Demographic data was collected to better understand respondents' demographic background and compare the general demographic characteristics of the Chinese employees. The sample was predominantly male and most of them were low level employees such as steel plant workers, sales people, and general clerks. Tables 19 and 20 present the characteristics of the samples regarding gender and positions. Table 21 contains information on respondents' tenure. The average length of service in an organization was 6.65 years. Table 22 presents data on the number of years that the respondents had worked with the current supervisor. The average length of time was 3.52 years.

Table 19. Gender

\begin{tabular}{llcc}
\hline Gender & Frequency & Percent & Cumulative Percent \\
\hline Male & 205 & 65.7 & 65.7 \\
Female & 107 & 34.3 & 100 \\
\hline Total & 312 & 100 & \\
\hline
\end{tabular}


Table 20. Respondents' Position

\begin{tabular}{llll}
\hline & Frequency & Percent & Cumulative Percent \\
\hline Accountant & 28 & 8.9 & 8.9 \\
General Clerk & 34 & 10.9 & 19.8 \\
Engineer & 10 & 3.2 & 23 \\
Manager & 5 & 1.6 & 24.6 \\
Musician & 17 & 5.4 & 30 \\
Sales & 55 & 17.6 & 47.6 \\
Technician & 24 & 7.7 & 55.3 \\
Imp. \& Exp. Trader & 14 & 4.5 & 59.7 \\
Steel Plant Worker & 126 & 40.3 & 100 \\
\hline Total & 312 & 100 &
\end{tabular}

Table 21. Organizational Tenure

\begin{tabular}{llll}
\hline & Frequency & Percent & Cumulative Percent \\
\hline 0-2 years & 80 & 25.6 & 25.6 \\
3-5 years & 155 & 36.9 & 62.5 \\
6-10 years & 58 & 18.6 & 81.1 \\
More than 10 years & 59 & 18.9 & 100 \\
\hline Total & 312 & 100 & \\
\hline
\end{tabular}

Table 22. Years Working with the Current Supervisor

\begin{tabular}{llcc}
\hline & Frequency & Percent & Cumulative Percent \\
\hline 0-2 years & 160 & 51.3 & 51.3 \\
3-5 years & 119 & 38.2 & 89.4 \\
6-10 years & 20 & 6.5 & 95.8 \\
More than 10 years & 16 & 4.1 & 100 \\
\hline Total & 312 & 100 & \\
\hline
\end{tabular}

A principal focus of the study is generation identity. Respondents aged 30 or under are categorized as Generation Y while those aged 31 or older are categorized as Generation X. Table 23 presents the characteristics of the samples regarding age. As shown in Table 23, there were more respondents $(56.1 \%)$ in the Generation Y group. With regard to education level, $36.2 \%$ of the respondents held a high school diploma, $19.8 \%$ held a vocational/technical 
certificate, $23.1 \%$ held a two-year college degree, $20.2 \%$ held a Bachelor's degree, and $1 \%$ held a Master's degree. Table 24 presents the educational level of the respondents.

Table 23. Age of the Respondents

\begin{tabular}{llll}
\hline & Frequency & Percent & Cumulative Percent \\
\hline Below 25 & 86 & 27.6 & 27.6 \\
$25-30$ & 89 & 28.5 & 56.1 \\
$31-35$ & 32 & 10.3 & 66.4 \\
$36-44$ & 41 & 13.1 & 79.5 \\
$45-54$ & 53 & 17 & 96.5 \\
$55-64$ & 11 & 3.5 & 100 \\
\hline Total & 312 & 100 & \\
\hline
\end{tabular}

Table 24. Education of the Respondents

\begin{tabular}{llcc}
\hline & Frequency & Percent & Cumulative Percent \\
\hline High school diploma & 113 & 36.2 & 36.2 \\
Vocational/technical certificate & 61 & 19.8 & 55.8 \\
Two-year college degree & 72 & 23.1 & 78.8 \\
Bachelor's degree & 63 & 20.2 & 99 \\
Master's degree & 3 & 1 & 100 \\
\hline Total & 312 & 100 & \\
\hline
\end{tabular}

\section{Measure Assessment}

In this section, the steps used to generate and evaluate the measurements and variables of the study are discussed. The issues related to internal consistency, validity, and reliability of the constructs and measures employed are addressed.

\section{Factor Analysis}

Factor analysis is a multivariate statistical technique that is used to analyze the structure of the correlations among a large number of variables based on a set of common underlying dimensions (Hair et al., 1998). An exploratory factor analysis was conducted using SPSS before hypothesis testing because of the newness of some of the survey questionnaires on questions for 
each construct. The criterion for item deletion was based on three rules: 1) an item-correlation that is lower than .40;2) high cross-loadings on two factors; and 3) subjective assessment of the importance of the item for capturing the domain represented by the construct (Churchill, 1979; Nunnally, 1978). Tables 25 to 29 include the results of the factor analyses on all the variables. The results show that items for paternalistic leadership loaded clearly into three separate dimensions as previous research indicates (Cheng et al., 2000). Items for transformational leadership loaded into six separate dimensions as suggested in previous research (Podsakoff et al., 1990). All five items of trust and all five items of harmony loaded as one component (see Tables 27 and 28). All four items of leadership effectiveness loaded as one component (see Table 29). Evidence from Tables 25 to 29 provided support for the measurement instruments used in the study, as the factor loadings were consistent with original scales.

Table 25. Factor Analysis of Paternalistic Leadership

\begin{tabular}{llll}
\hline Variable & Factor 1 & Factor 2 & Factor 3 \\
\cline { 2 - 4 } AVL1 & Authoritative Leadership & Moral Leadership & Benevolent Leadership \\
\cline { 2 - 3 } AVL2 & .555 & & \\
AVL3 & .498 & & \\
AVL4 & .772 & & \\
AVL5 & .667 & & \\
ML1 & .779 & .818 & \\
ML2 & & .798 & \\
ML3 & & .804 & \\
ML4 & & .751 & \\
ML5 & & .696 & .738 \\
BL1 & & .693 \\
BL2 & & .650 \\
BL3 & & & .710 \\
BL4 & & & .736 \\
BL5 & & & .669 \\
BL6 & & .20 & .83 \\
\hline Total Variance Explained & .21 & .85 & \\
\hline Crohbach's Alpha & .79 &
\end{tabular}


Table 26. Factor Analysis of Transformational Leadership

\begin{tabular}{|c|c|c|c|c|c|c|}
\hline Variable & Factor 1 & Factor 2 & Factor 3 & Factor 4 & Factor 5 & Factor 6 \\
\hline & $\begin{array}{l}\text { Identify a } \\
\text { Vision }\end{array}$ & $\begin{array}{l}\text { Provide a } \\
\text { Model }\end{array}$ & $\begin{array}{l}\text { Foster } \\
\text { Group }\end{array}$ & $\begin{array}{l}\text { High } \\
\text { Expectation }\end{array}$ & $\begin{array}{l}\text { Individualized } \\
\text { Support }\end{array}$ & $\begin{array}{l}\text { Intellectual } \\
\text { Stimulation }\end{array}$ \\
\hline TV1 & .774 & & & & & \\
\hline TV2 & .684 & & & & & \\
\hline TV3 & .699 & & & & & \\
\hline TV4 & .705 & & & & & \\
\hline TV5 & .613 & & & & & \\
\hline TM1 & & .549 & & & & \\
\hline TM2 & & .703 & & & & \\
\hline TM3 & & .668 & & & & \\
\hline TG1 & & & .619 & & & \\
\hline TG2 & & & .575 & & & \\
\hline TG3 & & & .741 & & & \\
\hline TG4 & & & .673 & & & \\
\hline TP1 & & & & .578 & & \\
\hline TP2 & & & & .704 & & \\
\hline TP3 & & & & .695 & & \\
\hline TS1 & & & & & .838 & \\
\hline TS2 & & & & & .771 & \\
\hline TS3 & & & & & .781 & \\
\hline TS4 & & & & & .801 & \\
\hline TI1 & & & & & & .708 \\
\hline TI2 & & & & & & .769 \\
\hline TI3 & & & & & & .573 \\
\hline TI4 & & & & & & .543 \\
\hline Total Variance Explained & .15 & .14 & .10 & .09 & .09 & .08 \\
\hline Cronbach's Alpha & .86 & .84 & .78 & .70 & .87 & .81 \\
\hline
\end{tabular}

Table 27. Factor Analysis of Trust

\begin{tabular}{ll}
\hline Variable & Factor 1 \\
\hline TRU1 & .865 \\
TRU2 & .877 \\
TRU3 & .860 \\
TRU4 & .883 \\
TRU5 & .902 \\
\hline Total Variance Explained & .38 \\
\hline Cronbach's Alpha & .92 \\
\hline
\end{tabular}


Table 28. Factor Analysis of Harmony

\begin{tabular}{ll}
\hline Variable & Factor 1 \\
\hline HAR1 & .822 \\
HAR2 & .809 \\
HAR3 & .805 \\
HAR4 & .832 \\
HAR5 & .832 \\
\hline Total Variance Explained & .34 \\
\hline Cronbach's Alpha & .87 \\
\hline
\end{tabular}

Table 29. Factor Analysis of Leadership Effectiveness

\begin{tabular}{ll}
\hline Variable & Factor 1 \\
\hline EFF1 & .830 \\
EFF2 & .804 \\
EFF3 & .793 \\
EFF4 & .859 \\
\hline Total Variance Explained & .27 \\
\hline Cronbach's Alpha & .83 \\
\hline
\end{tabular}

Reliability and Validity Tests

Reliability tests were conducted to assess the internal consistency of the measures employed in the study. Reliability refers to the state when a scale yields consistent measures over time (Henson, 2001). Reliability tests provide an important step in instrument validation to ensure measurement accuracy; that is, to minimize the measurement error. Cronbach's alpha $(\alpha)$ is usually calculated to measure reliability, with a commonly used threshold value for acceptable reliability to be .70 (Nunnally, 1978).

Tables 25 to 29 indicate that all the scales have good levels of internal consistency, with the lowest $\alpha$ of .70. The Cronbach's alphas for the three dimensions (moral, benevolent and authoritative leadership) of paternalistic leadership range from .80 to .85 , and the alpha for the full paternalistic leadership scale is .88. The Cronbach's alphas for the six dimensions of transformational leadership range from .70 to .87 , and the alpha for the full transformational 
leadership scale is .93 . The Cronbach's alphas for authoritarian leadership, trust, harmony and effectiveness are $.87, .93, .88$, and .84 , respectively.

To claim the validity of an instrument, it is necessary to check both convergent and discriminant validity. Convergent validity ensures that all items measure a single underlying construct (Hair et al., 1998). Discriminant validity reflects the extent to which measures for each construct are distinctly different from each other (Hair et al., 1998). The test results from Tables 25 to 29 suggest that the subscales within each measure have an adequate level of convergent validity, as all item-total correlations exceed the recommended criterion of .40 and loaded clearly on their associated factors. Discriminant validity for each subscale was achieved because items loaded stronger on their associated factors than on any other factor (Hair et al., 1998).

Intercorrelations between Variables

An analysis of bivariate correlations between all variables was conducted. One of the objectives of this study was to replace authoritarian leadership, one of the dimensions of the paternalistic leadership construct, with authoritative leadership to purge some negative correlations among the three dimensions. The results reveal that different from authoritarian leadership, which negatively correlates with other dimensions of paternalistic leadership and outcomes, authoritative leadership significantly positively correlates with the other two dimensions as well as the outcomes. The intercorrelations between all variables used in this study are reported in table 30. All correlations were significant at $p \leq .01$ level. A potential concern of the correlation tests is the high correlation among the variables. However, high correlation does not necessarily mean that such correlation will suffer from multicollinearity (Parker \& Smith, 1984). Multicollinearity does not affect the estimates in this study as neither the coefficients nor their standard errors demonstrate significant instabilities or fluctuations 
(Parker \& Smith, 1984). In addition, the confirmatory factor analysis and the single-degree-offreedom tests on measurements of paternalistic leadership and transformational leadership reveal that the high correlation is not a problem in this study.

Table 30. Correlations Matrix of all the Variables

\begin{tabular}{|c|c|c|c|c|c|c|c|c|c|}
\hline & PL & TL & TRU & HAR & EFF & ANL & AVL & ML & $\mathrm{BL}$ \\
\hline Paternalistic Leadership (PL) & .88 & & & & & & & & \\
\hline Transformational Leadership (TL) & .85 & .93 & & & & & & & \\
\hline Trust (TRU) & .83 & .67 & .93 & & & & & & \\
\hline Harmony (HAR) & .83 & .71 & .92 & .88 & & & & & \\
\hline Effectiveness (EFF) & .83 & .72 & .90 & .97 & .84 & & & & \\
\hline Authoritarian Leadership (ANL) & -.67 & -.60 & -.56 & -.54 & -.54 & .87 & & & \\
\hline Authoritative Leadership (AVL) & .84 & .66 & .68 & .67 & .66 & -.53 & .80 & & \\
\hline Moral Leadership (ML) & .68 & .66 & .59 & .63 & .63 & -.37 & .40 & .85 & \\
\hline Benevolent Leadership (BL) & .83 & .67 & .68 & .66 & .65 & -.65 & .63 & .25 & .83 \\
\hline
\end{tabular}

Cronbach's alphas are italicized on the diagonal.

Regardless, I further investigated the leadership measures to provide support for the differentiation between paternalistic leadership and transformational leadership. A confirmatory factor analysis was conducted on the items in both scales. The correlations, presented in Table 31, demonstrate that most of the inter-item correlations between subscales from each measure are less than .7, which is frequently accepted as evidence of discriminant validity (Ping, 2005).

Table 31 Correlation Matrix of Paternalistic and Transformational Leadership Items

\begin{tabular}{lccccccccc}
\hline & AVL & ML & BL & TV & TM & TG & TP & TS & TI \\
\hline AVL & 1.00 & & & & & & & & \\
ML & .46 & 1.00 & & & & & & & \\
BL & .77 & .28 & 1.00 & & & & & & \\
TV & .66 & .75 & .63 & 1.00 & & & & & \\
TM & .52 & .58 & .63 & .68 & 1.00 & & & & \\
TG & .62 & .76 & .57 & .75 & .71 & 1.00 & & & \\
TP & .58 & .65 & .42 & .83 & .64 & .69 & 1.00 & & \\
TS & .76 & .34 & .74 & .50 & .61 & .56 & .41 & 1.00 & \\
TI & .59 & .64 & .69 & .69 & .84 & .73 & .59 & .59 & 1.00 \\
\hline AVL: & Authoritative Leadership & & & ML: & Moral Leadership \\
BL: & Benevolent Leadership & & & TV: & Identify and Articulated a Vision \\
TM: & Provide an Appropriate Model & & & TG: & Fostering the Acceptance of Group Goals \\
TP: & High Performance Expectation & & & TS: & Provide Individualized Support \\
TI: & Intellectual Stimulation & & & & &
\end{tabular}


In Table 31, the correlations that are larger than .70 were examined by observing their confidence intervals (CI); neither of them included 1 , which demonstrates discriminant validity (Anderson \& Gerbing, 1988). A single-degree-of-freedom test also was used to compare two measurement models, one with the target correlation fixed at 1 , and a second with this correlation free (Bagozzi \& Phillips, 1982). The difference in resulting chi-squares was significant, which suggests the correlation is not 1 . Thus, paternalistic leadership and transformational leadership are correlationally distinct, which supports discriminant validity.

\section{Control Variables}

Previous research indicates that individual perception of leadership effectiveness differs by gender, educational level, whether the company is state-owned or private, and the geographical location of the company (Farh \& Chen, 2000; Pellegrini \& Scandura, 2008). Therefore, it is important to control for these external factors that might affect the relationships being studied. In all hierarchical regression analyses, dummy coded variables were introduced as control variables in the first step. Gender is scored as 1 for male and 2 for female. Educational level includes five levels: 1 = high school diploma; 2 = vocational/technical certificate; 3 = two-year college degree; 4 = bachelor's degree; and $5=$ master's degree. The nature of the company is categorized into private company (scored as 1) and state-owned company (scored as 2). The respondents are asked to fill in the name of the city in which the company is located. The companies are located in three cities: $1=$ Changsha; $2=$ Xiangxiang; 3 $=$ Hong Kong. 


\section{Hypothesis Testing}

In this section, the statistical analyses used to test the research model and hypotheses are discussed, and the results obtained from the tests are presented. Table 32 includes a summary of the hypotheses proposed in the previous chapter.

Table 32. Summary of Hypotheses

\begin{tabular}{|c|c|}
\hline Models & Hypotheses \\
\hline \multirow{4}{*}{ Mediation Models } & $\begin{array}{l}\text { H1: Subordinates' trust in leader partially mediates the relationship between } \\
\text { paternalistic leadership and its effectiveness on subordinates. }\end{array}$ \\
\hline & $\begin{array}{l}\text { H2: Subordinates' trust in leader partially mediates the relationship between } \\
\text { transformational leadership and its effectiveness on subordinates. }\end{array}$ \\
\hline & $\begin{array}{l}\text { H3: The degree of harmony in the organization partially mediates the } \\
\text { relationship between paternalistic leadership and its effectiveness on } \\
\text { subordinates. }\end{array}$ \\
\hline & $\begin{array}{l}\text { H4: The degree of harmony in the organization partially mediates the } \\
\text { relationship between transformational leadership and its effectiveness on } \\
\text { subordinates. }\end{array}$ \\
\hline \multirow{4}{*}{ Moderation Models } & $\begin{array}{l}\text { H5: Generation moderates the relationship between paternalistic leadership and } \\
\text { subordinates' trust in leader, such that the positive relationship between } \\
\text { paternalistic leadership and trust is stronger for Generation X than for } \\
\text { Generation Y. }\end{array}$ \\
\hline & $\begin{array}{l}\text { H6: Generation moderates the relationship between transformational leadership } \\
\text { and subordinates' trust in leader, such that the positive relationship between } \\
\text { transformational leadership and trust is stronger for Generation Y than for } \\
\text { Generation X. }\end{array}$ \\
\hline & $\begin{array}{l}\text { H7: Generation moderates the relationship between paternalistic leadership and } \\
\text { harmonious relationship between a leader and his/her subordinates, such that } \\
\text { the positive relationship between paternalistic leadership and harmony is } \\
\text { stronger for Generation X than for Generation Y. }\end{array}$ \\
\hline & $\begin{array}{l}\text { H8: Generation moderates the relationship between transformational leadership } \\
\text { and harmonious relationship between a leader and his/her subordinates, such } \\
\text { that the positive relationship between transformational leadership and harmony } \\
\text { is stronger for Generation } Y \text { than for Generation X. }\end{array}$ \\
\hline
\end{tabular}


The processes of proposed mediation hypotheses $\mathrm{H} 1$ to $\mathrm{H} 4$ are presented in Figure 4 in Chapter III. The top panel of Figure 4 represents a causal process in which X, the predictor variable, affects $\mathrm{Y}$, the outcome variable. Path c quantifies this effect, called the total effect of $\mathrm{X}$ on Y. The bottom panel illustrates the components of the total effect. Path a represents the causal effect of the predictor variable on the proposed mediator variable, M. Path b represents the causal effect of the mediator on the outcome variable, controlling for the predictor variable, whereas path c' represents the causal effect of the predictor variable on the outcome variable controlling for the mediator.

To test hypotheses $\mathrm{H} 1$ to $\mathrm{H} 4$, one should demonstrate that 1) the predictor variable predicts the outcome variable; 2) the predictor variable predicts the mediator variable; 3) the mediator variable predicts the dependent variable; and 4) the reduction of the predictor variableoutcome variable regression coefficient after the inclusion of the mediator is significant (Barron \& Kenny, 1986; Frazier et al., 2004). I used the script code written by Preacher and Hayes (2008) and ran analyses from the Macro menu to test all the mediation models.

For Hypothesis 1, the dependent variable/outcome variable is effectiveness, the mediator is trust, and the independent variable/predictor variable is paternalistic leadership. As shown in Table 33, paternalistic leadership was related to effectiveness (path c) as the unstandardized regression coefficient $(B=.41)$ associated with the effect of paternalistic leadership on effectiveness was significant $(\mathrm{p}<.0001)$. The unstandarized regression coefficient $(B=.49)$ associated with the relationship between paternalistic leadership and trust (path a) also was significant $(p<.0001)$. To test whether trust was related to effectiveness (Path $b$ ), effectiveness was regressed simultaneously on both trust and the paternalistic leadership variable. The coefficient associated with the relation between trust and effectiveness (controlling for 
paternalistic leadership) was significant $(B=.51, p<.0001)$. The results show that the relationship between paternalistic leadership and effectiveness (path c') was .16 and still significant $(p<.0001)$, which means that trust partially mediates the relation between paternalistic leadership and effectiveness. The results also indicate that 69.88 percentage of the total effect is mediated. In addition, four control variables, size of the company, gender, location, and whether the company is state-owned or private were significant at $p<.05$, providing support for controlling their influence on effectiveness. To test whether the drop (from $B=.41$ in path c to $B=.16$ in path c') is significant, a Sobel test was used. The Sobel test directly tests for a reduction in the effect of the independent variable on the dependent variable when controlling for the mediator. The result indicates support for the partial mediation hypothesis (Sobel test statistic score $=14.2920, p<.0001)$. Therefore, Hypothesis 1 is supported.

Table 33. Mediating Test for H1

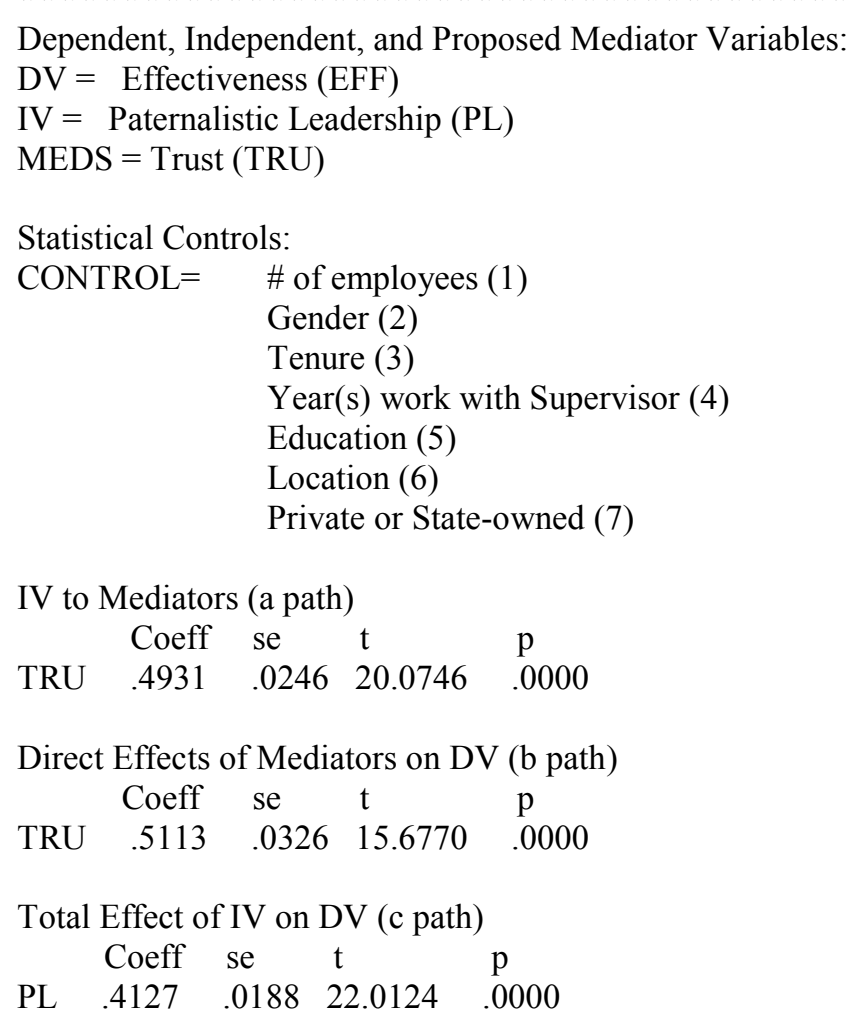


Direct Effect of IV on DV (c-prime path)

$$
\text { Coeff se } \mathrm{t} \quad \mathrm{p}
$$

PL $\quad .1606 \quad .0213 \quad 7.5452 \quad .0000$

Partial Effect of Control Variables on DV

\begin{tabular}{lllll} 
& Coeff & se & \multicolumn{1}{l}{$\mathrm{t}$} & $\mathrm{p}$ \\
$(1)$ & -.0010 & .0002 & -4.5451 & .0000 \\
$(2)$ & .4977 & .2037 & 2.4427 & .0152 \\
$(3)$ & -.0169 & .0239 & -.7063 & .4806 \\
$(4)$ & -.0077 & .0539 & -.1425 & .8868 \\
$(5)$ & -.1759 & .0921 & -1.9092 & .0572 \\
$(6)$ & 1.0169 & .3062 & 3.3214 & .0010 \\
$(7)$ & 1.1603 & .5153 & 2.2516 & .0251
\end{tabular}

Model Summary for DV Model

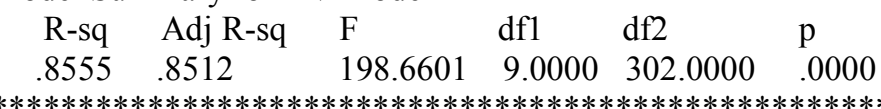

\begin{tabular}{|c|c|c|c|}
\hline Sobel & $P$ value & $\begin{array}{r}\text { Percentage of the } \\
\text { total effect } \\
\text { that is mediated }\end{array}$ & $\begin{array}{l}\text { Ratio of the } \\
\text { indirect to the } \\
\text { direct effect }\end{array}$ \\
\hline 14.291991 & .000000 & 69.881031 & 2.320167 \\
\hline
\end{tabular}

For Hypothesis 2, the dependent variable/outcome variable is effectiveness, the mediator is trust, and the independent variable/predictor variable is transformational leadership. As shown in Table 34, transformational leadership was related to effectiveness (path c) as the unstandardized regression coefficient $(B=.19)$ associated with the effect of transformational leadership on effectiveness was significant $(p<.0001)$. The unstandarized regression coefficient $(B=.20)$ associated with the relationship between transformational leadership and trust (path a) also was significant $(p<.0001)$. To test whether trust was related to effectiveness (Path b), effectiveness was regressed simultaneously on both trust and the transformational leadership variable. The coefficient associated with the relation between trust and effectiveness (controlling for transformational leadership) was significant $(B=.60, p<.0001)$. The results show that the relationship between transformational leadership and effectiveness (path c') was .07 and still significant $(p<.0001)$, which means that trust partially mediates the relation between 
transformational leadership and effectiveness. Results also indicate that 72 percentage of the total effect is mediated. In addition, two control variables, number of employees and whether the company was private or state-owned, were significant at $p<.01$, providing support for controlling their influence on effectiveness. To test whether the drop (from $B=.19$ in path c to $B$ $=.07$ in path c') is significant, a Sobel test was used. The Sobel test directly tests for a reduction in the effect of the independent variable on the dependent variable when controlling for the mediator. The result indicates support for the partial mediation hypothesis (Sobel test statistic score $=13.3885, p<.0001)$. Therefore, Hypothesis 2 is supported.

Table 34. Mediating Test for $\mathrm{H} 2$

Dependent, Independent, and Proposed Mediator Variables:

$\mathrm{DV}=$ Effectiveness $(\mathrm{EFF})$

IV $=$ Transformational Leadership (TL)

MEDS $=$ Trust (TRU)

Statistical Controls:

Sample size: 312

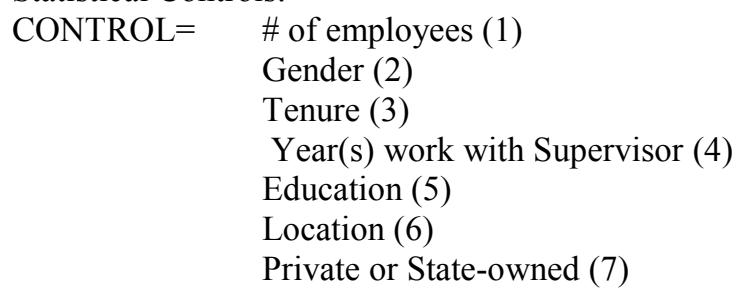




$\begin{array}{lrlrl}(2) & .3299 & .2043 & 1.6150 & .1074 \\ (3) & -.0147 & .0241 & -.6094 & .5427 \\ (4) & -.0345 & .0541 & -.6375 & .5243 \\ (5) & -.1760 & .0930 & -1.8918 & .0595 \\ (6) & .4028 & .2781 & 1.4483 & .1486 \\ (7) & 1.7070 & .5169 & 3.3024 & .0011\end{array}$

Model Summary for DV Model

$\begin{array}{llllll}\text { R-sq } & \text { Adj R-sq } & \text { F } & \text { df1 } & \text { df2 } & \text { p } \\ .8527 & .8483 & 194.2579 & 9.0000 & 302.0000 & .0000\end{array}$

\begin{tabular}{|c|c|c|c|}
\hline Sobel & $P$ value & $\begin{array}{l}\text { Percentage of the } \\
\text { total effect } \\
\text { that is mediated }\end{array}$ & $\begin{array}{l}\text { Ratio of the } \\
\text { indirect to the } \\
\text { direct effect }\end{array}$ \\
\hline 13.388519 & .000000 & 72.001898 & 2.571671 \\
\hline
\end{tabular}

For Hypothesis 3, the dependent variable/outcome variable is effectiveness, the mediator is harmony, and the independent variable/predictor variable is paternalistic leadership. As shown in Table 35, paternalistic leadership was related to effectiveness (path c) as the unstandardized regression coefficient $(B=.41)$ associated with the effect of paternalistic leadership on effectiveness was significant $(p<.0001)$. The unstandarized regression coefficient $(B=.50)$ associated with the relationship between paternalistic leadership and harmony (path a) also was significant $(p<.0001)$. To test whether harmony was related to effectiveness (Path $b$ ), effectiveness was regressed simultaneously on both harmony and the paternalistic leadership variable. The coefficient associated with the relation between harmony and effectiveness (controlling for paternalistic leadership) was significant $(B=.75, p<.0001)$. The results show that the relationship between paternalistic leadership and effectiveness (path c') was .04 and still significant $(p<.005)$, which means that harmony partially mediates the relation between paternalistic leadership and effectiveness. The results also show that two control variables, size of the company and location, were significant at the .05 level and 93.63 percentage of the total effect is mediated. To test whether the drop (from $B=.41$ in path $\mathrm{c}$ to $B=.04$ in path c') is 
significant, a Sobel test was used. The Sobel test directly tests for a reduction in the effect of the independent variable on the dependent variable when controlling for the mediator. The result indicates support for the partial mediation hypothesis (Sobel test statistic score $=21.8230, p<$ .0001). Therefore, Hypothesis 3 is supported.

Table 35. Mediating Test for $\mathrm{H} 3$

Dependent, Independent, and Proposed Mediator Variables:

$\mathrm{DV}=$ Effectiveness (EFF)

$\mathrm{IV}=$ Paternalistic Leadership (PL)

MEDS $=$ Harmony (HAR)

Statistical Controls:

Sample size: 312

CONTROL $=\quad$ \# of employees (1)

Gender (2)

Tenure (3)

Year(s) work with Supervisor (4)

Education (5)

Location (6)

Private or State-owned (7)

IV to Mediators (a path)

Coeff se $\mathrm{t} \quad \mathrm{p}$

$\begin{array}{lllll}\text { HAR } & .4954 & .0224 & 22.1304 & .0000\end{array}$

Direct Effects of Mediators on DV (b path)

$\begin{array}{llll}\text { Coeff se } \mathrm{t} & \mathrm{p}\end{array}$

$\begin{array}{lllll}\text { HAR } & .7484 & .0216 & 34.5750 & .0000\end{array}$

Total Effect of IV on DV (c path)

Coeff se $\mathrm{t} \quad \mathrm{p}$

$\begin{array}{lllll}\text { PL } & .4127 & .0188 & 22.0124 & .0000\end{array}$

Direct Effect of IV on DV (c-prime path)

Coeff se $\mathrm{t} \quad \mathrm{p}$

$\begin{array}{lllll}\text { PL } & .0420 & .0136 & 3.0774 & .0023\end{array}$

Partial Effect of Control Variables on DV

$\begin{array}{lclll} & \text { Coeff } & \mathrm{se} & \mathrm{t} & \mathrm{p} \\ (1) & -.0003 & .0001 & -2.3227 & .0209 \\ (2) & -.1134 & .1248 & -.9087 & .3642 \\ (3) & .0055 & .0143 & .3864 & .6995 \\ (4) & -.0015 & .0326 & -.0469 & .9626 \\ (5) & -.0044 & .0558 & -.0790 & .9371 \\ (6) & .4536 & .1866 & 2.4314 & .0156 \\ (7) & .0989 & .3140 & .3151 & .7529\end{array}$

Model Summary for DV Model

R-sq Adj R-sq $\quad F \quad$ df1 $\quad$ df2 $\quad p$ 


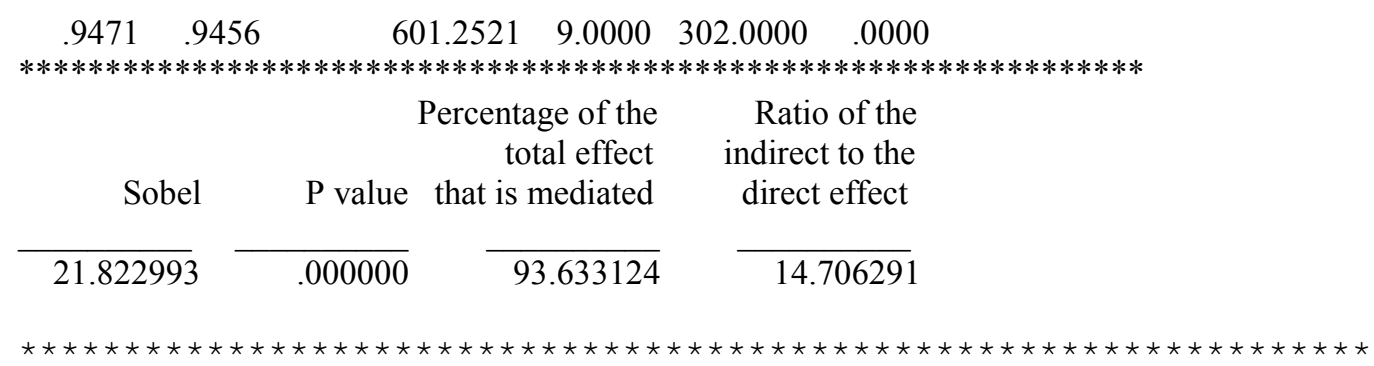

For Hypothesis 4, the dependent variable/outcome variable is effectiveness, the mediator is harmony, and the independent variable/predictor variable is transformational leadership. As shown in Table 36, transformational leadership was related to effectiveness (path c) as the unstandardized regression coefficient $(B=.19)$ associated with the effect of transformational leadership on effectiveness was significant $(p<.0001)$. The unstandarized regression coefficient $(B=.22)$ associated with the relationship between transformational leadership and harmony (path a) also was significant $(p<.0001)$. To test whether harmony was related to effectiveness (Path b), effectiveness was regressed simultaneously on both harmony and the transformational leadership variable. The coefficient associated with the relation between harmony and effectivness (controlling for transformational leadership) was significant $(B=.77, p<.0001)$. The results show that the relationship between transformational leadership and effectiveness (path c') was .02 and still significant $(p<.005)$, which means that harmony partially mediates the relation between transformational leadership and effectiveness. The results also show that the control variable, location, was significant at .05 level and 93.65 percentage of total effect is mediated. To test whether the drop (from $B=.19$ in path c to $B=.02$ in path c') is significant, a Sobel test was used. The Sobel test directly tests for a reduction in the effect of the independent variable on the dependent variable when controlling for the mediator. The result indicates support for the partial mediation hypothesis (Sobel test statistic score $=16.8271, p<.0001$ ). Therefore, Hypothesis 4 is supported. 
Table 36. Mediating Test for H4

Dependent, Independent, and Proposed Mediator Variables:

$\mathrm{DV}=$ Effectiveness $(\mathrm{EFF})$

$\mathrm{IV}=$ Transformational Leadership (TL)

MEDS $=$ Harmony $($ TRU $)$

Statistical Controls:

Sample size: 312

$\begin{aligned} \text { CONTROL }= & \text { \# of employees }(1) \\ & \text { Gender (2) } \\ & \text { Tenure (3) } \\ & \text { Year(s) work with Supervisor (4) } \\ & \text { Education (5) } \\ & \text { Location (6) } \\ & \text { Private or State-owned (7) }\end{aligned}$

IV to Mediators (a path)

$\begin{array}{lllll} & \text { Coeff } & \mathrm{se} & \mathrm{t} & \mathrm{p} \\ \text { HAR } & .2192 & .0158 & 13.8474 & .0000\end{array}$

Direct Effects of Mediators on DV (b path)

Coeff se $t$ p

$\begin{array}{lllll}\text { HAR } & .7718 & .0172 & 44.9806 & .0000\end{array}$

Total Effect of IV on DV (c path)

$\begin{array}{llll}\text { Coeff } & \text { se } & \mathrm{p}\end{array}$

$\begin{array}{lllll}\text { TL } & .1856 & .0131 & 14.1672 & .0000\end{array}$

Direct Effect of IV on DV (c-prime path)

Coeff se $\mathrm{t}$ p

$\begin{array}{lllll}\text { TL } & .0164 & .0060 & 2.7106 & .0071\end{array}$

Partial Effect of Control Variables on DV

$\begin{array}{lcccl} & \text { Coeff } & \mathrm{se} & \mathrm{t} & \mathrm{p} \\ \text { (1) } & -.0003 & .0001 & -1.9996 & .0464 \\ (2) & -.1761 & .1224 & -1.4393 & .1511 \\ (3) & .0055 & .0145 & .3780 & .7057 \\ (4) & -.0081 & .0326 & -.2479 & .8044 \\ (5) & .0015 & .0559 & .0270 & .9785 \\ (6) & .2687 & .1647 & 1.6316 & .1038 \\ \text { (7) } & .2144 & .3162 & .6781 & .4982\end{array}$

Model Summary for DV Model

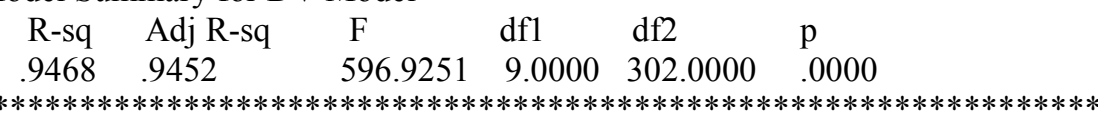

Percentage of the Ratio of the total effect indirect to the

Sobel P value that is mediated direct effect

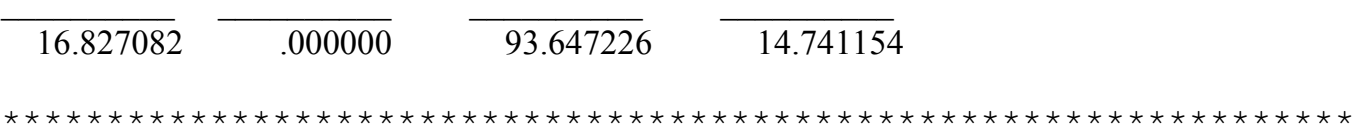


As discussed in the previous chapter, there is a possibility that people from different generations may have different perspectives on leadership effectiveness. Therefore, the moderation models were proposed and hypotheses 5 to 8 were developed to test the moderating effect of generation using hierarchical moderated multiple regression (HMMR). Before conducting the analyses, generation was dummy coded with Generation X coded as 1 and Generation $\mathrm{Y}$ coded as 0 . Next, I centered the independent variables (i.e., paternalistic leadership, transformational leadership) to reduce problems associated with multicollinearity among the variables in the regression (Cohen et al., 2003). The centered predictor is then multiplied with the coded moderator variable to create the product term representing the interaction between the predictor and moderator. The hierarchical regression consists of three steps: 1) enter the control variables in Block 1 of 3;2) enter the main effect variables, paternalistic leadership and transformational leadership, and moderator variable (generation) in block 2 of $3 ; 3$ ) add the interaction term in Block 3 of 3 . The change in $R^{2}$ between step 2 and step 3 was examined to determine whether the moderator was significant. If the $\mathrm{R}^{2}$ change is statistically significant after the inclusion of the interaction term, this indicates that there is a statistically significant moderator effect.

Hypothesis 5 states that generation moderates the relationship between paternalistic leadership and subordinates' trust in leader, such that the positive relationship between paternalistic leadership and trust is stronger for Generation X than for Generation Y. Looking at the output in Table 37, the results indicate that all the control variables were non-significant (step 1); thus, these factors did not influence the model. In step 2, paternalistic leadership significantly and positively predicted trust $(\beta=.74, p<.0001)$. In addition, there is a main effect for generation $(\beta=-.39, p<.0001)$; those in Generation $Y$ trusted their leaders more than those in 
Generation X (mean for Generation $Y=24.69$, mean for Generation $X=21.40$ ). The most important finding was the result for the interaction term (step 3), which was a significant predictor of trust $(\beta=.21, p<.0001)$, and which significantly increased $\mathrm{R}^{2}\left(\Delta \mathrm{R}^{2}=.03, p<\right.$ .0001). In order to determine whether the significant moderator effect was in the predicted direction, results were graphed and are shown in Figure 9. Observation of the slopes of the lines for both Generation X and Generation Y reveals that employees from Generation X do tend to increase their perceptions of trust at a greater rate as paternalistic leadership increases than those from Generation Y. The increase in trust for Generation Y was not as great. The results of the analyses and the graphic depiction both support hypothesis 5 .

\section{Table 37. Moderation Test for H5}

\begin{tabular}{|c|c|c|c|c|}
\hline Steps and variable & B & $\beta$ & $\mathrm{R}^{2}$ & $\Delta \mathrm{R}^{2}$ \\
\hline \multicolumn{5}{|c|}{ Dummy coding (Generation X coded 1 , Generation $Y$ coded 0 ) } \\
\hline Step 1 & & & & \\
\hline Number of Employees & .00 & -.10 & & \\
\hline Gender & -.20 & -.02 & & \\
\hline Tenure & .01 & -.01 & & \\
\hline Years Work with Supervisor & -.01 & -.01 & & \\
\hline Education & .21 & .06 & & \\
\hline Location & -.57 & -.06 & & \\
\hline Property & .87 & .10 & & \\
\hline Step 2 & & & $.76 * *$ & \\
\hline Paternalistic Leadership & 7.30 & $.74 * *$ & & \\
\hline Generation & -3.38 & $-.39 * *$ & & \\
\hline Step 3 & & & $.79 * *$ & $.03 * *$ \\
\hline Paternalistic Leadership & 6.20 & $.63 * *$ & & \\
\hline Generation & -4.12 & $-.48 * *$ & & \\
\hline Paternalistic Leadership $\times$ Generation & 3.43 & $.21 * *$ & & \\
\hline
\end{tabular}


Figure 9. Moderator Effect of Generation on the Paternalistic Leadership - Trust Relationship Moderator Effect of Generation on the Paternalistic Leadership-Trust Relationship
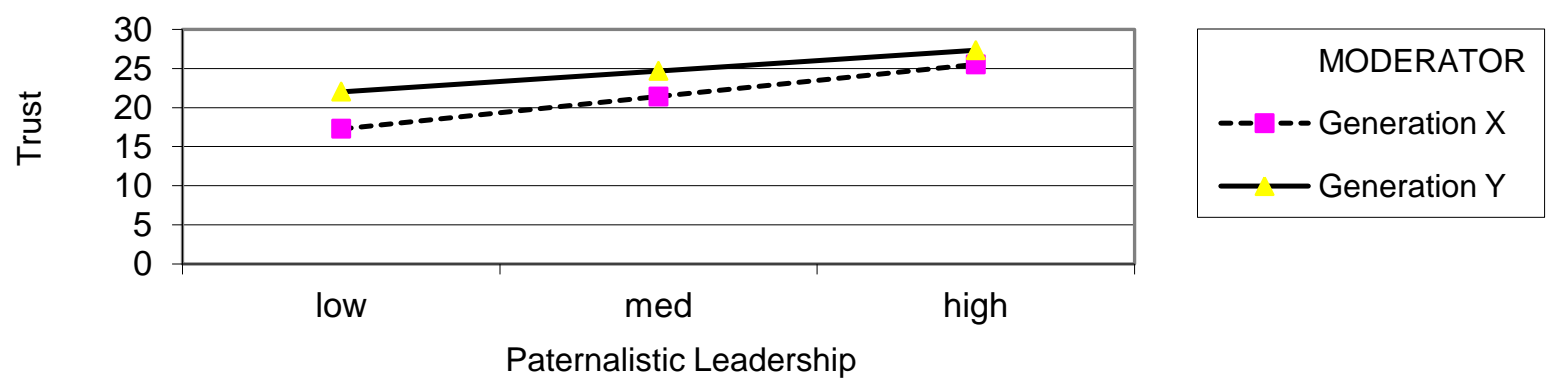

Hypothesis 6 states that generation moderates the relationship between transformational leadership and subordinates' trust in leader, such that the positive relationship between transformational leadership and trust is stronger for Generation $\mathrm{Y}$ than for Generation $\mathrm{X}$. Looking at the output in Table 38, the results indicate that except location and property of the company (whether the company is private or state-owned), other control variables are not significantly related to trust (step 1). In step 2, transformational leadership significantly and positively predicted trust $(\beta=.29, p<.0001)$. In addition, there is a main effect for generation $(\beta$ $=-.46, p<.0001)$; those in Generation $\mathrm{Y}$ trusted their leader more than those in Generation $\mathrm{X}$ (mean for Generation $Y=25.09$, mean for Generation $X=21.09$ ). The most important finding was the result for the interaction term (step 3), which was a significant predictor of trust $(\beta=$ $.18, p<.01)$, and which significantly increased $\mathrm{R}^{2}\left(\Delta \mathrm{R}^{2}=.02, p<.01\right)$. In order to determine whether the significant moderator effect was in the predicted direction, results were graphed and are shown in Figure 10. Observation of the slopes of the lines for both Generation $\mathrm{X}$ and Generation Y reveal that employees from Generation Y do tend to increase their perceptions of trust at a greater rate as transformational leadership increases than those from Generation X. The 
increase in trust for Generation $\mathrm{X}$ was not as great. The results of the analyses and the graphic depiction both support hypothesis 6 .

Table 38. Moderation Test for H6

\begin{tabular}{|c|c|c|c|c|}
\hline Steps and variable & B & $\beta$ & $\mathrm{R}^{2}$ & $\Delta \mathrm{R}^{2}$ \\
\hline \multicolumn{5}{|c|}{ Dummy coding (Generation X coded 1 , Generation Y coded 0 ) } \\
\hline Step 1 & & & & \\
\hline Number of Employees & .01 & .16 & & \\
\hline Gender & -.70 & -.08 & & \\
\hline Tenure & -.12 & -.18 & & \\
\hline Years Work with Supervisor & -.01 & -.01 & & \\
\hline Education & .10 & .01 & & \\
\hline Location & -5.70 & $-.62 *$ & & \\
\hline Property & 4.53 & $.51 *$ & & \\
\hline$\underline{\text { Step } 2}$ & & & $.32 * *$ & \\
\hline Transformational Leadership & 2.38 & $.29 * *$ & & \\
\hline Generation & -3.97 & $-.46^{* *}$ & & \\
\hline Step 3 & & & $.34 * *$ & $.02 *$ \\
\hline Transformational Leadership & 3.24 & $.40 * *$ & & \\
\hline Generation & -3.50 & $-.41 * *$ & & \\
\hline Transformational Leadership $x$ Generation & -2.19 & $-.18^{*}$ & & \\
\hline
\end{tabular}

${ }^{* *} p<.0001,{ }^{*} \mathrm{p}<.01$

Figure 10. Moderator Effect of Generation on the Transformational Leadership -Trust Relationship

\section{Moderator Effect of Generation on the Transformational Leadership-Trust Relationship}

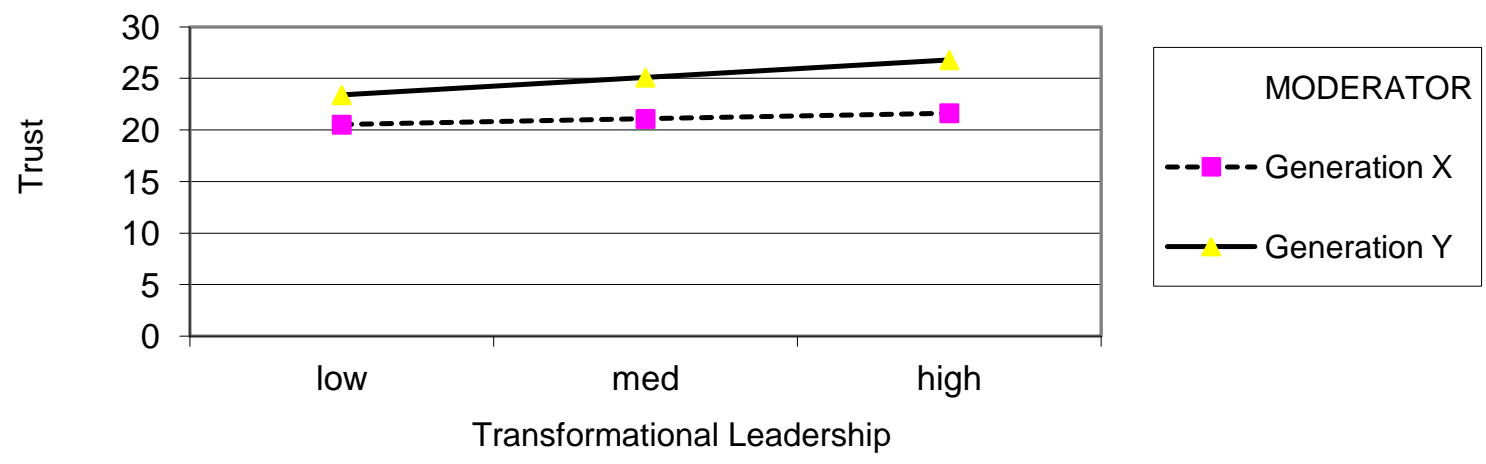

Hypothesis 7 states that generation moderates the relationship between paternalistic

leadership and harmony between a leader and his/her subordinates, such that the positive relationship between paternalistic leadership and harmony is stronger for Generation $\mathrm{X}$ than for 
Generation Y. Looking at the output in Table 39, the results indicate that all the control variables were non-significant (step 1); thus, these factors did not influence the model. In step 2, paternalistic leadership significantly and positively predicted harmony $(\beta=.72, p<.0001)$. In addition, there is a main effect for generation $(\beta=-.38, p<.0001)$; those in Generation $\mathrm{Y}$ experienced more harmony than those in Generation $\mathrm{X}$ (mean for Generation $\mathrm{Y}=25.14$, mean for Generation $X=22.23$ ). The most important finding was the result for the interaction term (step 3), which was a significant predictor of harmony $(\beta=.28, p<.0001)$, and which significantly increased $\mathrm{R}^{2}\left(\Delta \mathrm{R}^{2}=.04, p<.0001\right)$. In order to determine whether the significant moderator effect was in the predicted direction, results were graphed and are shown in Figure 11. Observation of the slopes of the lines for both Generation X and Generation Y reveal that employees from Generation $\mathrm{X}$ do tend to increase their perceptions of harmony at a greater rate as paternalistic leadership increases than those from Generation Y. The increase in harmony for Generation Y was not as great. The results of the analyses and the graphic depiction both support hypothesis 7 .

Table 39. Moderation Test for H7

\begin{tabular}{|c|c|c|c|c|}
\hline Steps and variable & $\mathrm{B}$ & $\beta$ & $\mathrm{R}^{2}$ & $\Delta \mathrm{R}^{2}$ \\
\hline \multicolumn{5}{|c|}{ Dummy coding (Generation $X$ coded 1 , Generation $Y$ coded 0 ) } \\
\hline$\underline{\text { Step } 1}$ & & & & \\
\hline Number of Employees & -.00 & -.30 & & \\
\hline Gender & .61 & .07 & & \\
\hline Tenure & .04 & .06 & & \\
\hline Years Work with Supervisor & .01 & .00 & & \\
\hline Education & -.04 & -.01 & & \\
\hline Location & .67 & .08 & & \\
\hline Property & 1.46 & .18 & & \\
\hline Step 2 & & & $.73 * *$ & \\
\hline Paternalistic Leadership & 6.70 & $.72 * *$ & & \\
\hline Generation & -3.02 & $-.38 * *$ & & \\
\hline Step 3 & & & $.77 * *$ & $.04 * *$ \\
\hline Paternalistic Leadership & 5.31 & $.57 * *$ & & \\
\hline Generation & -3.97 & $-.50 * *$ & & \\
\hline Paternalistic Leadership $x$ Generation & 4.35 & $.28 * *$ & & \\
\hline
\end{tabular}


Figure 11. Moderator Effect of Generation on the Paternalistic Leadership -Trust Relationship

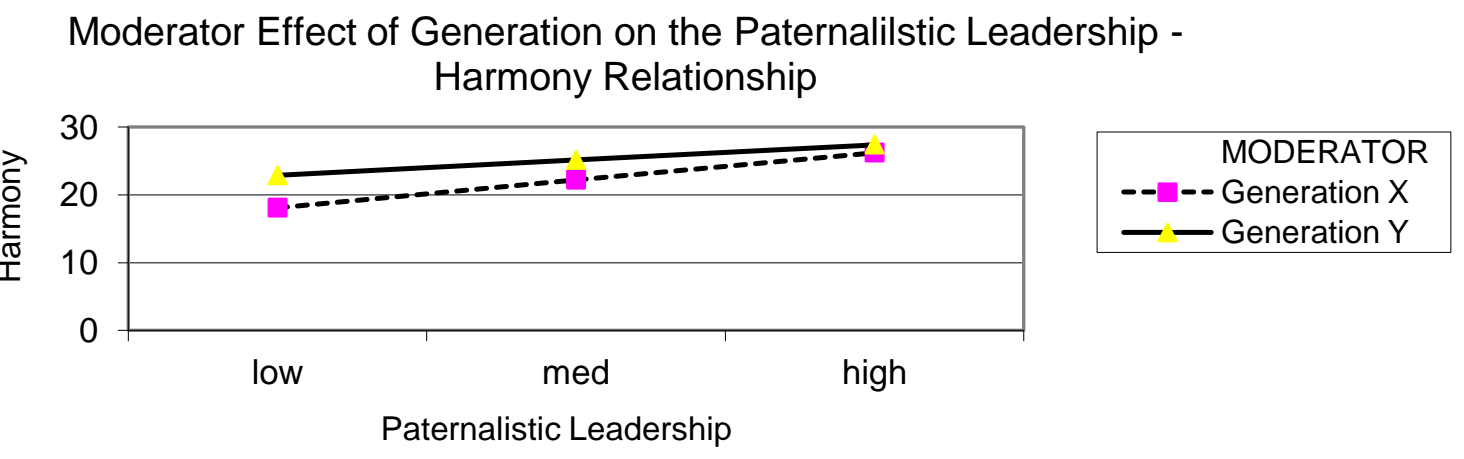

Hypothesis 8 states that generation moderates the relationship between transformational leadership and harmony between a leader and his/her subordinates, such that the positive relationship between transformational leadership and harmony is stronger for Generation $\mathrm{Y}$ than for Generation X. Looking at the output in Table 40, the results indicate that all control variables except property were non-significant (step 1); which shows most factors did not influence the model. In step 2 , transformational leadership significantly and positively predicted harmony ( $\beta=$ $.34, p<.0001)$. In addition, there is a main effect for generation $(\beta=-.44, p<.0001)$; those in Generation $\mathrm{Y}$ experienced more harmony than those in Generation $\mathrm{X}$ (mean for Generation $\mathrm{Y}=$ 25.27, mean for Generation $X=21.69$ ). The most important finding was the result for the interaction term (step 3 ), which was a significant predictor of harmony $(\beta=-.25, p<.0001)$, and which significantly increased $\mathrm{R}^{2}\left(\Delta \mathrm{R}^{2}=.04, p<.0001\right)$. In order to determine whether the significant moderator effect was in the predicted direction, results were graphed and are shown in Figure 12. Observation of the slopes of the lines for both Generation $\mathrm{X}$ and Generation $\mathrm{Y}$ reveal that employees from Generation $\mathrm{Y}$ do tend to increase their perceptions of harmony at a greater rate as transformational leadership increases than those from Generation X. The increase in harmony for Generation X was not as great. The results of the analyses and the graphic depiction both support hypothesis 8 . 
Table 40. Moderation Test for $\mathrm{H} 8$

\begin{tabular}{|c|c|c|c|c|}
\hline Steps and variable & $\mathrm{B}$ & $\beta$ & $\mathrm{R}^{2}$ & $\Delta \mathrm{R}^{2}$ \\
\hline \multirow{2}{*}{\multicolumn{5}{|c|}{ Dummy coding (Generation X coded 1 , Generation $Y$ coded 0 ) }} \\
\hline & & & & \\
\hline Number of Employees & .00 & -.10 & & \\
\hline Gender & .17 & .02 & & \\
\hline Tenure & -.10 & -.15 & & \\
\hline Years Work with Supervisor & .02 & .02 & & \\
\hline Education & -.15 & -.05 & & \\
\hline Location & -3.91 & -.45 & & \\
\hline Property & 4.98 & $.60^{*}$ & & \\
\hline Step 2 & & & $.33 * *$ & \\
\hline Transformational Leadership & 2.59 & $.34 * *$ & & \\
\hline Generation & -3.54 & $-.44 * *$ & & \\
\hline$\underline{\text { Step } 3}$ & & & $.37 * *$ & $.04 * *$ \\
\hline Transformational Leadership & 3.73 & $.49^{* *}$ & & \\
\hline Generation & -2.92 & $-.37 * *$ & & \\
\hline Transformational Leadership $\times$ Generation & -2.88 & $-.25^{* *}$ & & \\
\hline
\end{tabular}

${ }^{* *} p<.0001,{ }^{*} \mathrm{p}<.01$

Figure 12. Moderator Effect of Generation on the Transformational Leadership -Harmony Relationship

Moderator Effect of Generation on the Transformational Leadership-Harmony Relationship

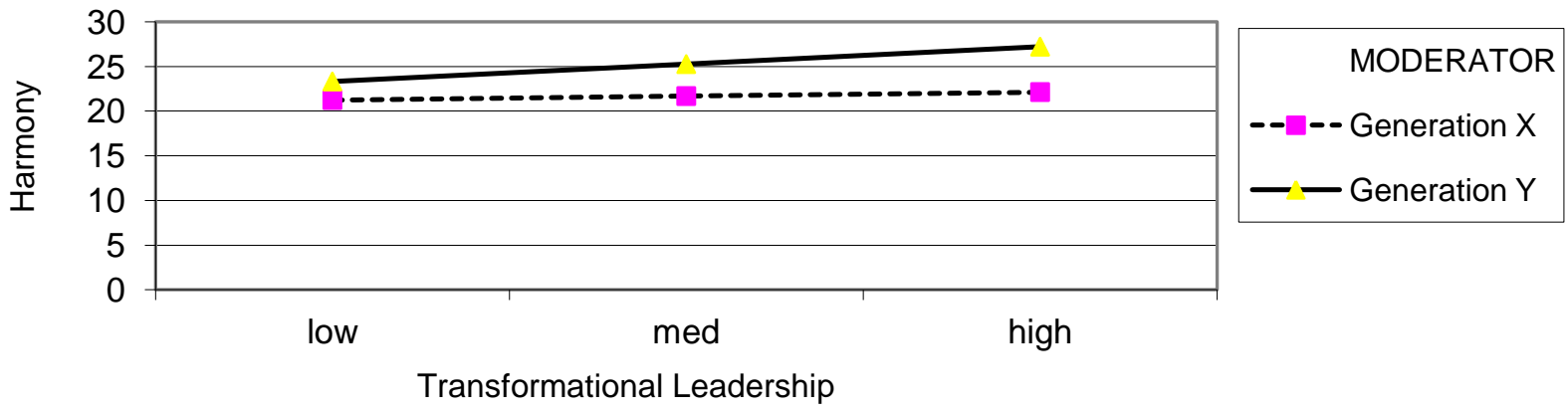

Summary

Results of factor and regression analyses to test the hypotheses were presented in this chapter. The core of this empirical study was to examine the effects of factors such as trust, harmony, and generation on relationships between leadership style and effectiveness. Further, I sought to develop a measurement instrument for harmony and to refine a measurement instrument for paternalistic leadership by replacing authoritarian leadership with authoritative 
leadership. Factor analysis results supported the presence of variables with the proposed dimensions. Thus, this study provides newly developed instruments to measure paternalistic leadership and harmony with acceptable validity and reliability.

Regression results suggested both trust and harmony partially mediate the relationship between leadership style (paternalistic leadership and transformational leadership) and effectiveness. Furthermore, generation was found to moderate the relationship between leadership style and both trust and harmony. All proposed hypotheses were supported and results were presented in both tabular and graphical forms. Table 41 provides the summary of results for the hypotheses. The discussion along with implications of these findings are addressed in the next chapter.

Table 41. Summary of Hypotheses Testing

\begin{tabular}{c|l|c}
\hline \multicolumn{1}{c|}{ Models } & \multicolumn{1}{c}{ Hypotheses } & \multicolumn{1}{c}{ Results } \\
\hline Mediation Models & $\begin{array}{l}\text { H1: Subordinates' trust in leader partially mediates the } \\
\text { relationship between paternalistic leadership and its } \\
\text { effectiveness on subordinates. }\end{array}$ & $\begin{array}{l}\text { H2: Subordinates' trust in leader partially mediates the } \\
\text { relationship between transformational leadership and its } \\
\text { effectiveness on subordinates. }\end{array}$ \\
$\begin{array}{l}\text { H3: The degree of harmony in the organization partially } \\
\text { mediates the relationship between paternalistic leadership } \\
\text { and its effectiveness on subordinates. }\end{array}$ & $\begin{array}{l}\text { S4: The degree of harmony in the organization partially } \\
\text { mediates the relationship between transformational } \\
\text { leadership and its effectiveness on subordinates. }\end{array}$ \\
\hline $\begin{array}{l}\text { H5: Generation moderates the relationship between } \\
\text { paternalistic leadership and subordinates' trust in leader, } \\
\text { such that the positive relationship between paternalistic } \\
\text { leadership and trust is stronger for Generation X than for } \\
\text { Generation Y. }\end{array}$ & $\begin{array}{l}\text { Supported } \\
\text { H6: Generation moderates the relationship between } \\
\text { transformational leadership and subordinates' trust in leader, } \\
\text { such that the positive relationship between transformational }\end{array}$ & Supported \\
\hline
\end{tabular}




\begin{tabular}{l|l|c}
\hline Moderation Models & $\begin{array}{l}\text { leadership and trust is stronger for Generation Y than for } \\
\text { Generation X. }\end{array}$ & $\begin{array}{l}\text { H7: Generation moderates the relationship between } \\
\text { paternalistic leadership and harmonious relationship } \\
\text { between a leader and his/her subordinates, such that the } \\
\text { positive relationship between paternalistic leadership and } \\
\text { harmony is stronger for Generation X than for Generation Y. }\end{array}$ \\
$\begin{array}{l}\text { H8: Generation moderates the relationship between } \\
\text { transformational leadership and harmonious relationship } \\
\text { between a leader and his/her subordinates, such that the } \\
\text { positive relationship between transformational leadership } \\
\text { and harmony is stronger for Generation Y than for } \\
\text { Generation X. }\end{array}$ & Supported \\
\hline
\end{tabular}




\section{CHAPTER V}

\section{DISCUSSION}

The preceding chapter reported the results of the data analysis and hypothesis testing for this study. The discussion in this chapter extends the data analysis to glean both descriptive and normative implications for advancing leadership theories and practices. Toward that goal, findings for each hypothesized relationship included within the research model are interpreted. The chapter is divided into three parts. First, the implications of the findings are addressed. Second, the limitations of the study are presented. Finally, given the results of this study, future research is suggested.

\section{Implications of the Findings}

The major purpose of this study is to introduce, explain, and develop theory that incorporates Chinese leadership philosophies. At a time when China is becoming more central in a globalizing world economy, business managers as well as scholars from outside China increasingly feel the importance of understanding the views and perspectives of Chinese leadership and management. There is little in the large amount of published literature on leadership that is specifically focused on Chinese leadership. In this study, I developed a model that incorporates Chinese business leaders' management philosophies, in which two main leadership styles, paternalistic leadership and transformational leadership, were examined. Particularly, the mediating effects of trust and harmony were tested. Moreover, the moderating effect of generation was examined; that is, I focused on how people from different generations view leaders differently. The following sections discuss the results of measurement instrument development and hypothesis testing concluding with possible explanations for the findings. 


\section{Measurement Instrument Development}

One of the primary focuses of this study was to develop the theoretical underpinnings and provide measurement instruments for paternalistic leadership and harmony. This goal was successfully achieved. The three-domain model of paternalistic leadership (authoritarianism, benevolence, and morality) proposed by Farh and Cheng (2000) has been widely used in empirical studies; however, researchers report concerns on the negative inter-dimensional correlations among the three domains of paternalistic leadership (Pellegrini \& Scandura, 2008; Aycan, 2006). Furthermore, benevolence and morality have been found to be positively related with outcomes such as loyalty to the leaders and subordinates' identification with them, whereas authoritarianism has been found to be negatively associated with those outcomes (Cheng et al., 2004). In addition, the original paternalistic leadership model was introduced based on case studies of the management styles of owners/managers of overseas Chinese family businesses from the 1960s to the 1980s (Farh \& Cheng, 2000). With rapid societal modernization, family businesses no longer play such a dominant role in the Chinese economy, and the social values also are changing. All these issues call for a re-examination of the construct domain of paternalistic leadership to render it compatible with the contemporary period.

In this study, paternalistic leadership is reinterpreted as a leadership style consisting of authoritative, moral, and benevolent leadership. Authoritative leadership replaces the previous dimension of authoritarian leadership. The exploratory factor analysis results confirmed the revision to the construct of paternalistic leadership as all the items clearly loaded onto three factors. Moreover, the internal consistency reliability of all three subscales (authoritative, moral, and benevolent leadership) exceeded .80 and provided a good level of discriminant validity. The new construct of paternalistic leadership successfully achieved a positive alignment and coherence among the three dimensions and purged the negative correlations between 
authoritarian leadership and outcomes. Different from authoritarian leadership, which negatively correlates with other dimensions of paternalistic leadership $(r=-.37$ with moral leadership, $r=$ -.65 with benevolent leadership) and outcomes $(r=-.56$ with trust, $r=-.54$ with harmony, and $r=$ -.54 with effectiveness), authoritative leadership significantly positively correlates with the other two paternalistic dimensions ( $\mathrm{r}=.40$ with moral leadership, $\mathrm{r}=.63$ with benevolent leadership) as well as the outcomes ( $\mathrm{r}=.68$ with trust, $\mathrm{r}=.67$ with both harmony and effectiveness). These results indicate that paternalistic leadership can be treated as one construct rather than three separate sub-constructs.

In China, harmony is precious because it means there is cohesion in the organization (Westwood, 1997). Both internal and external harmony help firms avoid conflict and reduce management costs so that the companies can run smoothly and make profits (Westwood, 1997; Gallo, 2008). Researchers have found that having a Chinese organization that is in harmony and is balanced is important (Gallo, 2008; Zhang et al., 2008). To achieve a harmonious relationship within an organization, a leader should balance all forces within the organization appropriately, weigh the consequences of the extreme positions in an argument and identify the right solution from competing or conflicting perspectives, and maintain flexibility in managerial activities. Although the importance of harmony is recognized, this construct is greatly understudied. One key purpose of this study was to explore the implications of harmony, requiring the development of a valid, reliable harmony scale that could be used in later quantitative studies.

The construct of harmony was developed based on the Confucian view. The concept was interpreted as harmony in order, the manifestation of the right relationships, and providing the practical conditions for the actualization of the right things in the organization. Factor analysis results for the harmony scale provided support for the measure. Item loadings confirmed that the 
proposed items loaded together on one factor and there were no cross loadings. Further, reliability analysis for harmony showed an acceptable level for Cronbach's alpha $(\mathrm{a}=.88)$. Results for hypotheses discussed in a later section of this chapter provide support for the construct validity of the scale.

\section{The Effect of Trust}

From the employees' perspective, trust in the leader means faith in and loyalty to the leader. Previous research has found the mediating effect of trust on the relationship between leadership and performance. The results of this study demonstrate the mediating role played by subordinates' trust in the transformational leadership process. More importantly, results also suggest that trust is an important explanatory mechanism for the relationship between paternalistic leadership and employee performance - a relationship that has not received much attention (Pellegrini \& Scandura, 2008). The findings suggest that subordinates' trust is critical for both transformational and paternalistic leaders to be perceived as effective leaders. Results reveal that the partial mediating effect was slightly stronger in the transformational leadership process ( $72 \%$ mediation) than in the paternalistic leadership process $(69.9 \%$ mediation). A potential explanation for this finding may be that trust has more impact on the effectiveness of transformational leaders because they greatly rely on mutual trust and subordinates are empowered to accomplish organizational goals in a relatively automatic manner; whereas, paternalistic leaders more prefer subordinates to follow their instruction regardless of their level of trust in their leaders.

A plausible explanation regarding the significant role of trust as a mediator in the relationship between leadership styles and effectiveness may be related to the nature of the Chinese context. In this study, trust is measured by asking the respondents whether they feel a 
strong loyalty to their supervisors, whether they support and have complete faith in the integrity of their supervisors. The results show that whether under a paternalistic or transformational leader, trust is a key mechanism that creates loyalty to management by the employees and builds a good relationship between managers and employees. In China, trust is established based on the personal bond and sharing of positive affect between managers and employees (Chen et al., 2011). This trust signals a strong sense of sharing within the relationship where the employees are willing to express their ideas and concerns without fears of being reprimanded. This positive relationship induces positive emotional feelings in their leader by the employees and, therefore, taps into positive evaluations about the effectiveness of their leader.

\section{The Effect of Harmony}

The notion of harmony was most frequently cited by researchers (Westwood, 1997; Silin, 1976; Aycan et al., 2000) in their business studies in China. Based on the Confucian view, harmony was interpreted in this study to mean keeping good institutional order, maintaining proper social relationships, and discovering and following the correct way to make the concrete rules in order to maintain right relationships. The results of the hypothesis tests reveal that the degree of harmony in the organization partially mediates the relationship between both paternalistic and transformational leadership and their effectiveness. Practically speaking, harmony virtually fully mediates the relationship between both leadership styles and effectiveness.

An explanation of the strong mediating effect of harmony on the relationships between both leadership styles and effectiveness may be due to the importance of the Chinese context within which leadership was enacted. Harmony is not a central construct in Western ideology where basic value orientations are based upon notions of equality, egalitarianism and consensus 
(Westwood, 1997). It is, however, a deeply embedded social value in Chinese society, where tradition stresses harmony between people and their environment, and in social relationships. In China, harmony is accomplished through following the rules of proper behavior with the status hierarchy. An effective Chinese leader simulates family patriarchy, and the authority and power is accepted as natural, proper, absolute, and unchallengeable. The significance of harmony in the Chinese culture perhaps explains why harmony has such a strong mediating effect.

The results reveal that to be an effective leader in China one should not only exhibit either transformational or paternalistic leadership, but the leader also must meet the requirements for social harmony. Chinese people are deeply rooted in the traditional Confucian ethic that emphasizes clear hierarchical relationship structures and respect for authority. People are obligated to accept their roles and positions and not to threaten or challenge the structural arrangements. These socialized values are quite different from the Western mindset where socialization practices emphasize independence, questioning and individualism (Yang, 1986). The roles of father and patriarch have absolute social legitimacy within Confucian traditions and by extension, the managers and leaders of organizations have the same kind of legitimized rights and authority. The acceptance of socially approved rules of behavior based on ordered hierarchy creates a basis for the requirement of harmony.

Harmony helps foster more solidaristic sentiments and consolidates beneficial bonds of mutual obligation and reciprocity between leaders and subordinates. The exhibition of proper and considerate behavior by the leader provides valuable support to the maintenance of order and compliance. Authority and hierarchical structures are supported by displays of proper behavior, considerateness, and the pursuit of harmony. Role adherence, conformity to hierarchical order, and maintaining proper social relationships are essential in the creation and sustaining of a 
harmonious system, and in attaining success as a leader within an organization in China, regardless which style of leadership is used.

\section{The Role of Generation}

Perspectives of leadership in China were obtained from two generations. Hypothesis 5 states that generation moderates the relationship between paternalistic leadership and subordinates' trust in leader, such that the positive relationship between paternalistic leadership and trust is stronger for Generation X than for Generation Y. Hypothesis 6 states that generation also moderates the relationship between transformational leadership and subordinates' trust in leader, such that the positive relationship between transformational leadership and trust is stronger for Generation Y than for Generation X. Hypothesis 7 states that generation moderates the relationship between paternalistic leadership and the harmonious relationship between a leader and his/her subordinates, such that the positive relationship between paternalistic leadership and harmony is stronger for Generation $\mathrm{X}$ than for Generation Y. Hypothesis 8 states that generation also moderates the relationship between transformational leadership and the harmonious relationship between a leader and his/her subordinates, such that the positive relationship between transformational leadership and harmony is stronger for Generation $\mathrm{Y}$ than for Generation X. Results confirmed the moderating effect of generation on the relationship between leadership styles and trust in leaders as well as the harmonious relationship within the organization. That is, under a paternalistic leader, employees in Generation X (the older generation) trust more in their leader and experience a more harmonious relationship within the organization than employees in Generation Y (the younger generation). In contrast, employees in Generation Y working for transformational leaders perceive more trust and harmony in the organization than do those in Generation X. 
These findings reveal that in the case of paternalistic leadership, the traditional root of respect for the authority associated with hierarchical positions, which is reflected differently between the two generations, impacts the trust in the leader. Under paternalistic leadership, a significant social gap is maintained between the subordinates and their leader; this gap is seen as a necessary part of the relationship and is expected by Generation X as people from Generation $X$ are found to be oriented toward more traditional values (Zhang \& Sharitt, 2003). Compared with the younger generation, Generation X views paternalistic leadership with widely unequal power distributions to be natural and proper. People from Generation X provide loyalty, support, and cooperation to reciprocate their leader's benevolent care and their trust in their leaders is higher. These are deep-rooted traditional values that sustain this orientation.

Individuals in Generation Y grew up with more personal rights and freedom than the previous generations. The explanations for the moderating effect of generation on the relationship between transformational leadership and trust/harmony may be more complex. For Generation Y, the flood of new information, academic opportunities, and world knowledge has been highly appealing and has shaped a life-long inclination toward learning from multiple sources. People from Generation Y are balanced between the Chinese traditional heritage and exploration of opportunities in the West. They are better educated and more open-minded than previous generations. Also, they are active thinkers, embracing various challenges and new ideas (Zhao \& Liu, 2006). For Generation Y, the good boss is one who stays in the background and allows subordinates to figure out their own best way to do things (Stanat, 2005). Different from Generation X, who is expected to be told what to do, Generation Y views transformational leaders more positively and trusts them more. Transformational leaders delegate responsibility and authority to followers, thus empowering them to accomplish organizational goals in a 
relatively autonomous manner. This approach is perceived by Generation $\mathrm{Y}$ as providing an ideal and harmonious working environment. Individuals in Generation $\mathrm{Y}$ value forward thinking and forward looking behavior and have high achievement needs. These values are compatible with transformational leadership, which deals with creating and building commitment toward a vision for the future of the unit or organization.

The results not only reveal that people from different generations have different perspectives about leadership, but they also reveal that different generations have different understandings about trust in others and harmonious relationships between them. Individuals in Generation Y scored higher in trust and harmony than did those from Generation X. The finding is consistent with the characteristics of Generation Y, with members who are more confident, open, and positive oriented. The results help to explain the changes between different generations as China develops. The younger generation is more open, aggressive, self-oriented, independent, and demanding. With higher educational levels and the influence of democratization from the West, the younger generation has different expectations concerning their leaders. Paternalistic leadership may not be the only proper approach in the Chinese context, especially for the younger generation, as some researchers have suggested (Ramachandran \& Krishnan, 2009; Wang et al., 2005). In particular, transformational leadership appears to be an appropriate leadership style in this context for the younger generation. More generally, the findings of this study also support the use of contingency leadership because the effectiveness of leadership varies depending on individual values.

\section{Practical Implications}

Modern business management has benefited from Western managerial approaches, which emphasize scientific management, effectiveness, standardization and excellence. These Western 
management theories also have an important influence on the management philosophies of Chinese leaders, helping them to reduce costs, improve quality and customer service, strengthen R\&D, and gain competitive advantages over their competitors. Chinese leaders may consider management education and training as a vital means of developing their leadership and management skills. Actually, many Western management theories such as the concept of transformational leadership were introduced into China in the early 1990s, mainly through MBA education. The business schools in China have used original or translated teaching materials from abroad, and the business cases used are mostly from North America (Zhang et al., 2008). Although Western management practices have relevance in China, there is a gap between these practices and those in Chinese companies (Gallo, 2008). China has unique requirements compared to what is needed in the West. One would fail in China if he or she only followed all Western practices. Given the strong traditional values such as Confucianism, Chinese leaders must engage in behaviors and display values and attitudes that build and maintain harmony within the organization. Leaders in China must make hierarchical and authority structures clear to employees, understand the social rules for compliance, maintain proper relationships with others, and reciprocally generate the very conditions for a naturally harmonious social system in an organization. This effort probably is the essential task of a Chinese leader.

Consequently, Western strategic thinking must be modified and integrated with Chinese practices to be useful in China. On one hand, it is impossible to rely only on relationships to run a company; standard operating procedures also are necessary. On the other hand, it also is not possible to run Chinese companies solely on the basis of Western management practices that focus on efficiency and standard operating procedures, ignoring some traditional Chinese norms such as face saving. Furthermore, relationship considerations, together with moral persuasion, 
can supersede rules and regulations in Chinese management in many cases. Both the Chinese culture and Western management practices have merit and the combination of both allows one to manage a company effectively and efficiently in China. Foreign leaders should be more understanding of Chinese ways and Chinese leaders should be more open to using Western leadership development practices.

It has been noted that China is changing rapidly. The country is rapidly moving from a primarily agricultural nation to one of industrialization. It has moved from a planned economy to a market economy. Younger people in China lean more towards Western approaches than do older people. Obviously, most of the leaders in China are older than the average employee and most of them embrace the traditional Chinese approaches to leadership. As the younger generation increasingly becomes involved in the majority of business operations, one can predict that the business climate will become more Westernized. In addition, as China becomes integrated more into the global economy, its companies will begin to behave more like those that have been dominant in the West. Therefore, it is expected that successful Chinese leaders will continue to blend Western practices with the traditional Chinese thinking, although the trend will likely be in the direction of the more mature Western companies. From this perspective, we can see that transformational leadership fits better for the younger generation in China as a social trend, however, one should not expect the same transformational leadership that works in Western companies to work equally well in Chinese companies. Traditional Chinese cultural elements, such as the hierarchical relationship and harmony, still play a critical role in the leadership process. 
Contributions and Limitations of the Study

The major contribution of this work is that the study examined how the relationships between leadership style and effectiveness changed from an older generation to a younger generation as China develops. A model was developed suggesting that the best leader in China should integrate the key leadership concepts and practices from the West with traditional Chinese wisdom and classical Chinese leadership. An underlying assumption about the role of trust, harmony, and generation toward the leadership process also was examined. The results suggest that trust in the leader and harmonious relationships in an organization are key mechanisms for explaining effective leadership in Chinese organizations regardless of whether paternalistic or transformational leadership is used. What's more, as the younger generation is becoming the dominant workforce, a successful leader in China should use Western practices and integrate them to fit in Chinese organizations in a way that also acknowledges Chinese traditions.

Another contribution of this study is that some new measurement constructs were developed. I revised paternalistic leadership so that all three dimensions are aligned together allowing for its examination as a single construct. In addition, a new instrument for harmony was developed based on the understanding of Confucianism. This study provided tools for further understanding of Chinese leadership.

Results of this study provide several significant insights and implications for organizations to use in the leadership process; however, it is not free from limitations. Although the study was conducted in divergent contexts (e.g., private companies, state-owned companies, companies in different industries), leadership and its effectiveness was examined only at the dyadic level; that is, the manager was evaluated by his/her subordinates' individual opinions. This limits the ability to draw conclusions about the possible effect of leaders on outcomes at the 
level of the organization or the work unit. In addition, the employees were asked to evaluate their immediate supervisors, but some of the immediate supervisors are the owners of the family business companies while others are team leaders and department managers. The levels of the supervisors differ because some were from middle management, whereas others were from first line managers. As the responsibilities and requirements for managers in different levels are different, the effectiveness of leaders from upper-level and lower-level also are different. Therefore, taking into account the full complexity of the leadership phenomenon in organizations and investigating leadership using a multilevel approach would be helpful to better understand the leadership process.

Another limitation to this study was the use of self-reported data. Although self-report data is widely used, it does limit, to some degree, the validity of this study because subjects may not respond truthfully. In addition, a chance of common method variance always arises when information for a study is gathered using self-reports (Podsakoff \& Organ, 1986). Common method variance is a threat for studies that use the same subjects for independent as well as dependent variables. Unfortunately, there is no recognized standard recommendation available for eliminating common method variance. I did, however, take steps recommended in the literature (Podsakoff, MacKenzie, Lee \& Podsakoff, 2003) to avoid this problem by including some items that are positively worded and others that are negatively worded in the self-report survey. For example, the positive item, "I feel a strong loyalty to my supervisor," and the negative item. "I have a divided sense of loyalty toward my supervisor," were both used to measure the construct of trust. Generation was measured based on age rather than perceptions. Therefore, some of the impact was reduced in analyses where generation was a moderator. In addition, as leadership effectiveness is the consequence of the leader's actions for followers and 
other organizational stakeholders (Yukl, 2006), leaders' subordinates are in the best position to assess the extent to which the leader's behavior is effective because they are most likely to observe the leader's behavior on a day-to-day basis. Subordinate perception of leadership effectiveness was a key focus of this research.

\section{Future Research}

At the outset, results of my research provide useful measurement instruments for future studies that can be used to examine additional research that includes relationships in Chinese leadership/management studies. Specifically, a natural extension of this study would be a crosscultural study since it is part of the theoretical model in this research. The impact of cultural factors was discussed in Chapters 1 and 2. Because my focus was on the Chinese context and the data were only collected in Chinese organizations, future studies may compare the impacts of leadership behaviors and factors in China versus Western culture clusters. Future research may address how cultural differences (e.g., collectivism/individualism, power distance, uncertainty avoidance, masculinity/femininity, and long-term vs. short-term orientation) will impact employee's perception of trust, harmony, and leadership effectiveness. Such research will provide a clearer picture of the contributions of the paternalistic and transformational models in cross cultural research on leadership.

A further extension of the study would be the examination of interactive effects of the three dimensions of paternalistic leadership. As each of the three dimensions captures a key aspect of paternalistic leadership, logical research questions might include the following: "Do they mutually reinforce each other in producing interactive effects that go beyond their individual main effects?" "When a leader is high or low in one dimension (e.g., benevolence), do the other two dimensions (e.g., authoritative and moral leadership) continue to impact the 
outcomes?" Can one or two dimensions compensate for the lack of another one?" "Are there any contextual factors such as the acceptance of traditional values that condition their effect on outcomes?”

In addition, use of the newly developed instruments in future research studies will aid in the establishment of their validity. A cross-cultural study as mentioned earlier will provide the opportunity to use both of the new measures for paternalistic leadership and harmony. In addition, other related leadership research may use these instruments as they are often of interest in research conducted within the Chinese context. In particular, the use of the authoritative leadership subscale, part of the revised paternalistic leadership measure, should help to redefine the paternalistic leadership construct in a way that provides greater consistency in results found for all dimensions.

As pointed out, one of the limitations of this study is the lack of ability to draw a conclusion about leadership effectiveness at the organizational level. The current focus was on the internal relationships within the organization (e.g., trust in the leader and the harmonious relationship within the organization). Future study can extend the meaning of trust and harmony to the external context. As described earlier, the Chinese are very slow to trust others. When considering the relationship between business partners, the Chinese prefer to make friends and establish trust first, then do business. Westerners want to get right down to business. The Chinese often think Westerners are too rash to move forward without having established trust first; however, Westerners might lose patience and conclude that the Chinese counterpart is not trustworthy because of the slow trust-building process. Similarly, the harmony principle also can be extended to examine the relationship with the external environment such as with business 
partners, governments, and even competitors. This research would help us to further understand whether the effectiveness of leadership differs internally vs. externally.

Finally, the mediating effects of trust and harmony as well as the moderating effect of generation in the leadership process were tested. The contribution of additional factors as mediators could be considered. Researchers suggest that unlike the West, where functional knowledge is probably only secondary in selecting a leader (Gallo, 2008), Chinese employees will judge a leader's functional knowledge as the key factor of qualification. It is important for leaders in China to demonstrate their technical know-how in order to quickly win the allegiance of their team. Therefore, a leader's competence might be a potential mediator worth investigating in future studies. Some other factors such as gender, size of the company, and the property of the company (private vs. state-owned) may be included in future study to see whether these factors provide moderating effects as well since these variables were significant as control variables. For example, women in China might be accustomed to following instructions from those in higher positions (e.g., the traditional Chinese wife follows instructions from her husband who is considered to be of higher status in the household). As a result, they are more likely to accept paternalistic leadership compared to men. Small companies appear to be a better context for paternalistic leadership than large companies also because paternalistic leadership emphasizes centralization and control. Large companies use divisional or matrix structures more, which discourage centralization and control. Whether a company is private or state-owned also may have an impact. State-owned companies are more traditional such that employees expect their leaders to provide both individual as well as family care. Therefore, it is likely that these employees will view paternalistic leaders as more effective than employees from private companies. 


\section{Summary}

In this chapter, the results of the analyses, limitations, and directions for future research are discussed. From the results of the analysis, trust in the leader and harmonious relationships within an organization were found to be critically important to ensure effective leadership in the Chinese context. Further, generation was found to moderate the relationship between leadership styles and harmony /trust. Members of the older generation with stronger Chinese traditional values embedded in their way of thinking and behaving, are more likely to accept their roles and arrangements in a hierarchically structured organization and, therefore, view paternalistic leaders more positively than the younger generation. On the contrary, members of the younger generation, who are more open and receptive to Western values, view transformational leaders more positively. The best leaders in China, be they Westerners or Chinese, should notice the changing trend in China and learn how to blend the best of Western leadership practices with traditional Chinese values. 
APPENDIX A

COVER LETTER 


\section{Informed Consent Notice}

Dear Respondents,

I am conducting a study investigating effective leadership in a Chinese context. I would like for you to fill out a short survey that should take about 20 minutes to complete.

There are cultural differences between China and the West in the way workers view their leaders, what they expect from their leaders, and what leaders can expect from their workforce. The major purpose of this study is to study leadership in China. Investigating Chinese leadership not only helps to advance our knowledge of leadership in the Chinese context but also informs the study of global leadership with implications for global organizations.

All results will be examined in the aggregate, and no respondent's name or company will be published. Every feasible effort will be made to preserve anonymity. Participation is completely voluntary and participants are required to be at least 18 years of age or older. There are no foreseeable risks, and you may decline to answer any particular question that you are uncomfortable with or feel is not appropriate. Handing the survey back to me will indicate that you have given your consent for me to use the survey data as described in this paragraph. You may keep this notice for your records.

If you have any questions, please feel free to contact the principal investigator of this study, Dr. Vicki Goodwin at the University of North Texas (UNT) Department of Management at (940)565- 4766, or Wai Kwan Lau, Ph.D student at the UNT Department of Management at (940)565-3166. This research project has been reviewed and approved by the UNT Institutional Review Board (940.565.3940). Contact the UNT IRB with any questions regarding your rights as a research subject.

Thanks a lot for your help! 
APPENDIX B

SCALES FOR EACH CONSTRUCT 
Paternalistic Leadership

Authoritative Leadership

1. My supervisor knows best what is good for me.

2. The experience and wisdom of my supervisor are the best guidelines for me.

3. My supervisor expects subordinates to comply with and follow through on directives.

4. My supervisor makes final calls on key decisions.

5. My supervisor holds employees accountable for work rules and high performance standards.

Moral Leadership (Cheng et al., 2000)

1. My supervisor set himself a good role model to follow.

2. My supervisor does not take advantage of me for personal gain.

3. My supervisor is an upright and honest person; he/she never promotes his/her private interests under the guise of serving the public.

4. My supervisor never avenges a personal wrong in the name of public interest when he/she is offended (Reversed scored).

5. When a mistake occurs, my supervisor will take responsibility.

Benevolent Leadership (Cheng et al., 2000)

1. My supervisor handles what is difficult to do or manage in everyday life for me.

2. Beyond work relations, my supervisor expresses concern about my daily life.

3. My supervisor ordinarily shows a kind concern for my comfort.

4. My supervisor takes good care of my family members as well.

5. My supervisor meets my needs according to my personal requests.

6. My supervisor will help me when I'm in an emergency.

Authoritarian Leadership (Cheng et al, 2000)

1. My supervisor asks me to obey his/her instructions completely.

2. My supervisor always behaves in a commanding fashion in front of employees.

3. My supervisor determined all decisions in the organization whether they are important or not.

4. In my supervisor's mind, the standard subordinate is an employee who obeys his commands completely.

5. We have to follow supervisor's rules to get things done. If not, he/she punishes us severely.

6. My supervisor emphasizes that our group must have the best performance of all the units in the organization. (Deleted)

Transformational Leadership (Podsakoff et al., 1990) Identify and articulate a vision

1. My supervisor has a clear understanding of where we are going.

2. My supervisor paints an interesting picture of the future for our group.

3. My supervisor is always seeking new opportunities for the organization.

4. My supervisor inspires others with his/her plans for the future. 
5. My supervisor is able to get others committed to his/her dream.

Provide an appropriate model

1. My supervisor leads by "doing", rather than simply by "telling."

2. My supervisor provides a good model for me to follow.

3. My supervisor leads by example.

Fostering the acceptance of group goals

1. My supervisor fosters collaboration among work groups.

2. My supervisor encourages employees to be "team players."

3. My supervisor gets the group to work together for the same goal.

4. My supervisor develops a team attitude and spirit among employees.

High performance expectations

1. My supervisor shows us that he/she expects a lot from us.

2. My supervisor insists on only the best performance.

3. My supervisor will not settle for second best.

Provide individualized support

1. My supervisor acts without considering my feelings (Reverse scored).

2. My supervisor shows respect for my personal feelings.

3. My supervisor behaves in a manner thoughtful of my personal needs.

4. My supervisor treats me without considering my personal feelings (Reverse Scored)

Intellectual stimulation

1. My supervisor challenges me to think about old problems in new ways.

2. My supervisor asks questions that prompt me to think.

3. My supervisor has stimulated me to rethink the way I do things.

4. My supervisor has ideas that have challenged me to reexamine some of basic assumptions about my work.

Trust (Podsakoff et al., 1990)

1. I feel quite confident that my Supervisor will always try to treat me fairly.

2. My supervisor would never try to gain an advantage by deceiving workers.

3. I have complete faith in the integrity of my supervisor.

4. I feel a strong loyalty to my supervisor.

5. I would support my supervisor in almost any emergency. (Deleted)

6. I have a divided sense of loyalty toward my supervisor. (reversed)

\section{Harmony}

1. Employees behave in accordance with predetermined positions in organization.

2. In some cases, my supervisor would sacrifice task accomplishment in order to maintain harmonious relationships with and among subordinates.

3. My supervisor has the ability to balance his desire to be lenient with the need to be harsh toward his people. 
4. In complex environment, my supervisor is effective in balancing relationship so that all parties are reasonably satisfied with the result.

5. My supervisor values personal relationships

$\underline{\text { Generation }}$

Generation is a categorical variable having two categories (Generation X and Generation Y).

Generation X refers to individuals born from 1980 to 1995 . Generation Y refers to individuals born from about 1961 to 1979 .

Effectiveness (Chen \& Tjosvold, 2004)

1. My supervisor performs his/her leader roles appropriately.

2. My supervisor exercises his/her responsibilities well as a leader. (Deleted)

3. I am satisfied with my supervisor's overall effectiveness as a leader.

4. The way my supervisor manages the work inspires me to a better job performance

5. I can work effectively under the leadership of my supervisor. 


\section{REFERENCE LIST}

Adsit, D.J., London, M., Crom, S., \& Jones, D., 1997. Cross-cultural differences in upward ratings in a multinational company. International Journal of Human Resource Management. 8: $385-401$.

Aguinis, H., Petersen, S.A., \& Pierce, C.A.. 1999. Appraisal of the homogeneity of error variance assumption and alternatives to multiple regression for estimating moderating effects of categorical variables. Organizational Research Methods. 2: 315-339.

Alfredo Behrens. 2009. Culture \& Management in the Americas. Stanford, CA: Stanford University Press.

Anderson, J.C., \& Gerbing, D.W.. 1988. Structural equation modeling in practice: A review and recommended two-step approach. Psychological Bulletin. 103(3): 411-423.

Ansari, M.A., Ahmad, Z.A., \& Aafaqi, R.. 2004. Organizational leadership in the Malaysian context. In D. Tjosvold \& K. Leung (Ed.), Leading in high growth Asia: Managing relationship for teamwork and change: 109-138. Singapore: World Scientific.

Antonakis, J. \& House, R.. 2002. The full-range leadership theory: The way forward. Transformational and Charismatic Leadership: The Road Ahead. New York: Elsevier.

Arrow, K.. 1974. The Limits of Organization. New York: Norton.

Avolio, B.J., \& Bass, B.M.. 1988. Transformational leadership, charisma, and beyond. In J.G.Hunt, R.R. Baliga, H.P. Dachler, \& C.A. Schriesheim (Eds.), Emerging leadership vitas, 2949, Lexington, MA: Lexington Books.

Aycan, Z.. 2006. Paternalism: Towards conceptual refinement and operationalization. In K.S. Yang, K.K. Hwang, \& U. Kim (Eds.), Scientific advances in indigenous psychologies:

Empirical, philosophical, and cultural contributions, 445-466, London: Sage Ltd.

Aycan,Z., Kanungo, R.N., Mendonca, M., Yu, K., Deller, J., Stahl, G.. 2000. Impact of culture on human resource management practices: A 10-country comparison, Applied Psychology: An International Review. 49: 192-221.

Bass, B.M.. 1985. Leadership and Performance Beyond Expectations. New York: The Free Press.

Bass, B.M.. 1998. Transformational Leadership: Industrial, Military, and Educational Impact. Mahwah, NJ: Lawrence Erlbaum Associates.

Bass, B.M., \& Avolio, B.J.. 1993. Transformational leadership and organizational structure. International Journal of Public Administration Quarterly. 17: 112-121. 
Bass, B.M., \& Avolio, B.J.. 1994. Improving Organizational Effectiveness through Transformational Leadership. California: SAGE Publications, Inc.

Bass, B.M., \& Steidlmeier, P.. 1999. Ethics, character, and authentic transformational leadership behavior. Leadership Quarterly. 10(2): 134-143.

Bennis, W., \& Nanus, B.. 1985. Leaders: The Strategies for Taking Charge. New York: Harper \& Row.

Bernardin, H.J., Kiatt, L.A.. 1985. Managerial appraisal systems: Has practice caught up to the state of the art? Personnel Administrator. 30: 79-86.

Boal, K.B., \& Bryson, J.M,. 1988. Charismatic leadership: A phenomenological and structural approach. In J.G. Hunt, B.R., Baliga, H.P. Dachler, \& C.A. Schriesheim (Eds.), Emerging Leadership Vista (pp. 5-28). Lexington, MA: Lexington Books.

Bond, M.H., \& Hwang, K.K.. 1986. The social psychology of the Chinese people, In M.H. Bond (Eds.), The Psychology of the Chinese People (pp. 213-266). New York: Oxford University Press.

Bono, J.E., \& Judge, T.A.. 2003. Self-concordance at work: Toward understanding the motivational effects of transformational leaders. Academy of Management Journal. October: 554-571.

Bracken, B.A., Barona, A.. 1991. State of the art procedures for translating, validating and using psychoeducational tests in cross cultures assessment. School Psychology International. 12(1-2): 119-132.

Brislin, R.W.. 1970. Back-translation for cross-cultural research. Journal of Cross-Cultural Psychology. 1(3): 185-216.

Bruke, C.S., Stagl, K.C., Klein, C., Goodwin, G.F., Salas, E., \& Halpin, S.M.. 2006. What type of leadership behaviors are functional in teams? A meta-analysis. Leadership Quarterly. 17: 288307.

Bryant, S.. 2003. The role of transformational and transactional leadership in creating, sharing and exploiting organizational knowledge. The Journal of Leadership and Organizational Studies. 9(4): 32-44.

Bycio,P., Hackett, R.D., \& Allen, J.S.. 1995. Further assessment of Bass's (1985) conceptualization of transactional and transformational leadership. Journal of Applied Psychology. 80(4): 468-478.

Burns, J.M.. 1978. Leadership. New York: Harper \& Row. 
Casimir, G., Waldman, D.A., Bartram, T., \& Yang, S.. 2006. Trust and the relationship between leadership and follower performance: Opening the black box in Australia and China. Journal of Leadership and Organizational Studies. 12(3): 68-84.

Chen, X.P., Eberly, M.B., Chiang, T.J., Farh, J.L., \& Cheng, B.S.. 2011. Affective trust in Chinese leaders: Linking paternalistic leadership to employee performance. Journal of Management. first published on June 10, 2011 as doi:10.1177/0149206311410604

Chen, X.P. \& Farh, J.L. 1999. The effectiveness of transactional and transformational leader behaviors in Chinese organizations: Evidence from Taiwan. The Annual National Meeting of Academy of Management, Chicago, August, 1999.

Chen, Y.F., \& Tjosvold, D.. 2005. Cross-cultural leadership: Goal interdependence and leadermember relations in foreign ventures in China. Journal of International Management. 11: 417439.

Cheng, B.S.. 1995. Paternalistic authority and leadership: A case study of Taiwanese CEO, Bulletin of the Institute of Ethnology Academic Sinica. 79:119-173.

Cheng, B.S., Chou, L.F., \& Farh, J.L.. 2000. A triad model of paternalistic leadership: The constructs and measurement. Indigenous Psychological Research in Chinese Societies. 14: 3-64.

Cheng, B.S., Chou, L.F., Wu, T.Y., Huang, M.P., \& Farh, J.L.. 2004. Paternalistic leadership and subordinate responses: Establishing a leadership model in Chinese organizations. Asian Journal of Social Psychology. 7:89-117.

Cheng, B.S., Huang, M.P., \& Chou, L.F.. 2002a. Paternalistic leadership and its effectiveness: Evidence from Chinese organizational teams. Journal of Psychology in Chinese Societies. 3(1): 85-112.

Cheng, B.S., Shieh, P.Y., \& Chou, L.F.. 2002b. The principal's leadership, leader-member exchange quality, and the teacher's extra-role behavior: The effects of transformational and paternalistic leadership. Indigenous Psychological research in Chinese Societies. 17: 105-161.

Child, J.. 2001. Trust-The fundamental bond in global collaboration. Organizational Dynamics. 29(4): 274-288.

Chiu, C., \& Yang, C.F.. 1987. Chinese subjects' dilemmas: Humility and cognitive laziness as problems in using rating scales. Bulletin of the Hong Kong Psychological Society. 18: 39-50.

Chou, L.F, Cheng, B.S., \& Jen, C,K.. 2005. The contingent model of paternalistic leadership: subordinate dependence and leader competence. Paper presented at the meeting of the Academy of Management, Honolulu, Hawaii.

Churchill, G.A., Jr., 1979. A Paradigm for developing better measures of marketing constructs. Journal of Marketing Research. 16(February): 64-73. 
Connell, J., Ferres, N., \& Travaglione, T.. 2003. Charismatic leadership and follower effects. Journal of Personality and Social Psychology. 84(1): 46-59.

Currall, S.C, \& Judge, T.A.. 1995. Measuring trust between organizational boundary role persons. Organizational behavior and Human Decision Processes. 64: 151-170.

Dembo, M.. 2000. Reaching Generation Y. Public Relations Tactics, 7: 20-25.

Den Hartog, D. N., House, R. J., Hanges, P. J., Ruiz-Quintanilla, S. A.. 1999. Culture specific and cross-culturally generalizable implicit leadership theories: Are attributes of charismatic/transformational leadership universally endorsed? Leadership Quarterly. 10(2): 219-256.

Den Hartog, D.N., \& Dickson, M.W.. 1999, Leadership and Culture. In J. Antonakis, A.T. Cianciolo, \& R. J. Sternberg (Eds.), The nature of leadership, Thousand Oaks, CA: Sage.

Dirks, K.T., \& Ferrin, D.L.. 2002. Trust in leadership: Meta-analytic findings and implications for research and practice. Journal of applied Psychology. 87(4): 611-628.

Dou, W., Wang, G., \& Zhou, N.. 2006. Generational and regional differences in media consumption pattern of Chinese Generation X consumer. Journal of Advertising. 35: 101-110.

Dvir, T., Eden, D., Avolio, B.J., \& Shamir, B., 2002. Impact of transformational leadership on follower development and performance: A field experiment. Academy of Management Journal. 45(4): 735-744.

Elegant, S.. 2007. China's me generation. Time. 170: 32-37.

Ellen, N., Kerwin, N., \& Kerwin, K.. 1999. Generation Y. Business Week. 3619: 80-86.

England, G.W., \& Lee, R.. 1974. The relationship between managerial values and managerial success in the United States, Japan, India and Australia. Journal of Applied Psychology. 59(4): 411-419.

Ergeneli, A., Gohar, R., \& Temirbekova, Z.. 2007. Transformational leadership: Its relationship to culture value dimensions. International Journal of Intercultural Relations. 31: 703-724.

Farh, J.L., \& Cheng, B.S.. 2000. A cultural analysis of paternalistic leadership in Chinese organizations. In J.T .Li, A.S. Tsui, \& E. Weldon (Eds.), Management and organizations in the Chinese context: 84-127. London: Macmillan.

Farh, J.L., Cheng, B.S., Chou, L.F., \& Chu, X.P.. 2006. Authority and benevolence: Employees' responses to paternalistic leadership in china. In A.S. Tsui, Y. Bian, \& L. Cheng (Eds.), China's domestic priate firms: Multidisciplinary perspectives on management and performance: 230-260. New York: Sharpe. 
Ferrin, D.L., Dirks, K.T., \& Shah, P.P.. 2003. Many routes toward trust: A social network analysis of the determinants of interpersonal trust. Academy of Management Best Conference Paper (OB.C1-C6).

Frazier, P.A., Tix, A.P., \& Barron, K.E.. 2004. Testing moderator and mediator effects in counseling psychology. Journal of Counseling Psychology. 51(1): 115-134.

Fukuyama, F.. 1995. Trust: The Social Virtues and the Creation of Prosperity. Free Press: New York.

Gallo, F.T.. 2008. Business Leadership in China. River Street, Hoboken, NJ.

Gelfand, M.J., Erez, M., \& Aycan, Z.. 2007. Cross-cultural organizational behavior. Annual Review of Psychology. 58: 479-514.

Gillespie, N.A., \& Mann, L.. 2004. Transformational leadership and shared values: The building blocks of trust. Journal of Managerial Psychology. 19: 588-607.

Garrott, J.R.. 1995. Chinese cultural values: New angles, added insight. International Journal of Intercultural Relations. 19(2): 211-225.

Gurtman, M.B.. 1992. Trust, distrust, and interpersonal problems: A circumplex analysis, Journal of Personality and Social Psychology. 8: 155-174.

Hall, A.T., Blass, F.R., Ferris, G.R., \& Massengale, R.. 2004. Leader reputation and accountability in organizations: Implications for dysfunctional leader behavior. Leadership quarterly. 15: 515-536.

Hair, J.F. Jr., Anderson, R.E., Tatham, R.L., \& Black, W.C.. 1998. Multivariate Data Analysis, (5thEdition). Upper Saddle River, NJ: Prentice Hall.

Hegarty, W.H.. 1974. Using subordinate ratings to elicit behavioral changes in supervisors. Journal of Applied Psychology. 59: 764-766.

Henson, R.K., Kogan, L.R., \& Vacha-Haase, T.. 2001. A reliability generalization study of the teacher efficacy scale and related instruments. Educational and Psychological Measurement. 61: 404-420.

Hersey, P., \& Blanchard, K.H.. 1996. The Management of Organizational Behavior (7 ed.), Englewood Cliffs, NJ: Prentice-Hall.

Hofstede, G.. 1980a. Culture's Consequences: International Differences in Work-related Values (Abridged ed.). Newbury Park, CA: Sage. 
Hofstede G.. 1980b. Motivation, leadership and organization: Do American theories apply abroad? Organizational Dynamics, Summer: 42-63.

Hofstede, G.. 1991. Culture and Organizations: Software of the Mind. London: McGraw-Hill Book Company (Ltd.)

Hofstede, G.. 2001. Culture's consequences: Comparing values, behaviors, institutions, and organizations across nations. Thousand Oaks, CA: Sage.

Hogan, R., Curphy, G.J., \& Hogan,J.. 1994. What we know about leadership: Effectiveness and personality. American Psychologist. 49: 493-504.

Holtgraves, T.. 1997. Styles of language use: Individual and cultural variability in conversational indirectness. Journal of Personality and Social Psychology. 73: 624-637.

House, R.J., \& Shamir, B.. 1993. Toward the integration of transformational, charismatic, and visionary theories. In M.M. Chemers \& R. Ayman (Eds.), Leadership theory and research: Perspectives and directions (81-107). San Diego: Academic Press.

House, R.J., \& Aditya, R.N.. 1997. The social scientific study of leadership: Qua voids? Journal of Management. 23(3): 409-474.

Huang, K., \& Li, S.Y.. 2007. A netnography of beauty product enthusiasts in China:

Implications for communications planning. Journal of Advertising Research. 47(4): 485-495.

Jackofsky, EF., Slocum, J.W., Jr., \& McQuaid, S.J.. 1988. Cultural values and the CEO: Alluring companions? Academy of Management Executive. 2(1): 39-49.

Javidan, M., \& House, R.F.. 2001. Cultural acumen for the global manager: Lessons from Project BLOBE. Organizational Dynamics. 29(4): 289-305.

Javidan, M., \& Carl, D.E.. 2005. Leadership across cultures: A study of Canadian and Taiwanese executive. Management International Review. 45: 23-44.

Johnson-George, C., \& Swap, W.C.. 1982. Measurement of specific interpersonal trust: Construction and validation of a scale to assess trust inn a specific other. Journal of Personality and Social Psychology. 43(6): 1306-1317.

Judge, T.A., \& Piccolo, R.F.. 2004. Transformational and transactional leadership: A metaanalytic test of their relative validity. Journal of Applied Psychology. 89(5): 755-768.

Jung, D.I.. 2001. Transformational and transactional leadership and their effects on creativity in groups. Creativity Research Journal. 13(2): 185-195. 
Jung, D.I., Bass, B.M., \& Sosik, J.J.. 1995. Bridging leadership and culture: A theoretical consideration of transformational leadership and collectivistic cultures. Journal of Leadership Studies. 2: 3-18.

Jung, D.I., \& Avolio, B.J.. 1999. Effects of leadership style and followers' cultural orientation on performance in group and individual task conditions. Academy of Management Journal. 42(2): 208-218.

Kee, H. W., \& Knox, R.E.. 1970. Conceptual and methodological considerations in the study of trust and suspicion. Journal of Conflict Resolution. 14(3): 357-367.

Khodyakov, D.. 2007. Trust as a process: A three-dimensional approach. Sociology. 41(1): 115132.

Kouzes, J., \& Posner, B.. 1987. Overcoming the dark side of leadership: The paradox of personal dysfunction. Grand Rapids, MI: Baker Books.

Krishnan, V.R.. 2005. Transformational leadership and outcomes: Role of relationship duration. Leadership and Organization Journal. 26(5/6): 442-457.

Kuchinke, K.P.. 1999. Leadership and culture: Work-related values and leadership styles among one company's U.S. and German telecommunication employees. Human Resource Development Quarterly. 10(2): 135-154.

Lau,D.C.. 1979. Confucius: The Analects. Penguin Classics.

Lewicki, R.J., \& Bunker, B.B.. 1996. Developing and maintain trust in work relationships. In R.M. Kramer \& R.R. Tyler (Eds.), Trust in Organizations, Thousand Oaks, CA: Sage.

Lin, C.A.. 2001. Cultural values reflected in Chinese and American television advertising. Journal of Advertising. 30: 83-94.

Littrell, R. F.. 2002. Desirable Leadership Behaviors of Multi-Cultural Managers in China. The Journal of Management Development. 21(1): 5 - 74.

Lok, P. \& Crawford, J.. 2004. The effect of organizational culture and leadership style on job satisfaction and organizational commitment. The Journal of Management Development. 23(4): 321-338.

Lord, R.G., \& Maher, K.J.. 1991. Leadeership and Information Processing. London: Routledge.

Marks, M.A., Mathieu, J.E., \& Zaccaro, S.J.. 2001. A temporally based framework and taxonomy of team processes. Academy of Management Review. 26(3): 356-376.

Martinez, P.G.. 2003. Paternalism as a positive form of leader-subordinate exchange: Evidence from Mexico. Journal of Iberoamerican Academy of Management.. 1:227-242. 
Martinez, P.G. \& Dorfman, P.W.. 1998. The Mexican entrepreneur: An ethnographic study of the Mexican empresario. International Studies of Management and Organization. 28(2): 97-123.

Matzler, K., Schwarz, E., Deutinger, N, \& Harms, R.. 2008. The relationship between transformational leadership, product innovation and performance in SMEs. Journal of Small Business and Entrepreneurship. 21(2): 139-153.

McEvoy, O.M., \& Beatty, R.W.. 1989. Assessment centers and subordinate appraisals of managers: A seven-year examination of predictive validity. Personnel Psychology. 42: 37-52.

McKnight, D.H., Cummings, L.L., \& Chervany, N.L.. 1998. Initial trust formation in new organizational relationship. Academy of Management Review. 23(3): 473-490.

Michael, S.. 2005. China's Generation Y: Understanding the Future Leaders of the World's Next Superpower. Homa \& Sekey Book.

Miller, G.J.. 1992. Managerial Dilemmas: The Political Economy of Hierarchies. New York: Cambridge University Press.

Mink, O.. 1992. Creating new organizational paradigms for change. International Journal of Quality \& Reliability Management. 9: 21-23.

Myers, H.R.. 1989. Confucianism and economic development: Mainland China, Hong Kong, and Taiwan, in Conference on Confucianism and Economic Development in East Asia. Chung-Hwa Institution for Economic Research (pp. 281-304).

Niehoff, B., Enz, C., \& Grover, R.. 1990. The impact of top-management actions on employee attitudes and perceptions Group \& Organizational Studies. 15(3): 337-352.

Osland, J.S., Franco, S., \& Osland, A.. 1999. Organizational implications of Latin American culture: Lessons for the expatriate manager. Journal of Management Inquiry. 8:219-234.

Offermann, L.R., \& Hellmann, P.S.. 1997 Culture's consequences for leadership behavior: National values in action. Journal of Cross-Cultural Psychology. 28(3): 342-351.

Padavic, I., \& Earnest, W.R.. 1994. Paternalism as a component of managerial strategy. Social Science Journal. 31(4): 389-405.

Parker, R.N., \& Smith, M.D.. 1984. High correlations or multicollinearity, and what to do about either: Reply to Light. 1984 The University of North Carolina Press. 804-807.

Parsons, T., 1977. The Evolution of Societies. Englewood Cliffs, NJ: Prentice Hall.

Pearce, J.L.. 2005. Paternalism and radical organizational change. Paper presented at the meeting of the Academy of Management, Honolulu, Hawaii. 
Pellegrini, E.K., \& Scandura, T.A.. 2008. Paternalistic Leadership: A Review and Agenda for Future Research. Journal of Management. 34(3): 566-593.

Peterson, M.F., \& Hunt, J.G.. 1997. International perspectives on international leadership. Leadership Quarterly. 8(3): 203-232.

Ping, R.A. 2005. What is the average variance extracted for a latent variable interaction (or quadratic)? [on-line paper]. http://home.att.net/ rpingjr/avel.doc.

Pitt-Rivers, J.. 1993. The Spanish Bull-Right: And Kindred Activities. Anthropology Today. 9(4):11-15.

Podsakoff, P.M., Mackenzie, S.B., Moorman, R. H., \& Fetter, R.. 1990. Transformational leader behaviors and their effects on followers trust in leader, satisfaction, and organizational citizenship behaviors. Leadership Quarterly. 1(2): 107-142.

Podsakoff. P.M., \& Organ, D.W.. 1986. Self-reports in organizational research: Problems and prospects. Journal of Management. 12(2): 531-544.

Preacher, K.J., \& Hayes, A.F., 2008. Asymptotic and resampling strategies for assessing and comparing indirect effects in multiple mediator models. Behavior Research Methods. 40(3): 879-891.

Rafferty, A.E., \& Griffin, M.A.. 2004. Dimensions of transformational leadership Conceptual and empirical extensions. Leadership Quarterly. 15(3): 329-354.

Ramachandran, S., \& Krishnan, V.R.. 2009. Effects of transformational leadership on followers' affective and normative commitment: Culture as moderator. Great Lakes Herald. 3(1): 23-38.

Redding, S.G.. 1990. The Spirit of Chinese Capitalism. Berlin: Walter de Gruyter.

Rempel, J.K., Holmes, J.G., \& Zanna, M.P.. 1985. Trust in close relationships. Journal of Personality and Social Psychology. 49: 95-112.

Rich, G.. 1997. The sales manager as a role model: Effects on trust, job satisfaction, and performance of sales people. Journal of Academic Marketing Science. 25: 319-328.

Robbins, S.P., \& Judge, T.A.. 2007. Organizational Behavior. Upper Saddle River, New Jersey.

Robinson, S.L.. 1996. Trust and breach of the psychological contract. Administrative Science Quarterly. 41: 574-599.

Rogler, L.H.. 2002. Historical generations and psychology: The case of the great depression and World War II. American Psychologist. 57(12):1013-1023. 
Rousseau, D.M., Sitkin, S.B., Burt, r.S., \& Camerer, C.. 1998. Not so different after all: A crossdiscipline view of trust. Academy of Management Review. 23: 393-404.

Rotter, J.B.. 1967. A new scale for measurement of interpersonal trust. Journal of Personality, 35: 651-665.

Seymour, S.C., 1999. Women, family, and child care in India: A world in transition. Cambridge, England: Cambridge University Press.

Shin, S.J., \& Zhou, J.. 2003. Transformational leadership, conservation, and creativity: Evidence from Korea. Academy of Management Journal. December: 704-713.

Sinha, J.B.P.. 1990. Work culture in Indian context. New Delhi, India; SAGE.

Silin, R.F.. 1976. Leadership and Values. Cambridge, MA: Harvard University Press.

Shao, L., \& Webber, S.. 2006. A cross-cultural test of 'five-factor model of personality and transformational leadership'. Journal of Business Research. 59: 936-944.

Shapiro, D., Sheppard, B. H., \& Cheraskin, L.. 1992. Business on a handshake. Negotiation Journal. October: $365-377$.

Shaw, R.B.. 1997. Trust in balance: Building successful organizations on results, integrity, and concern. San Francisco, CA: Jossey-Bass Publishers.

Sheppard, B.H., \& Sherman, D.M.. 1998. The grammars of trust: A model and general implications. Academy of Management Review. 23(3): 422-437.

Sorrentino, R.M., Holmes, JG., Hanna, S.E., Sharp, A.. 1995. Uncertainty orientation and trust in close relationship: Individual differences in cognitive styles. Journal of Personality and Social Psychology. 68: 314-327.

Stanat, M.. 2005. China's Generation Y: Understanding the future leaders of the world's next superpower. Homa and Sekey Books, Paramus, NJ.

Sweetland, J.. 1978. Work in America Institute studies in productivity: Highlights of the literature: managerial productivity. Scarsdale, NY: Work in America Institute.

Tichy, N.M., \& Devanna, M.A.. 1986. The transformational leader. New York: Wiley.

Triandis, H.C. 1995. Individualism and Collectivism. Boulder, CO: West View Press.

Triandis, H.C., \& Gelfand, M.. 1998. Converging measurement of horizontal and vertical individualism and collectivism. Journal of Personality and Social Psychology. 74(1): 118-128.

Tsai, C.C. 1991. The sayings of Mencius: Wisdom in Chaotic Era. Asiapac Books. 
Tse, H.M., \& Mitchell, R.J.. 2010. A theoretical model of transformational leadership and knowledge creation: The role of open-mindedness norms and leader-member exchange. Journal of Management \& Organization. 16: 83-99.

Tucker, B.A., \& Russell, R.F.. 2004. The influence of the transformational leader. Journal of Leadership \& Organizational Studies. 10(4): 103-111.

Tyler, T.R.. 1994. Psychological models of the justice motive. Journal of Personality and Social Psychology. 67: 850-863.

Tyler, T.R., \& Degoey, P.. 1996. Trust in organizational authorities: The influence of motive attributions on willingness to accept decisions. In R.M, Kramer \& T.R., Tyler (Eds.,), Trust in Organizations. pp 331-357. Thousand Oaks, CA: Sage.

Uhl-Bien, M., Tierney, P., Graen, G., \&Wakabayashi, M., 1990. Company paternalism and the hidden investment process: Identification of the "right type" for line managers in leading Japanese organizations. Group and Organization Studies. 15:414-430.

Wah, S.S.. 2009. Chinese Leadership: Moving from Classical to Contemporary. Marshall Cavendish Editions.

Waldman, D.A., \& Spangler, W.D.. 1989. Putting together the pieces: A closer look at the determinants of job performance. Human Performance. 2(1): 29-59.

Waldman, D.A., Bass, B.M., \& Yammarino, F.S.. 2000. Adding to contingent-reward behavior: The augmenting effect of charismatic leadership. Group and Organizational Studies. 15(4): 381394.

Walumbwa, F.O., \& Lawler, J.J.. 2003. building effective organizations: Transformational leadership, collectivist orientation, work-related attitudes and withdrawal behaviors in three emerging economies. International Journal of Human Resource Management. 14: 1083-1101.

Wang, H., Law, K., Hackett, R., Wang, D., \& Chen, Z.X.. 2005. Leader-member exchanges as a mediator of the relationship between transformational leadership and followers' performance and organizational citizenship behavior. Academy of Management Journal. 48: 420-432.

Weber, M.. 1947. The theory of social and economic organization, A.M. Henderson \& T. Parsons, Trans.. New York; Free Press.

Weber, M.. 1978. The types of legitimate domination. In G. Roth \& C. Wittich (Eds.), Economy and society. 3: 212-216, New York: Bedminister.

Westwood, R.I.. 1997. Harmony and patriarchy: The cultural basis for 'paternalistic headship' among the overseas Chinese. Organization Studies. 18: 445-480. 
Whitener, E.M., Brodt, S.E., Korsgaard, M.A., \& Werner, J.M.. 1998. Managers as initiators of trust: An exchange relationship framework for understanding managerial trustworthy behavior. Academy of Management Review. 23(3): 513-530.

Williams, M.. 2001. In whom we trust group membership as an affective context for trust development. Academy of Management Review. 26(3): 377-396.

Wofford, J.C., \& Goodwin, V.L.. 1994. A cognitive interpretation of transactional and transformational leadership theories. Leadership Quarterly. 5:161-186.

Wolburg, J.M., \& Pokrywczynski, J.. 2001. A psychographic analysis of Generation Y college students. Journal of Advertising Research. 41: 33-52.

Yang, K.S..1998. Chinese responses to modernization: A psychological analysis. Asian Journal of Social Psychology. 1: 75-97.

Yang, X.H., Peng, Y.Q., \& Lee, Y.T.. 2008. The Confucian and Mencian philosophy of benevolent leaderhip. Inn C.C. Chen \& Y.T.Lee (Eds.) Leadership and management in China: Philosophies, theories, and practice (pp. 31-50). New York: Cambridge University Press.

Yin, R.. 1989. Case Study Research: Design and Methods (Rev. ed.). Newbury Park, CA: Sage Publishing.

Yu, H., Leithwooe, K., \& Jantzi, D.. 2002. The effects of transformational leadership on teachers' commitment to change in Hong Kong. Journal of Educational Administration. 40(4): 368-389.

Yukl, G.A.. 1989. Managerial leadership: A review of theory and research. Yearly Review of Management. 15: 251-289.

Yukl, G.A.. 2006. Leadership in Organizations. $6^{\text {th }}$ Ed., New Jersey: Pearson Education, Inc.

Zaccaro, S.J., \& banks, D.J.. 2001. Leadership, vision, and organizational effectiveness. In S.J.Zaccaro \& RJ.Klimoski (Eds.), The nature of organizational leadership (pp.181-218). San Francisco: Jossey-Bass.

Zaidman, N., \& Brock, D.M.. 2009. Knowledge transfer within multinationals and their foreign subsidiaries: A culture-context approach. Group \& Organization Management. 34: 292-329.

Zeffane, R., \& Connell, J.. 2003. Trust and HRM in the new millennium. International Journal of Human Resource Management. 14(2): 1-9.

Zend, D.E.. 1997. The Leadership Triad: Knowledge, Trust, and Power. New York: Oxford Press. 
Zhang, Z.X., Chen, C.C., Liu, L.A., \& Liu, X.F.. 2008. Chinese traditions and Western theories: Influences on business leaders in China. In C.C. Chen \& Y.T. Lee (Eds.), Leadership and Management in China. Cambridge University Press.

Zhang, J., \& Sharitt, S.. 2003. Cultural values in advertisement to the Chinese X-generation. Journal of Advertising. 32: 23-33.

Zhao, E.D., \& Liu, L.W.. 2006. Generational difference and employee retaining-taking construction enterprises in China Electric Power University as examples, Proceedings of CRIOM 2006 International Research Symposium on Advancement of Construction Management and Real Estate. pp. 170-176. 